\title{
ON A DIFFUSIVE VERSION OF THE LIFSCHITZ-SLYOZOV-WAGNER EQUATION
}

\author{
JOSEPH G. CONLON
}

\begin{abstract}
This paper is concerned with the Becker-Döring (BD) system of equations and their relationship to the Lifschitz-Slyozov-Wagner (LSW) equations. A diffusive version of the LSW equations is derived from the BD equations. Existence and uniqueness theorems for this diffusive LSW system are proved. The major part of the paper is taken up with proving that solutions of the diffusive LSW system converge in the zero diffusion limit to solutions of the classical LSW system. In particular, it is shown that the rate of coarsening for the diffusive system converges to the rate of coarsening for the classical system.
\end{abstract}

\section{INTRODUCTION.}

In this paper we shall be concerned with the Becker-Döring (BD) system of equations 2 and their relationship to the Lifschitz-Slyozov-Wagner (LSW) equations 11, 22. The BD equations describe the time evolution for a mean field model of a population of particle clusters. Particle clusters are characterized by their volume $\ell$ which may take integer values $\ell=1,2 \ldots$. The monomers of volume $\ell=1$ play a distinctive role since they mediate interactions between particle clusters of different volumes. In particular, a cluster with volume $\ell$ can become a cluster of volume $\ell+1$ by addition of a monomer, or become a cluster of volume $\ell-1$ by evaporation of a monomer. Finally, conservation of mass imposes a constraint which is non-local in $\ell$.

The BD equations are determined by the rates at which a cluster of volume $\ell$ becomes a cluster of volume $\ell+1$ or $\ell-1$. If $c_{1}(t)$ is the monomer density at time $t$, then a cluster of volume $\ell$ becomes a cluster of volume $\ell+1$ at rate $a_{\ell} c_{1}(t)$, and a cluster of volume $\ell$ evaporates a monomer at rate $b_{\ell}$ to become a cluster of volume $\ell-1$. The net effect of these two processes yields a flux $J_{\ell}$ of $\ell$-clusters to $\ell+1$-clusters which depends on the density $c_{\ell}(t)$ of $\ell$-clusters and $c_{\ell+1}(t)$ of $\ell+1$-clusters, as well as the monomer density $c_{1}(t)$. In the BD model it is given by the formula $J_{\ell}=a_{\ell} c_{1} c_{\ell}-b_{\ell+1} c_{\ell+1}$. The $\mathrm{BD}$ equations for the time evolution of the system are therefore given by

$$
\begin{aligned}
\frac{d c_{\ell}}{d t} & =J_{\ell-1}-J_{\ell}, \quad \ell \geq 2, \\
\sum_{\ell=1}^{\infty} \ell c_{\ell}(t) & =\rho .
\end{aligned}
$$

1991 Mathematics Subject Classification. 35F05, 82C70, 82C26.

Key words and phrases. nonlinear pde, coarsening. 
Global existence and uniqueness theorems for the BD system (1.1), (1.2) were proven in the seminal paper of Ball et al [1] under fairly mild assumptions on the rates $a_{\ell}, b_{\ell}, \ell=1,2 \ldots$, the main one being that $a_{\ell}$ should grow at a sub-linear rate as $\ell \rightarrow \infty$.

It is easy to see by solving $J_{\ell}=0, \ell \geq 1$, that (1.1), (1.2) has a family of equilibrium solutions parametrized by the monomer density $c_{1}$. In order to classify the equilibrium solutions one needs detailed knowledge of the asymptotic behavior of the rates $a_{\ell}, b_{\ell}$ as $\ell \rightarrow \infty$. In this paper we shall assume that $a_{\ell}, b_{\ell}$ are given by the formulae

$$
a_{\ell}=a_{1} \ell^{1 / 3}, \quad a_{1}>0 ; \quad b_{\ell}=a_{\ell}\left(z_{s}+q / \ell^{1 / 3}\right), \quad z_{s}, q>0 .
$$

These values for $a_{\ell}, b_{\ell}$ may be obtained by deriving the BD equations as mean field equations for a 3 dimensional microscopic system [20].

With $a_{\ell}, b_{\ell}$ given by (1.3), one sees that for any $c_{1}$ satisfying $0<c_{1} \leq z_{s}$ there is an equilibrium solution $c_{\ell}=Q_{\ell} c_{1}^{\ell}, \ell=1,2 \ldots$. For large $\ell$ the coefficient $Q_{\ell}$ has the asymptotic behavior

$$
Q_{\ell} \sim z_{s}^{-(\ell-1)} \frac{1}{\ell^{1 / 3}} \exp \left[-\frac{3 q}{2 z_{s}} \ell^{2 / 3}\right], \quad \ell \rightarrow \infty .
$$

It follows from (1.4) that there is an equilibrium solution corresponding to $c_{1}=z_{s}$. We shall denote its density (1.2) by $\rho_{\text {crit }}$. It was shown in [1] that if $\rho \leq \rho_{\text {crit }}$, then the solution of (1.1), (1.2) converges strongly at large time to the corresponding equilibrium solution in the sense that

$$
\lim _{t \rightarrow \infty} \sum_{\ell=1}^{\infty} \ell\left|c_{\ell}(t)-Q_{\ell} c_{1}^{\ell}\right|=0 .
$$

If $\rho>\rho_{\text {crit }}$ then $c_{\ell}(t)$ converges weakly to the equilibrium solution with maximum density,

$$
\lim _{t \rightarrow \infty} c_{\ell}(t)=Q_{\ell} z_{s}^{\ell}, \ell \geq 1
$$

Equation (1.5) shows that for $\rho>\rho_{\text {crit }}$ particles from the excess density $\rho-\rho_{\text {crit }}$ over equilibrium concentrate at large time in clusters of increasingly large volume. This is the phenomenon of coarsening, and it is an important problem to understand the rate at which the volume of clusters from the excess density increase with time.

A mechanism for determining this was proposed by Penrose in 20, where he argued that the large time behavior of clusters from the excess density is governed by the LSW equations,

$$
\begin{aligned}
\frac{\partial c(x, t)}{\partial t} & =\frac{\partial}{\partial x}\left[\left\{1-\left(\frac{x}{L(t)}\right)^{1 / 3}\right\} c(x, t)\right], \quad x>0, \\
\int_{0}^{\infty} x c(x, t) d x & =\rho-\rho_{\text {crit }} .
\end{aligned}
$$

The parameter $L(t)$ in (1.6), which turns out to be a measure of the average cluster volume, is uniquely determined by the conservation law (1.7). A mathematically rigorous relationship between solutions of the BD system (1.1), (1.2) and LSW system (1.6), (1.7) was established in [10] for the case $z_{s}=0$ when $\rho_{\text {crit }}=0$, and in 13 for the case $z_{s}>0$ when $\rho_{\text {crit }}>0$. Much still remains to be done however, in order to understand this in the precise way envisioned by Penrose [20]. 
In this paper we take a rather different approach from [10, 13, in attempting to establish a relationship between solutions of the BD and LSW systems. Following [21, we first observe that the flux $J_{\ell}$ can be written as

$$
J_{\ell}=\left[b_{\ell} c_{\ell}-b_{\ell+1} c_{\ell+1}\right]-a_{1} q\left[1-\{\ell / L(t)\}^{1 / 3}\right] c_{\ell},
$$

where $L(t)$ is given by the formula

$$
c_{1}(t)=z_{s}+q / L(t)^{1 / 3} .
$$

Observe from (1.5) that the function $L(t)$ in (1.8) satisfies $L(t) \rightarrow \infty$ as $t \rightarrow \infty$. Denoting by $D$ the forward difference operator and $D^{*}$ its adjoint, then (1.1) is given by

$$
\frac{\partial c(\ell, t)}{\partial t}=-D^{*} D\left[b_{\ell} c(\ell, t)\right]-a_{1} q D^{*}\left[\left(1-\{\ell / L(t)\}^{1 / 3}\right) c(\ell, t)\right], \quad \ell \geq 2,
$$

where $c(\ell, t)=c_{\ell}(t)$ in (1.1). Evidently if we drop the first term on the RHS of (1.9) we obtain a discrete version of (1.6).

We still need to account for the discrepancy in the conservation laws (1.2) for the BD system and (1.7) for the LSW system. Now from (1.5) the bulk of the equilibrium density $\rho_{\text {crit }}$ is concentrated in clusters of volume $O(1)$ as $t \rightarrow \infty$. Further, by choosing $\ell^{*}$ large enough we may make $c\left(\ell^{*}, t\right)$ arbitrarily small for all large $t$, and the density concentrated in clusters with volume larger than $\ell^{*}$ arbitrarily close to $\rho-\rho_{\text {crit }}$. It may seem reasonable therefore, that in order to understand the large time behavior of the BD system, one is justified in setting $c\left(\ell^{*}, t\right)=0, t \geq T$, for some large but fixed $\ell^{*}$. This assumption is evidently unphysical since it cuts off interaction between clusters of volume less than $\ell^{*}$ and clusters of volume greater than $\ell^{*}$. One consequence of it is that the resulting conservation of mass for clusters of size greater than $\ell^{*}$ implies $c_{1}(t)>z_{s}$ for $t>T$. There is so far no rigorous proof that solutions of the BD equations with $\rho>\rho_{\text {crit }}$ satisfy $c_{1}(t)>z_{s}, t>T$, for sufficiently large $T$. A proof of it would seem to be necessary in order to rigorously justify the relationship between the BD and LSW systems. In this connection one should also note that in 13 it is necessary to make an assumption -equation (1.33) of the paper-in order to prove that a scaling limit of the BD system is a solution of the LSW system. Equation (1.33) is however not a boundary condition, but essentially an upper bound assumption on the rate of coarsening for the BD model. Some interesting heuristic explanations [5 have recently been given to describe the transition, from the relaxation to equilibrium phase of the $\mathrm{BD}$ time evolution, to the coarsening phase governed by the LSW equations. These may be helpful towards constructing a fully rigorous theory.

We shall assume now that there is a time $T$ such that for $t \geq T$, the interaction between clusters of large volume and clusters of $O(1)$ volume is negligible, and even make the significantly stronger assumption that there exists $\ell^{*} \geq 1$, such that $c\left(\ell^{*}, t\right)=0, t \geq T$. We may therefore study the large time evolution of the cluster density for particles from the excess density by solving the initial value problem for (1.9), subject to conservation of mass for particles with volume larger than $\ell^{*}$. Without loss of generality we may normalize $\ell^{*}=1, T=0$, whence the problem becomes solving (1.9) subject to the Dirichlet boundary condition $c(1, t)=0, t \geq 0$, given initial condition $c_{\ell}(0)=\gamma_{\ell}, \ell \geq 1$, and conservation law

$$
\sum_{\ell=1}^{\infty} \ell c(\ell, t)=\rho-\rho_{\text {crit }}
$$


In $\S 2$ we prove global existence and uniqueness of a solution to this problem (Theorem 2.2), and obtain some properties of it.

Next we consider a continuous version of (1.9). Setting $x \sim \ell$ and replacing finite differences in (1.9) by derivatives, we obtain the equation

$$
\frac{\partial c(x, t)}{\partial t}=\frac{\partial^{2}}{\partial x^{2}}\left[\varepsilon(1+x / \varepsilon)^{1 / 3} c(x, t)\right]+\frac{\partial}{\partial x}\left[\left\{1-\left(\frac{x}{L(t)}\right)^{1 / 3}\right\} c(x, t)\right], \quad x>0,
$$

with the parameter $\varepsilon=1$ in (1.11). In (1.11) we have replaced the coefficient $b_{\ell}=a_{1}\left(q+z_{s} l^{1 / 3}\right)$ of (1.9) by the diffusion coefficient $(1+x)^{1 / 3}$. This is simply for convenience, since the only properties of the diffusion coefficient in (1.11) which we will use are that it behaves like $x^{1 / 3}$ for large $x$, and is bounded away from 0 for small $x$. An equation similar to (1.11) was obtained in 21. In $\S 3$ we prove global existence and uniqueness of solutions to (1.11) subject to the constraint (1.7) and the Dirichlet boundary condition $c(0, t)=0, t>0$, provided the nonnegative initial data $c(x, 0), x>0$, satisfies the integrability property,

$$
\int_{0}^{\infty}(1+x) c(x, 0) d x<\infty .
$$

This is the content of Theorem 3.1. In 21] an analogous local existence and uniqueness theorem is proved (Theorem 6 of [21]), for which one must also assume the point-wise estimate $\sup _{x>0} c(x, 0) /(1+x)^{2 / 3}<\infty$ on the initial data.

There are several existence and uniqueness theorems in the literature for the LSW system (1.6), (1.7) and its generalizations. Collet and Gordon 3 , have proved existence and uniqueness for a system with globally Lipschitz coefficients, under the condition that the initial data $c(\cdot, 0)$ is integrable and has finite first moment. The corresponding result for the LSW system-in which the coefficients are not globally Lipschitz- was proved by Laurençot [9]. Niethammer and Pego [15] proved existence and uniqueness for the LSW system under the condition that the initial data $c(\cdot, 0)$ is a Borel measure with compact support. In [16] they were able to extend their method to the case when the initial data $c(\cdot, 0)$ is only assumed to be a Borel measure with finite first moment.

In $\S 4, \S 5$ we shall show that the solution of the diffusive LSW system (1.7), (1.11) with given initial data, converges as $\varepsilon \rightarrow 0$ on any finite time interval to the solution of the classical LSW system with the same initial data. One hopes that these results will shed some light on the problem of understanding the mechanism of coarsening for the BD and other models of Ostwald ripening. It has been argued in the physics literature [12] that in models of Ostwald ripening a diffusive LSW equation occurs. Although the effect of the diffusion term vanishes at large time it acts as a selection principle, so that the large time behavior of the model is governed by the unique infinitely differentiable self-similar solution of the LSW model.

The fact that the effect of the diffusion term is expected to vanish at large time is closely related to the dilation invariance property of the LSW system. Thus if $c(x, t), L(t), t>0$, is a solution to (1.6), (1.7), then for any $\lambda>0$ one has that $c_{\lambda}(x, t), L_{\lambda}(t), t>0$, defined by $c_{\lambda}(x, t)=\lambda c(\lambda x, \lambda t), L_{\lambda}(t)=L(\lambda t) / \lambda, t>0$, is also a solution. It follows that one may solve the LSW system for large time iteratively by solving (1.6), (1.7) on the unit time interval $0 \leq t \leq 1$ with $L(0)=1$, and then rescaling $L(1)$ to 1 . Hence, assuming that in the iteration one always 
has for some constants $a, b$ satisfying $1<a \leq b$ the inequality $a \leq L(1) \leq b$, the function $L(t)$ increases linearly in $t$ as $t \rightarrow \infty$. This is a quantitative statement concerning the phenomenon of coarsening.

Consider now how this iteration scheme would apply to the diffusive LSW system (1.7), (1.11). Denoting the solution to (1.11) by $c_{\varepsilon}(x, t), L_{\varepsilon}(t)$, then dilation using the parameter $\lambda$ results in a function $\lambda c_{\varepsilon}(\lambda x, \lambda t)$ which is a solution to (1.7), (1.11) with $\varepsilon$ in (1.11) replaced by $\varepsilon / \lambda$. Thus in the iteration scheme described above $\varepsilon \sim 1 / L(t) \rightarrow 0$ for large time $t$, corresponding roughly to an equivalence of $\varepsilon \sim$ $1, t \rightarrow \infty$ and $\varepsilon \rightarrow 0, t \sim 1$.

We summarize our main results on convergence of solutions of the diffusive LSW system to solutions of the classical LSW system. In $\S 4$ we prove that if $c_{\varepsilon}(x, t), L_{\varepsilon}(t), t>0$, is the solution to (1.7), (1.11) with initial data satisfying (1.12) then

$$
\begin{aligned}
\lim _{\varepsilon \rightarrow 0} \int_{x}^{\infty} c_{\varepsilon}\left(x^{\prime}, t\right) d x^{\prime} & =\int_{x}^{\infty} c_{0}\left(x^{\prime}, t\right) d x^{\prime}, \quad x \geq 0, \\
\lim _{\varepsilon \rightarrow 0} L_{\varepsilon}(t) & =L_{0}(t), \quad t \geq 0,
\end{aligned}
$$

where $c_{0}(x, t), L_{0}(t), t>0$, is the corresponding solution to the classical LSW system (1.6), (1.7). This is the content of Theorem 4.1. In $\S 5$ we show that the point-wise in time coarsening rate for the diffusive LSW system converges as $\varepsilon \rightarrow 0$ to the corresponding rate for the LSW system. Specifically, let us define $\Lambda_{\varepsilon}(t), t \geq 0$, by

$$
\Lambda_{\varepsilon}(t)=\int_{0}^{\infty} x c_{\varepsilon}(x, t) d x / \int_{0}^{\infty} c_{\varepsilon}(x, t) d x, \quad t \geq 0,
$$

so $\Lambda_{\varepsilon}(t)$ is the mean cluster volume at time $t \geq 0$. Then if the initial data satisfies (1.12), the function $\Lambda_{\varepsilon}(t), t>0$, is differentiable for $\varepsilon \geq 0$ and

$$
\lim _{\varepsilon \rightarrow 0} \frac{d \Lambda_{\varepsilon}(t)}{d t}=\frac{d \Lambda_{0}(t)}{d t}, \quad t \geq 0
$$

This is the content of Theorem 5.1.

In [4] an upper bound on the time averaged coarsening rate for the LSW system is obtained by application of a rather general argument of Kohn and Otto 8. At the end of $\S 3$ we extend the method of 4 to prove an upper bound on the time averaged coarsening rate for the diffusive LSW system (1.7), (1.11) with $0<\varepsilon \leq 1$, which is uniform as $\varepsilon \rightarrow 0$. This is the content of Theorem 3.2.

\section{Existence and Uniqueness for the Discrete Case}

We begin by giving a proof of the existence and uniqueness of solutions to the Becker-Döring system (1.1), (1.2) which is somewhat different than that given in 1. We assume that the initial data $c_{\ell}(0), \ell \geq 1$, satisfies

$$
c_{\ell}(0)=\gamma_{\ell}, \ell \geq 1, \sum_{\ell=1}^{\infty} \ell \gamma_{\ell}=\rho>0
$$

where the $\gamma_{\ell}, \ell \geq 1$, are all non-negative. For any $\delta>0$ we define a space $X_{\delta}$ by $X_{\delta}=\left\{c_{1}:[0, \delta] \rightarrow \mathbf{R}: c_{1}(0)=\gamma_{1}, c_{1}\right.$ is continuous and $\left.0 \leq c_{1}(t) \leq \rho, 0 \leq t \leq \delta\right\}$. 
Now $X_{\delta}$ is a complete metric space under the uniform norm, $\|\cdot\|_{\delta}$. We define a mapping $T: X_{\delta} \rightarrow X_{\delta}$ which has the property that the solutions to the BeckerDöring system give rise to fixed points of the mapping. To do this we consider functions $v(\ell, t), \ell \geq 1, t>0$, and define $J(\ell, v, t)$ for $\ell \geq 1, t>0$, by

$$
J(\ell, v, t)=a_{\ell} c_{1}(t) v(\ell, t)-b_{\ell+1} v(\ell+1, t),
$$

where $c_{1}(t), t>0$, is a given non-negative function. Let $c(\ell, t), \ell \geq 2, t>0$, be the solution to the linear system of equations,

$$
\frac{\partial c(\ell, t)}{\partial t}=J(\ell-1, c, t)-J(\ell, c, t), \quad \ell \geq 2
$$

with initial and boundary conditions given by

$$
c(1, t)=c_{1}(t), t>0, \quad c(\ell, 0)=\gamma_{\ell}, \ell \geq 2 .
$$

By the maximum principle there is a unique positive solution to (2.3), (2.4). The mapping $T: X_{\delta} \rightarrow X_{\delta}$ is defined as follows: For $c_{1} \in X_{\delta}$ let $c(\ell, t), 0 \leq t \leq \delta$, be the corresponding solution of (2.3), (2.4). Then $T c_{1}$ is given by the formula

$$
T c_{1}(t)=\max \left\{\rho-\sum_{\ell=2}^{\infty} \ell c(\ell, t), 0\right\}, \quad 0 \leq t \leq \delta .
$$

Evidently $T c_{1} \in X_{\delta}$. We show that for $\delta$ sufficiently small $T$ is a contraction.

Lemma 2.1. There exists $\delta(\rho)>0$ depending only on $\rho$, such that for $0<\delta<\delta(\rho)$ the mapping $T$ on $X_{\delta}$ is a contraction.

Proof. We have from (2.3) that

$$
\sum_{\ell=2}^{\infty} \ell c(\ell, t)=\sum_{\ell=2}^{\infty} \ell \gamma_{\ell}+\int_{0}^{t} d s J(1, c, s)+\sum_{\ell=1}^{\infty} J(\ell, c, s) .
$$

It follows that there is a constant $K>0$, independent of $\rho, \delta$, such that

$$
K \sum_{\ell=2}^{\infty} \ell c(\ell, t) \leq \sum_{\ell=2}^{\infty} \ell \gamma_{\ell}+\delta[1+\rho]\left\|\sum_{\ell=1}^{\infty} \ell^{1 / 3} c(\ell, \cdot)\right\|_{\delta},
$$

for $0 \leq t \leq \delta$. It follows from this that for $\delta$ sufficiently small, depending only on $\rho$, there is the inequality,

$$
\left\|\sum_{\ell=1}^{\infty} \ell c(\ell, \cdot)\right\|_{\delta} \leq K_{1} \rho
$$

for some constant $K_{1}$ independent of $\rho$ and $\delta$.

Next we consider $c_{1}, c_{1}^{\prime} \in X_{\delta}$ and for $0 \leq \lambda \leq 1$, let $c_{\lambda, 1}=\lambda c_{1}+(1-\lambda) c_{1}^{\prime}$. Define $c_{\lambda}(\ell, t), \ell \geq 2,0<t \leq \delta$, to be the solution of (2.3), (2.4) with $c_{\lambda, 1}$ substituted for $c_{1}$. Putting $u_{\lambda}(\ell, t)=\partial c_{\lambda}(\ell, t) / \partial \lambda$ it follows that $u_{\lambda}$ satisfies the equation,

$\frac{\partial u_{\lambda}(\ell, t)}{\partial t}=J\left(\ell-1, u_{\lambda}, t\right)-J\left(\ell, u_{\lambda}, t\right)+\left[c_{1}(t)-c_{1}^{\prime}(t)\right]\left[a_{\ell-1} c_{\lambda}(\ell-1, t)-a_{\ell} c_{\lambda}(\ell, t)\right], \ell \geq 2, t>0$,

with the initial and boundary conditions,

$$
u_{\lambda}(1, t)=c_{1}(t)-c_{1}^{\prime}(t), t>0, u_{\lambda}(\ell, 0)=0, \ell \geq 2 .
$$


Let $v_{\lambda}(\ell, t), \ell \geq 2,0 \leq t \leq \delta$, be the solution to the equation, $(2.10)$

$\frac{\partial v_{\lambda}(\ell, t)}{\partial t}=J\left(\ell-1, v_{\lambda}, t\right)-J\left(\ell, v_{\lambda}, t\right)+\left\|c_{1}-c_{1}^{\prime}\right\|_{\delta}\left[a_{\ell-1} c_{\lambda}(\ell-1, t)+a_{\ell} c_{\lambda}(\ell, t)\right], \ell \geq 2,0 \leq t \leq \delta$, with the initial and boundary conditions,

$$
v_{\lambda}(1, t)=\left\|c_{1}-c_{1}^{\prime}\right\|_{\delta}, t>0, \quad v_{\lambda}(\ell, 0)=0, \quad \ell \geq 2 .
$$

By the maximum principle $v_{\lambda}(\ell, t)$ is positive for $\ell \geq 2, t>0$, and there is the inequality

$$
\left|u_{\lambda}(\ell, t)\right| \leq v_{\lambda}(\ell, t), \quad \ell \geq 2, \quad 0 \leq t \leq \delta .
$$

From (2.10), (2.11) we have that

$$
\begin{aligned}
\sum_{\ell=2}^{\infty} \ell^{1 / 3} v_{\lambda}(\ell, t) & =\int_{0}^{t} d s\left\{2^{1 / 3} J\left(1, v_{\lambda}, s\right)+\sum_{\ell=2}^{\infty}\left[(\ell+1)^{1 / 3}-\ell^{1 / 3}\right] J\left(\ell, v_{\lambda}, s\right)\right\} \\
& +\left\|c_{1}-c_{1}^{\prime}\right\|_{\delta} \int_{0}^{t} d s\left\{2^{1 / 3} a_{1} c_{\lambda}(1, s)+\sum_{\ell=2}^{\infty}\left[(\ell+1)^{1 / 3}+\ell^{1 / 3}\right] a_{\ell} c_{\lambda}(\ell, s)\right\} .
\end{aligned}
$$

Using (2.7) and applying Gronwall's inequality in the previous equation, we see that there is a constant $K_{2}$ independent of $\rho$ and $\delta$ such that

$$
\left\|\sum_{\ell=2}^{\infty} \ell^{1 / 3} v_{\lambda}(\ell, \cdot)\right\|_{\delta} \leq K_{2} \delta \rho\left\|c_{1}-c_{1}^{\prime}\right\|_{\delta}
$$

provided $\delta$ is taken sufficiently small, depending only on $\rho$.

To see that $T$ is a contraction for small $\delta$ observe that

$$
\left|T c_{1}(t)-T c_{1}^{\prime}(t)\right| \leq\left|\sum_{\ell=2}^{\infty} \ell\left[c_{1}(\ell, t)-c_{0}(\ell, t)\right]\right| .
$$

Hence on using (2.6) we have that

$$
\left|T c_{1}(t)-T c_{1}^{\prime}(t)\right| \leq K_{3}[1+\rho] \int_{0}^{t} d s\left\{\left|c_{1}(s)-c_{1}^{\prime}(s)\right|+\sum_{\ell=2}^{\infty} \ell^{1 / 3}\left|\int_{0}^{1} u_{\lambda}(\ell, s) d \lambda\right|\right\}, 0 \leq t \leq \delta,
$$

for a constant $K_{3}$ independent of $\rho, \delta$. The result follows from the last inequality, (2.12), (2.13).

Theorem 2.1. The Becker-Döring system (1.1), (1.2) with initial condition satisfying (2.1) has a unique global in time solution.

Proof. It is evident that a solution of the Becker-Döring system (1.1), (1.2) with initial condition (2.1) gives rise to a fixed point of the mapping $T$ of (2.5). Hence by Lemma 2.1 the solution is unique. Correspondingly, suppose $c_{1}$ is a fixed point of $T$. Similarly to (2.6) we have that

$$
\begin{gathered}
\sum_{\ell=2}^{\infty} \ell[c(\ell, t)-c(\ell, t+\eta)]=-\int_{t}^{t+\eta} d s\{J(1, c, s) \\
\left.+\sum_{\ell=2}^{\infty} J(l, c, s)\right\} \geq-K \rho \int_{t}^{t+\eta} c_{1}(s) d s,
\end{gathered}
$$


for $0<\eta<\delta / 2,0<t<\delta / 2$, where $K>0$ is a constant independent of $\delta, \rho$. Since $T c_{1}=c_{1}$ it follows from (2.5) that $c_{1}$ satisfies the inequality,

$$
c_{1}(t+\eta)-c_{1}(t) \geq-K \rho \int_{t}^{t+\eta} c_{1}(s) d s .
$$

Hence if $c_{1}(0)>0$ then $c_{1}(t)>0$ for $0<t<\delta / 2$. Conversely if $c_{1}(0)=0$ there is a $t_{0} \geq 0$ such that $c_{1}(t)=0$ for $t \leq t_{0}$ and $c_{1}(t)>0$ for $t_{0}<t<\delta / 2$. Suppose $t_{0}>0$. Then (2.6) implies that

$$
\sum_{\ell=2}^{\infty} \ell c(\ell, t)<\sum_{\ell=2}^{\infty} \ell \gamma_{\ell}, \quad 0<t \leq t_{0}
$$

Since $T c_{1}=c_{1}$ we conclude from (2.5) that $c_{1}(t)>0$ for $0<t<t_{0}$, a contradiction. Thus $t_{0}=0$ whence $c_{1}(t)>0$ for $0<t<\delta / 2$ in all situations. It follows then from (2.5) that the conservation law (1.2) is satisfied. Thus $c_{1}(t), c(\ell, t), \ell \geq 2$, are a solution of the Becker-Döring system (1.1), (1.2) .

Next we establish existence and uniqueness for (1.9), (1.10) subject to the Dirichlet boundary condition $c(1, t)=0, t \geq 0$. For a given non negative function $c_{1}(t), t \geq 0$, let $c(\ell, t), \ell \geq 2, t>0$, be the solution to (2.3) subject to the initial and boundary conditions,

$$
c(1, t)=0, t \geq 0, \quad c(\ell, 0)=\gamma_{\ell}, \ell \geq 2,
$$

where the $\gamma_{\ell}$ satisfy (2.1) with $\gamma_{0}=0, \rho=1$. Normalizing $\rho-\rho_{\text {crit }}=1$ in (1.10), the function $c_{1}(t), t \geq 0$, is then to be chosen to maintain the conservation law

$$
\sum_{\ell=2}^{\infty} \ell c(\ell, t)=1 \text {. }
$$

Assuming we have a solution to (2.3), (2.14), (2.15) it follows that

$$
\begin{aligned}
0 & =\frac{\partial}{\partial t} \sum_{\ell=2}^{\infty} \ell c(\ell, t)=J(1, c, t)+\sum_{\ell=1}^{\infty} J(\ell, c, t) \\
& =c_{1}(t) \sum_{\ell=2}^{\infty} a_{\ell} c(\ell, t)-\sum_{\ell=2}^{\infty} b_{\ell} c(\ell, t)-b_{2} c(2, t) .
\end{aligned}
$$

We conclude therefore that $c_{1}(t)$ is given by the formula,

$$
c_{1}(t)=z_{s}+\left\{a_{1} q \sum_{\ell=2}^{\infty} c(\ell, t)+b_{2} c(2, t)\right\} / \sum_{\ell=2}^{\infty} a_{\ell} c(\ell, t) .
$$

Setting $t=0$ in (2.16) yields then the identity,

$$
c_{1}(0)=z_{s}+\left\{a_{1} q \sum_{\ell=2}^{\infty} \gamma_{\ell}+b_{2} \gamma_{2}\right\} / \sum_{\ell=2}^{\infty} a_{\ell} \gamma_{\ell} .
$$

Observe from (2.16) that $c_{1}$ satisfies the inequality,

$$
z_{s} \leq c_{1}(t) \leq 2\left(z_{s}+q / 2^{1 / 3}\right), \quad t>0 .
$$

Hence for any $\delta>0$ we define a space $X_{\delta}$ by

$$
X_{\delta}=\left\{c_{1}:[0, \delta] \rightarrow \mathbf{R}: c_{1}(0) \text { is given by (2.17), } \quad c_{1}\right. \text { is continuous and }
$$




$$
\left.z_{s} \leq c_{1}(t) \leq 2\left(z_{s}+q / 2^{1 / 3}\right), 0 \leq t \leq \delta\right\} .
$$

As previously we equip $X_{\delta}$ with the uniform norm $\|\cdot\|_{\delta}$ and define a mapping $T$ on $X_{\delta}$ by

$$
T c_{1}(t)=z_{s}+\left\{a_{1} q \sum_{\ell=2}^{\infty} c(\ell, t)+b_{2} c(2, t)\right\} / \sum_{\ell=2}^{\infty} a_{\ell} c(\ell, t), 0 \leq t \leq \delta,
$$

where $c(\ell, t), t>0$, is the solution to (2.3) subject to the initial and boundary conditions (2.14).

Lemma 2.2. There exists $\delta_{0}>0$ such that for $0<\delta<\delta_{0}$ the mapping $T$ on $X_{\delta}$ is a contraction.

Proof. We proceed as in Lemma 2.1. We consider $c_{1}, c_{1}^{\prime} \in X_{\delta}$ and for $0 \leq \lambda \leq 1$ we put $c_{\lambda, 1}=\lambda c_{1}+(1-\lambda) c_{1}^{\prime}$. Define $c_{\lambda}(\ell, t), \ell \geq 2,0 \leq t \leq \delta$, to be the solution of (2.3), (2.14) with $c_{\lambda, 1}$ substituted for $c_{1}$. Putting $u_{\lambda}(\ell, t)=\partial c_{\lambda}(\ell, t) / \partial \lambda$ we see from (2.18) that

$$
\begin{aligned}
& T c_{1}(t)-T c_{1}^{\prime}(t)=\int_{0}^{1} d \lambda\left[\left\{\sum_{\ell=2}^{\infty} a_{\ell} c_{\lambda}(\ell, t)\right\}\left\{a_{1} q \sum_{\ell=2}^{\infty} u_{\lambda}(\ell, t)+b_{2} u_{\lambda}(2, t)\right\}\right. \\
& \left.-\left\{a_{1} q \sum_{\ell=2}^{\infty} c_{\lambda}(\ell, t)+b_{2} c_{\lambda}(2, t)\right\}\left\{\sum_{\ell=2}^{\infty} a_{\ell} u_{\lambda}(\ell, t)\right\}\right] /\left[\sum_{\ell=2}^{\infty} a_{\ell} c_{\lambda}(\ell, t)\right]^{2} .
\end{aligned}
$$

Evidently $u_{\lambda}(\ell, t), \ell \geq 2$, satisfies (2.8) with initial and boundary conditions given by

$$
u_{\lambda}(1, t)=0, t \geq 0, u_{\lambda}(\ell, 0)=0, \ell \geq 2 .
$$

Letting $v_{\lambda}(\ell, t)$ be the solution to (2.10) with zero initial and boundary conditions corresponding to (2.20) it follows that (2.12) holds.

We wish to bound the RHS of (2.19) in terms of $\left\|c_{1}-c_{1}^{\prime}\right\|_{\delta}$. To do this we first observe that from (2.10) we have the identity,

$$
\sum_{\ell=2}^{\infty} v_{\lambda}(\ell, t)=-b_{2} \int_{0}^{t} d s v_{\lambda}(2, s)+2\left\|c_{1}-c_{1}^{\prime}\right\|_{\delta} \int_{0}^{t} \sum_{\ell=2}^{\infty} a_{\ell} c_{\lambda}(\ell, s),
$$

whence we conclude that

$$
\left\|\sum_{\ell=2}^{\infty} v_{\lambda}(\ell, \cdot)\right\|_{\delta} \leq 2 \delta\left\|c_{1}-c_{1}^{\prime}\right\|_{\delta}\left\|\sum_{\ell=2}^{\infty} a_{\ell} c_{\lambda}(\ell, \cdot)\right\|_{\delta} .
$$

Now from (2.3) we have that

$$
\sum_{\ell=2}^{\infty} a_{\ell} c_{\lambda}(\ell, t)=\sum_{\ell=2}^{\infty} a_{\ell} \gamma_{\ell}+\int_{0}^{t} d s\left\{a_{2} J(1, c, s)+\sum_{\ell=2}^{\infty}\left[a_{\ell+1}-a_{\ell}\right] J(\ell, c, s)\right\} .
$$

Defining now $g(t), t \geq 0$, by

$$
g(t)=\sum_{\ell=2}^{\infty} a_{\ell} c_{\lambda}(\ell, t),
$$

it follows that there is a constant $K>0$ such that

$$
|g(t)-g(0)| \leq K \int_{0}^{t} g(s) d s, \quad t \geq 0 .
$$


Hence $h(t)=|g(t)-g(0)|$ satisfies the inequality,

$$
h(t) \leq K t g(0)+K \int_{0}^{t} h(s) d s .
$$

We conclude that $h$ satisfies the inequality,

$$
h(t) \leq\left[e^{K t}-1\right] g(0), \quad t \geq 0 .
$$

We may therefore choose $\delta_{0}$ small enough so that $g(0) / 2 \leq g(t) \leq 3 g(0) / 2,0 \leq t \leq$ $\delta_{0}$. Now from (2.12), (2.21) we see that

$$
\left|\left\{a_{1} q \sum_{\ell=2}^{\infty} u_{\lambda}(\ell, t)+b_{2} u_{\lambda}(2, t)\right\} / \sum_{\ell=2}^{\infty} a_{\ell} c_{\lambda}(\ell, t)\right| \leq K_{1} \delta\left\|c_{1}-c_{1}^{\prime}\right\|_{\delta}, 0 \leq t \leq \delta,
$$

for some constant $K_{1}$ independent of $\delta_{0}$, provided $0<\delta<\delta_{0}$.

Next we observe from (2.8) that

$$
\begin{aligned}
\sum_{\ell=2}^{\infty} a_{\ell} u_{\lambda}(\ell, t) & =\int_{0}^{t} d s\left\{a_{2} J\left(1, u_{\lambda}, s\right)+\sum_{\ell=2}^{\infty}\left[a_{\ell+1}-a_{\ell}\right] J\left(\ell, u_{\lambda}, s\right)\right\} \\
& +\int_{0}^{t} d s\left[c_{1}(s)-c_{1}^{\prime}(s)\right] \sum_{\ell=2}^{\infty}\left(a_{\ell+1}-a_{\ell}\right) a_{\ell} c_{\lambda}(\ell, s) .
\end{aligned}
$$

It follows then from (2.12) that there are constants $K_{2}, K_{3}>0$ independent of $\delta_{0}$ such that

$$
\left|\sum_{\ell=2}^{\infty} a_{\ell} u_{\lambda}(\ell, t)\right| \leq K_{1} \delta\left\|\sum_{\ell=2}^{\infty} v_{\lambda}(\ell, \cdot)\right\|_{\delta}+K_{2} \delta\left\|c_{1}-c_{1}^{\prime}\right\|_{\delta}\left\|\sum_{\ell=2}^{\infty} a_{\ell} c_{\lambda}(\ell, \cdot)\right\|_{\delta}, 0 \leq t \leq \delta .
$$

Hence, on using (2.21), we see that there is a constant $K_{4}$ such that

$$
\left|\sum_{\ell=2}^{\infty} a_{\ell} u_{\lambda}(\ell, t)\right| / \sum_{\ell=2}^{\infty} a_{\ell} c_{\lambda}(\ell, t) \leq K_{4} \delta\left\|c_{1}-c_{1}^{\prime}\right\|_{\delta}, 0 \leq t \leq \delta .
$$

It is clear now from (2.19), (2.22), (2.23) that on taking $\delta_{0}$ sufficiently small the mapping $T$ is a contraction for $0<\delta \leq \delta_{0}$.

Theorem 2.2. The system (2.3), 2.14, 2.15) has a unique solution global in time.

Proof. From Lemma 2.2 the solution exists for time $0 \leq t<\delta$. Since the only constraint on the initial condition is (2.15) at $t=0$ and on $c_{1}(t)$ equation (2.17) at $t=0$ we may propagate the solution beyond time $\delta$ since these conditions also hold at $t=\delta$.

It is evident from (2.16) that for a solution to (2.3), (2.14), (2.15) the corresponding function $c_{1}(t)$ satisfies $c_{1}(t)>z_{s}, 0 \leq t<\infty$. We shall show that the $\liminf$ of $c_{1}(t)$ as $t \rightarrow \infty$ is $z_{s}$.

Lemma 2.3. Let $c(\ell, t), \ell \geq 2$, be a solution to (2.3), 2.14), 2.15), and define $g(t)$ by

$$
g(t)=\sum_{\ell=2}^{\infty} c(\ell, t)
$$

Then $g$ is monotonic decreasing and $\lim _{t \rightarrow \infty} g(t)=0$. 
Proof. From (2.3) we see that $g^{\prime}(t)=-b_{2} c(2, t)<0$ whence $g$ is strictly monotone decreasing. From (2.16) there is the inequality.

$$
c_{1}(t) \geq z_{s}+q /\left\langle X^{1 / 3}\right\rangle_{t},
$$

where $\langle\cdot\rangle_{t}$ is the probability measure on the integers $\ell \geq 2$ with weights $c(\ell, t)$. Using the Jensen inequality we have that

$$
\left\langle X^{1 / 3}\right\rangle_{t} \leq\langle X\rangle_{t}^{1 / 3}=1 / g(t)^{1 / 3},
$$

whence we conclude that

$$
c_{1}(t) \geq z_{s}+q g(t)^{1 / 3} .
$$

Suppose now that $g(t)$ does not converge to 0 as $t \rightarrow \infty$. Then there exists $\gamma, T>0$ such that $g(t) \geq \gamma>0$ for $t \geq T$. We shall show that this implies

$$
\lim _{t \rightarrow \infty} \sum_{\ell=2}^{\infty} c(\ell, t)=\infty
$$

contradicting (2.15).

To prove (2.24) we first observe that for any $\ell \geq 2$ one has that

$$
\int_{0}^{\infty} c(\ell, s) d s<\infty, \quad \ell \geq 2 .
$$

For $\ell=2$ the inequality (2.25) evidently follows from the relation $g^{\prime}(t)=-b_{2} c(2, t)$ and the fact that $g$ is always positive. For $\ell=3$ it follows from (2.25) for $\ell=2$ and the fact that $g(t)-c(2, t)$ is bounded for all $t$, and we can proceed by induction to establish it for all $\ell \geq 2$. Now using (2.6) we have that for $t>T$,

$$
\begin{aligned}
\sum_{\ell=2}^{\infty} \ell c(\ell, t) & =1+\int_{T}^{t} d s\left\{-b_{2} c(2, s)+\sum_{\ell=2}^{\infty}\left[a_{\ell} c_{1}(s)-b_{\ell}\right] c(\ell, s)\right\} \\
& \geq K(t)+\frac{1}{2} \int_{T}^{t} d s q \gamma^{1 / 3} g(s),
\end{aligned}
$$

where by (2.25) $K(t)$ is bounded below uniformly in $t$ as $t \rightarrow \infty$. Hence (2.24) holds.

Lemma 2.4. There is the limit, $\liminf _{t \rightarrow \infty} c_{1}(t)=z_{s}$.

Proof. We assume that $\liminf _{t \rightarrow \infty} c_{1}(t)>z_{s}$ and obtain a contradiction. Thus we are assuming that there exists $T, \gamma>0$ such that $c_{1}(t)>z_{s}+\gamma$ for $t \geq T$. Let $f$ be a positive function on the set $\{\ell \in \mathbf{Z}: \ell \geq 2\}$. Then from (2.3) we have that

$$
\begin{aligned}
\frac{d}{d t} \sum_{\ell=2}^{\infty} f(\ell) c(\ell, t) & =f(2) J(1, c, t)+\sum_{\ell=2}^{\infty}[f(\ell+1)-f(\ell)] J(\ell, c, t) \\
& =\left\{\sum_{\ell=2}^{\infty}[f(\ell+1)-f(\ell)] a_{\ell} c_{1}(t)-[f(\ell)-f(\ell-1)] b_{\ell}\right\} c(\ell, t) .
\end{aligned}
$$

where we put $f(1)=0$. We define now $f(\ell)$ by the recurrence,

$$
[f(\ell+1)-f(\ell)] a_{\ell}\left(z_{s}+\gamma\right)-[f(\ell)-f(\ell-1)] b_{\ell}=0, f(1)=0,
$$


for $\ell=2,3, \ldots, L(\gamma)$, where $L(\gamma)$ is an integer depending only on $\gamma$. For $\ell>L(\gamma)$ we define $f(\ell)$ by the formula,

$$
f(\ell)=f(L(\gamma))[\ell / L(\gamma)]^{1 / 3}
$$

It is evident that $L(\gamma)$ can be chosen in such a way that there is the inequality,

$$
[f(\ell+1)-f(\ell)] a_{\ell}\left(z_{s}+\gamma\right)-[f(\ell)-f(\ell-1)] b_{\ell} \geq 0, \ell \geq 2 .
$$

We conclude therefore that

$$
\sum_{\ell=2}^{\infty} f(\ell) c(\ell, t) \geq \sum_{\ell=2}^{\infty} f(\ell) c(\ell, T), \quad t \geq T,
$$

whence it follows that

$$
\liminf _{t \rightarrow \infty} \sum_{\ell=2}^{\infty} \ell^{1 / 3} c(\ell, t)>0 .
$$

Now from (2.16) and Lemma 2.3 we have that $\lim _{t \rightarrow \infty} c_{1}(t)=z_{s}$, a contradiction.

Remark 1. The analogue of Lemma 2.4 for the $L S W$ equation is proved in 14, Proposition 3.2.

\section{Existence and Uniqueness for the Continuous Case}

In this section we prove a global existence theorem for solutions to (1.11) subject to the constraint (1.7), with non-negative measurable initial data satisfying (1.12) and Dirichlet boundary condition $c(0, t)=0, t>0$. We shall also assume without loss of generality that in (1.7) one has $\rho-\rho_{\text {crit }}=1$, and in (1.11) that $\varepsilon=1$. Observe that the parameter $L(t)$ in (1.11) ought to be given in terms of the solution $c(x, t)$ by the formula,

$$
L(t)^{1 / 3}=\int_{0}^{\infty} x^{1 / 3} c(x, t) d x / \int_{0}^{\infty} c(x, t) d x
$$

in order that the conservation law (1.7) holds. Thus $L(t)$ is proportional to the average cluster radius at time $t$.

Let $\mathcal{L}_{t}$ denote the differential operator on the RHS of (1.11) and $\mathcal{L}_{t}^{*}$ its formal adjoint. Thus $\mathcal{L}_{t}^{*}$ is given by the formula

$$
\mathcal{L}_{t}^{*}=(1+x)^{1 / 3} \frac{\partial^{2}}{\partial x^{2}}-\left[1-\left\{\frac{x}{L(t)}\right\}^{1 / 3}\right] \frac{\partial}{\partial x} .
$$

One can estimate the solution of (1.11) by solving the equation $\partial w / \partial t=-\mathcal{L}^{*} w$ backwards in time. Hence if for some $T>0, w(x, t), x>0, t<T$, is the solution with Dirichlet boundary condition $w(0, t)=0$, we have that

$$
\int_{0}^{\infty} w(x, T) c(x, T) d x=\int_{0}^{\infty} w(x, 0) c(x, 0) d x .
$$

It is well known [7] that solutions to parabolic equations can be written as expectation values. In particular let $X(s), s \geq t$, be the solution to the stochastic differential equation,

$$
d X(s)=-\left[1-\left\{\frac{X(s)}{L(s)}\right\}^{1 / 3}\right] d s+\sqrt{2}(1+X(s))^{1 / 6} d W(s)
$$


with the initial condition $X(t)=x$. If $\tau_{x, t}$ is the first hitting time for the process at 0 , then

$$
w(x, t)=E\left[w_{0}(X(T)) ; \tau_{x, t}>T\right], \quad x>0, t<T,
$$

is the solution to the equation $\partial w / \partial t=-\mathcal{L}^{*} w, t<T$, with terminal data $w(x, T)=$ $w_{0}(x)$ and Dirichlet boundary condition $w(0, t)=0$. We shall show that we can control the solution to this terminal-boundary value problem by perturbation theory uniformly for $x>0$ if $L(t)$ has a positive lower bound and for $T-t$ sufficiently small.

Lemma 3.1. Suppose that the parameter $L(t), t \geq 0$, is continuous and satisfies $L(t) \geq L_{0}>0, t \geq 0$, where $L_{0}<1$. Let $w(x, t)$ be the function (3.5) with $w_{0} \equiv 1$. Then there are positive universal constants $C_{1}, C_{2}$ such that

$C_{1} x / \sqrt{T-t} \leq w(x, t) \leq C_{2} x / \sqrt{T-t}, 0 \leq x \leq \sqrt{T-t}, C_{1} \leq w(x, t) \leq 1, x \geq \sqrt{T-t}$, provided $t$ lies in the region $0<T-t<\delta L_{0}^{1 / 2}$, where $\delta>0$ is universal.

Proof. We first show the lower bound on $w$. Since the terminal data $w_{0}$ is positive and monotonic increasing it follows that $w(x, t) \geq u(x, t)$ where $u(x, t)$ satisfies the equation,

$$
\frac{\partial u}{\partial t}+(1+x)^{1 / 3} \frac{\partial^{2} u}{\partial x^{2}}-\frac{\partial u}{\partial x}=0, \quad t<T,
$$

with Dirichlet boundary condition $u(0, t)=0$ and terminal data $u(x, T)=w_{0}(x), x \geq$ 0 . We can estimate the solution of (3.6) using perturbation theory provided $T-t<<$ 1. Let $G(x, t)$ be the fundamental solution of the heat equation,

$$
G(x, t)=\frac{1}{\sqrt{4 \pi t}} \exp \left[\frac{-x^{2}}{4 t}\right], t>0,-\infty<x<\infty .
$$

For $x, y \geq 0, t<T$, we define the kernel $K_{T}(x, y, t)$ by

$$
K_{T}(x, y, t)=G\left(x-y,(1+y)^{1 / 3}(T-t)\right)-G\left(x+y,(1+y)^{1 / 3}(T-t)\right) .
$$

We have that

$$
\begin{gathered}
\frac{\partial K_{T}}{\partial t}+(1+x)^{1 / 3} \frac{\partial^{2} K_{T}}{\partial x^{2}}-\frac{\partial K_{T}}{\partial x}= \\
\left\{a\left(x-y,(1+y)^{1 / 3}(T-t)\right)+\left[(1+x)^{1 / 3}-(1+y)^{1 / 3}\right] b\left(x-y,(1+y)^{1 / 3}(T-t)\right)\right\} \\
G\left(x-y,(1+y)^{1 / 3}(T-t)\right)-\left\{a\left(x+y,(1+y)^{1 / 3}(T-t)\right)+\left[(1+x)^{1 / 3}-(1+y)^{1 / 3}\right]\right. \\
\left.b\left(x+y,(1+y)^{1 / 3}(T-t)\right)\right\} G\left(x+y,(1+y)^{1 / 3}(T-t)\right),
\end{gathered}
$$

where the functions $a, b$ are given by

$$
a(x, t)=x / 2 t, b(x, t)=x^{2} / 4 t^{2}-1 / 2 t .
$$

Consider now the function $v(x, t), x \geq 0, t<T$, defined by

$$
v(x, t)=\int_{0}^{\infty} K_{T}(x, y, t) w_{0}(y) d y .
$$

Then one sees from the previous equation that

$$
\frac{\partial v}{\partial t}+(1+x)^{1 / 3} \frac{\partial^{2} v}{\partial x^{2}}-\frac{\partial v}{\partial x}=g(x, t), x \geq 0, t<T,
$$


where the function $g$ satisfies the inequality,

$$
|g(x, t)| \leq C / \sqrt{T-t}, \quad 0<T-t<1,
$$

for some universal constant $C>0$. We also have that $v(0, t)=0$ and $v(x, T)=$ $w_{0}(x)$. Hence $v(x, t)$ is given by the formula,

$$
v(x, t)=u(x, t)-E\left[\int_{t}^{T} g(X(s), s) d s ; \tau_{x, t}>T\right] .
$$

We conclude therefore that $w(x, t) \geq v(x, t)-2 C \sqrt{T-t}$. From (3.9) we can obtain a lower bound on $v(x, t)$. To see this first observe from (3.8) that

$K_{T}(x, y, t) \geq \eta_{1} G\left(x-y,(1+y)^{1 / 3}(T-t)\right), \quad|x-y| \leq x / 2, x \geq \sqrt{T-t}, T-t \leq 1$,

for some universal $\eta_{1}>0$. Since $(1+x)^{1 / 3}(T-t) \leq 2 x^{2}$ for $x \geq \sqrt{T-t}, T-t \leq 1$, we also have that

$$
\int_{|x-y|<x / 2} G\left(x-y,(1+y)^{1 / 3}(T-t)\right) d y \geq \eta_{2}, \quad x \geq \sqrt{T-t}, T-t \leq 1,
$$

for some universal $\eta_{2}>0$. Hence from (3.12), (3.13) there exists a universal $\eta_{3}>0$ such that $v(x, t) \geq \eta_{3}, x \geq \sqrt{T-t}, T-t \leq 1$. We conclude that $w(x, t) \geq C_{1}>0$ provided $T-t<\delta<1$ for some suitable universal constant $\delta>0$ if $x$ satisfies $x \geq \sqrt{T-t}$.

To conclude the proof of the lower bound we are left to consider the region $0 \leq x \leq \sqrt{T-t}$. We write the solution of (3.10) with zero Dirichlet and terminal conditions $v(0, t)=0, v(x, T)=0$, as a series

$$
\begin{aligned}
v(x, t) & =\sum_{n=0}^{\infty} v_{n}(x, t), \\
v_{n}(x, t) & =-\int_{t}^{T} d s \int_{0}^{\infty} d y K_{s}(x, y, t) g_{n}(y, s), \\
g_{0}=g, g_{n+1} & =g_{n}-\left\{\frac{\partial}{\partial t}+(1+x)^{1 / 3} \frac{\partial^{2}}{\partial x^{2}}-\frac{\partial}{\partial x}\right\} v_{n}, n \geq 0 .
\end{aligned}
$$

The recurrence relation for $g_{n}$ can more simply be written as

$$
g_{n+1}(x, t)=\int_{t}^{T} d s \int_{0}^{\infty} d y\left\{\frac{\partial}{\partial t}+(1+x)^{1 / 3} \frac{\partial^{2}}{\partial x^{2}}-\frac{\partial}{\partial x}\right\} K_{s}(x, y, t) g_{n}(y, s) .
$$

It follows now in exactly the same way that we derived (3.11) that there is a universal constant $C>0$ such that

$$
g_{n}(x, t) \leq C^{n}(T-t)^{n / 2-1 / 2}, \quad n \geq 0 .
$$

Hence the series (3.14) converges for $T-t$ sufficiently small. Observe also that the sum of the $g_{n}, n=0, \ldots, \infty$, is bounded by $C / \sqrt{T-t}$ for some universal constant $C$. Using the fact that

$$
\int_{0}^{\infty} K_{T}(x, y, t) d y \leq C \min [1, x / \sqrt{T-t}],
$$


we can bound the function $v$ in (3.14). Suppose $x=\sqrt{s_{0}-t}$ where $t<s_{0}<$ $t+(T-t) / 2$. Then one has that

$$
\begin{aligned}
& \int_{t}^{T} d s \int_{0}^{\infty} d y K_{s}(x, y, t) / \sqrt{T-s} \leq C \int_{t}^{s_{0}} d s / \sqrt{T-s} \\
&+C x \int_{s_{0}}^{T} d s / \sqrt{(s-t)(T-s)} \leq 2 C\left[\sqrt{T-t}-\sqrt{T-s_{0}}\right] \\
&+\frac{C x \sqrt{2}}{\sqrt{T-t}} \int_{s_{0}}^{(T+t) / 2} \frac{d s}{\sqrt{s-t}}+\frac{C x \sqrt{2}}{\sqrt{T-t}} \int_{(T+t) / 2}^{T} \frac{d s}{\sqrt{T-s}} \leq C^{\prime} x,
\end{aligned}
$$

for some constant $C^{\prime}$. The lower bound follows easily now by obtaining a lower bound on the function (3.9) and using the previous inequality.

We consider next the upper bound. Since it is clear that $w(x, t) \leq 1$ we need only restrict ourselves to $x$ in the region $0 \leq x \leq \sqrt{T-t}$. Let $u(x, t)$ satisfy the equation,

$$
\frac{\partial u}{\partial t}+(1+x)^{1 / 3} \frac{\partial^{2} u}{\partial x^{2}}+\left[\left(\frac{x}{L_{0}}\right)^{1 / 3}-1\right] \frac{\partial u}{\partial x}=0, \quad t<T,
$$

with Dirichlet boundary condition $u(0, t)=0$ and terminal data $u(x, T)=w_{0}(x), x \geq$ 0 . Since the data $w_{0}$ is positive and monotonic increasing we have that $w(x, t) \leq$ $u(x, t)$. To obtain an upper bound on $u(x, t)$ we first note that $u(x, t)=P\left(\tau_{x, t}>T\right)$ where $\tau_{x, t}$ is the first hitting time at 0 for the process (3.4) started at $x$ at time $t$ with $L(s) \equiv L_{0}$. For $0<x<L_{0}^{1 / 4}$ let $\tau_{x, t}^{*}$ be the first exit time from the interval $\left[0, L_{0}^{1 / 4}\right]$. Evidently one has

$$
P\left(\tau_{x, t}>T\right) \leq P\left(\tau_{x, t}^{*}>T\right)+u(x),
$$

where $u(x)$ is the probability that the process started at $x$ exits the interval $\left[0, L_{0}^{1 / 4}\right]$ through the boundary $L_{0}^{1 / 4}$. Now $u(x)$ satisfies the boundary value problem,

$$
(1+x)^{1 / 3} \frac{\partial^{2} u}{\partial x^{2}}+\left[\left(\frac{x}{L_{0}}\right)^{1 / 3}-1\right] \frac{\partial u}{\partial x}=0,0<x<L_{0}^{1 / 4}, u(0)=0, u\left(L_{0}^{1 / 4}\right)=1 .
$$

The problem is explicitly solvable with solution,

$u(x)=\int_{0}^{x} \exp \left[-\int_{0}^{z} h\left(z^{\prime}\right) d z^{\prime}\right] d z / \int_{0}^{L_{0}^{1 / 4}} \exp \left[-\int_{0}^{z} h\left(z^{\prime}\right) d z^{\prime}\right] d z, 0<x<L_{0}^{1 / 4}$,

where $h(z)=\left[\left(z / L_{0}\right)^{1 / 3}-1\right] /[1+z]^{1 / 3}$. Since $L_{0}<1$ we conclude that there is a universal constant $C>0$ such that $u(x) \leq C x / L_{0}^{1 / 4}$. Hence to obtain the upper bound on $w(x, t)$ for $0<x<\sqrt{T-t}$ it is sufficient to obtain an upper bound on $P\left(\tau_{x, t}^{*}>T\right)$.

We can do this by perturbation theory just as we did for the lower bound. Thus setting $u(x, t)=P\left(\tau_{x, t}^{*}>T\right)$ it is clear that $u(x, t)$ satisfies (3.15) for $0<$ $x<L_{0}^{1 / 4}, t<T$, with terminal condition $u(x, T)=1$ and boundary conditions $u(0, t)=u\left(L_{0}^{1 / 4}, t\right)=0$. Let $G_{D}(x, y, t)$ be the Green's function for the heat equation on the interval $\left[0, L_{0}^{1 / 4}\right]$ with Dirichlet boundary conditions. Thus $G_{D}$ is 
given by the method of images as an infinite series,

$$
G_{D}(x, y, t)=\sum_{m=0}^{\infty}(-1)^{p(m)} G\left(x-y_{m}, t\right),
$$

where $y_{0}=y$ and $y_{m}, m \geq 1$, are the multiple reflections of $y$ in the boundaries $0, L_{0}^{1 / 4}$, with $p(m)$ being the parity of the reflection, $p(0)=0$. For $0 \leq x, y \leq L_{0}^{1 / 4}$ and $t<T$ we define the kernel $K_{T}(x, y, t)$ by

$$
K_{T}(x, y, t)=G_{D}\left(x, y,(1+y)^{1 / 3}(T-t)\right) .
$$

We have then that

$$
\begin{gathered}
\text { (3.18) } \frac{\partial K_{T}}{\partial t}+(1+x)^{1 / 3} \frac{\partial^{2} K_{T}}{\partial x^{2}}+\left[\left(\frac{x}{L_{0}}\right)^{1 / 3}-1\right] \frac{\partial K_{T}}{\partial x} \\
=\sum_{m=0}^{\infty}(-1)^{p(m)} G\left(\left(x-y_{m}\right),(1+y)^{1 / 3}(T-t)\right)\left\{a\left(x-y_{m},(1+y)^{1 / 3}(T-t)\right)\left[1-\left(\frac{x}{L_{0}}\right)^{1 / 3}\right]\right. \\
\left.+\left[(1+x)^{1 / 3}-(1+y)^{1 / 3}\right] b\left(x-y_{m},(1+y)^{1 / 3}(T-t)\right)\right\}
\end{gathered}
$$

just as in (3.8). Consider now the function $v(x, t), 0 \leq x \leq L_{0}^{1 / 4}$ defined by

$$
v(x, t)=\int_{0}^{L_{0}^{1 / 4}} K_{T}(x, y, t) d y, \quad t<T .
$$

Then one has that

$$
\frac{\partial v}{\partial t}+(1+x)^{1 / 3} \frac{\partial^{2} v}{\partial x^{2}}+\left[\left(\frac{x}{L_{0}}\right)^{1 / 3}-1\right] \frac{\partial v}{\partial x}=g(x, t),
$$

where the function $g$ satisfies the inequality,

$$
|g(x, t)| \leq C / L_{0}^{1 / 4} \sqrt{T-t} .
$$

Arguing as previously we thus obtain the upper bound on $w(x, t)$ for $0 \leq x \leq$ $\sqrt{T-t}$ provided $T-t<\delta L_{0}^{1 / 2}$ for suitably small $\delta$ independent of $L_{0}$.

Lemma 3.2. Suppose that the parameter $L(t), t \geq 0$, is continuous and satisfies $L(t) \geq L_{0}>0, t \geq 0$, where $L_{0}<1$. Let $w(x, t)$ be the function (3.5) with $w_{0}(x)=x^{1 / 3}, x>0$. Then there are positive universal constants $C_{1}, C_{2}$ such that

$$
\begin{aligned}
C_{1} x /(T-t)^{1 / 3} & \leq w(x, t) \leq C_{2} x /(T-t)^{1 / 3}, 0 \leq x \leq \sqrt{T-t}, \\
C_{1} x^{1 / 3} & \leq w(x, t) \leq C_{2} x^{1 / 3}, x \geq \sqrt{T-t},
\end{aligned}
$$

provided $t$ lies in the region $0<T-t<\delta L_{0}^{1 / 2}$, where $\delta>0$ is universal.

Proof. The proof of the lower bound is similar to the proof of the lower bound in Lemma 3.1. For the proof of the upper bound however we need to make a modification since $w_{0}(x)=x^{1 / 3}$ is an unbounded function. Our starting point is as before that we wish to find an upper bound on the solution to (3.15). Then if $X(s)$ is the stochastic process started at $x$ at time $t$ which is associated with the PDE (3.15), we have that

$$
u(x, t)=E\left[w_{0}(X(T)) ; \tau_{x, t}>T\right]
$$




$$
\leq E\left[w_{0}(X(T)) ; \tau_{x, t}^{*}>T\right]+u(x) \sup _{t<t^{\prime}<T} u\left(L_{0}^{1 / 4}, t^{\prime}\right),
$$

using the notation of Lemma 3.1. The first term on the right can be bounded above using perturbation theory as before. We shall therefore be finished if we can show that $u\left(L_{0}^{1 / 4}, t^{\prime}\right) \leq C L_{0}^{1 / 12}$ for $t<t^{\prime}<T$, provided $T-t<L_{0}^{1 / 2}$. We can use Ito calculus to prove this. Thus we have that

$$
d X(s)=\left[\left\{\frac{X(s)}{L_{0}}\right\}^{1 / 3}-1\right] d s+\sqrt{2}(1+X(s))^{1 / 6} d W(s) .
$$

It follows that if $X(t)=x$, then for $t^{\prime}>t$,

$$
\begin{aligned}
X\left(t^{\prime}\right)^{2} & =x^{2}+2 \int_{t}^{t^{\prime}} X(s)\left[\left\{\frac{X(s)}{L_{0}}\right\}^{1 / 3}-1\right] d s \\
& +2 \int_{t}^{t^{\prime}}(1+X(s))^{1 / 3} d s+2 \sqrt{2} \int_{t}^{t^{\prime}} X(s)(1+X(s))^{1 / 6} d W(s) .
\end{aligned}
$$

Setting $\tau=\tau_{x, t}$ we conclude that

$E\left[X\left(t^{\prime} \wedge \tau\right)^{2}\right]=x^{2}+E\left[\int_{t}^{t^{\prime} \wedge \tau} X(s)\left[\left\{\frac{X(s)}{L_{0}}\right\}^{1 / 3}-1\right] d s\right]+2\left[\int_{t}^{t^{\prime} \wedge \tau}(1+X(s))^{1 / 3} d s\right]$.

We take now $x=L_{0}^{1 / 4}$ and restrict $t^{\prime}$ by $t^{\prime}-t<L_{0}^{1 / 2}$. It follows that there is the inequality,

$$
E\left[X\left(t^{\prime} \wedge \tau\right)^{2}\right] \leq C_{1} L_{0}^{1 / 2}+C_{2} L_{0}^{-1 / 2} E\left[\int_{t}^{t^{\prime} \wedge \tau} X(s)^{2} d s\right]
$$

for some universal constants $C_{1}, C_{2}$. Gronwall's inequality therefore yields,

$$
E\left[X\left(t^{\prime} \wedge \tau\right)^{2}\right] \leq C_{1} L_{0}^{1 / 2} \exp \left[C_{2}\left(t^{\prime}-t\right) / L_{0}^{1 / 2}\right] .
$$

We can now apply the Chebyshev inequality to conclude that $u\left(L_{0}^{1 / 4}, t^{\prime}\right) \leq C L_{0}^{1 / 12}$.

Lemma 3.3. Let $w(x, t)$ be the function (3.5) with $w_{0} \equiv 1$. Then $0 \leq \partial w(x, t) / \partial x \leq$ $C / \sqrt{T-t}$, provided $0<(T-t)<\delta L_{0}^{1 / 2}$.

Proof. We have already observed that $0 \leq \partial w(x, t) / \partial x$ so we need an upper bound. We first prove the upper bound for $0 \leq x \leq L_{0}^{1 / 4} / 2$. To do this we observe that $w(x, t), 0 \leq x \leq L_{0}^{1 / 4}, t<T$, satisfies the diffusion equation with terminal conditions $w_{0}(x)=1,0 \leq x \leq L_{0}^{1 / 4}$, and boundary conditions $w(0, t)=$ $0, w\left(L_{0}^{1 / 4}, t\right) \leq 1, t<T$. We write $w(x, t)=w_{1}(x, t)+w_{2}(x, t)$ where both $w_{1}$ and $w_{2}$ satisfy the diffusion equation. The function $w_{1}(x, t)$ has terminal data $w_{1}(x, T)=w_{0}(x), 0 \leq x \leq L_{0}^{1 / 4}$, and boundary data $w_{1}(0, t)=w_{1}\left(L_{0}^{1 / 4}, t\right)=0$. The function $w_{2}(x, t)$ has terminal data $w_{2}(x, T)=0,0 \leq x \leq L_{0}^{1 / 4}$, and boundary data $w_{2}(0, t)=0, w_{2}\left(L_{0}^{1 / 4}, t\right)=w\left(L_{0}^{1 / 4}, t\right)$.

We first consider the function $w_{1}(x, t)$ which we construct by perturbation expansion. The first term in the series is the function $v(x, t)$ of (3.19). Setting 


$$
\begin{aligned}
& g(x, t)=\partial v / \partial t+\mathcal{L}_{t}^{*} v, \text { then } \\
& w_{1}(x, t)= v(x, t)-\sum_{n=0}^{\infty} v_{n}(x, t), \\
& v_{n}(x, t)=-\int_{t}^{T} d s \int_{0}^{L_{0}^{1 / 4}} d y K_{s}(x, y, t) g_{n}(y, s), \\
& g_{0}=g, \quad g_{n+1}=g_{n}-\left\{\frac{\partial}{\partial t}+\mathcal{L}_{t}^{*}\right\} v_{n}, n \geq 0 .
\end{aligned}
$$

Just as before we see that the functions $g_{n}$ satisfy the inequality,

$$
\left|g_{n}(x, t)\right| \leq C^{n}(T-t)^{n / 2-1 / 2} / L_{0}^{(n+1) / 4}, \quad n \geq 0 .
$$

It easily follows that $\left|\partial w_{1} / \partial x\right| \leq C_{1} / \sqrt{T-t}$ for some constant $C_{1}$ provided $0<$ $T-t<\delta L_{0}^{1 / 2}$.

Next we consider the function $w_{2}(x, t)$. This can be represented in terms of a Green's function $G(x, y, t, T), 0 \leq x, y \leq L_{0}^{1 / 4}, t<T$, which is the Dirichlet Green's function for the operator $\mathcal{L}_{t}^{*}$ on the interval $\left[0, L_{0}^{1 / 4}\right]$. Thus if

$$
u(x, t)=\int_{0}^{L_{0}^{1 / 4}} G(x, y, t, T) w_{0}(y) d y, \quad t<T,
$$

then

$$
\begin{aligned}
\frac{\partial u}{\partial t}+\mathcal{L}_{t}^{*} u=0,0<x<L_{0}^{1 / 4}, \quad t<T, & \\
& u(0, t)=u\left(L_{0}^{1 / 4}, t\right)=0, \quad u(x, T)=w_{0}(x), 0<x<L_{0}^{1 / 4} .
\end{aligned}
$$

The function $w_{2}(x, t), t<T$, has the representation,

$$
w_{2}(x, t)=-\int_{t}^{T} d s w\left(L_{0}^{1 / 4}, s\right) \frac{\partial G}{\partial y}\left(x, L_{0}^{1 / 4}, t, s\right)\left[1+L_{0}^{1 / 4}\right]^{1 / 3} .
$$

To estimate $w_{2}(x, t)$ we compute the Green's function by perturbation series expansion as we did before. The first term in the expansion for $G(x, y, t, T)$ is the function $K_{T}(x, y, t)$ of (3.17). If we replace $G$ by this in (3.22) it is easy to see that $\left|\partial w_{2} / \partial x\right| \leq C(T-t) / L_{0}^{3 / 4}, 0<x<L_{0}^{1 / 4} / 2$. The complete expansion for $G(x, y, t, T)$ is given by

$$
\begin{aligned}
G(x, y, t, T) & =K_{T}(x, y, t)-\sum_{n=0}^{\infty} v_{n, T}(x, y, t), \\
v_{n, T}(x, y, t) & =-\int_{t}^{T} d s \int_{0}^{L_{0}^{1 / 4}} d y^{\prime} K_{s}\left(x, y^{\prime}, t\right) g_{n, T}\left(y^{\prime}, y, s\right), \\
g_{0, T}(x, y, t) & =g_{T}(x, y, t)=\left[\frac{\partial}{\partial t}+\mathcal{L}_{t, x}^{*}\right] K_{T}(x, y, t), \\
g_{n+1, T} & =g_{n, T}-\left\{\frac{\partial}{\partial t}+\mathcal{L}_{t}^{*}\right\} v_{n, T}, n \geq 0, \\
g_{n+1, T}(x, y, t) & =\int_{t}^{T} d s \int_{0}^{L_{0}^{1 / 4}} d y^{\prime}\left\{\frac{\partial}{\partial t}+\mathcal{L}_{t, x}^{*}\right\} K_{s}\left(x, y^{\prime}, t\right) g_{n, T}\left(y^{\prime}, y, s\right) .
\end{aligned}
$$


In (3.23) we have written $\mathcal{L}_{t}^{*}=\mathcal{L}_{t, x}^{*}$ to denote that the operator $\mathcal{L}_{t}^{*}$ acts on the $x$ variable. Analogously to (3.21) we have the estimate

$$
\left|g_{n, T}(x, y, t)\right| \leq \frac{C^{n}(T-t)^{n / 2}-1 / 2}{L_{0}^{(n+1) / 4}} G\left(x-y,\left(1+L_{0}^{1 / 4}\right)^{1 / 3}(T-t)\right),
$$

for some universal constant $C$. Hence the series (3.23) converges for $0<T-t<$ $\delta L_{0}^{1 / 2}$. We need now to differentiate the series term by term with respect to the $y$ variable. Consider first the function $v_{0, T}$ whose derivative is formally given by the expression

$$
\frac{\partial v_{0, T}}{\partial y}(x, y, t)=-\int_{t}^{T} d s \int_{0}^{L_{0}^{1 / 4}} d y^{\prime} K_{s}\left(x, y^{\prime}, t\right) \frac{\partial g_{T}}{\partial y}\left(y^{\prime}, y, s\right) .
$$

Analogously to (3.24) one has the estimate,

$$
\left|\frac{\partial g_{T}(x, y, t)}{\partial y}\right| \leq \frac{C}{(T-t) L_{0}^{1 / 4}} G\left(x-y,\left(1+L_{0}^{1 / 4}\right)^{1 / 3}(T-t)\right) .
$$

This estimate gives a nonintegrable singularity in (3.25), so we need to integrate by parts in (3.25) in the $s, y^{\prime}$ variables. First we write

$$
\begin{aligned}
& \frac{\partial v_{0, T}}{\partial y}(x, y, t)=-\int_{t}^{(T+t) / 2} d s \int_{0}^{L_{0}^{1 / 4}} d y^{\prime} K_{s}\left(x, y^{\prime}, t\right) \frac{\partial g_{T}}{\partial y}\left(y^{\prime}, y, s\right) \\
& -\int_{(T+t) / 2}^{T} d s \int_{0}^{L_{0}^{1 / 4}} K_{s}\left(x, y^{\prime}, t\right)\left\{\frac{\partial}{\partial s}+\mathcal{L}_{s, y^{\prime}}^{*}\right\} \frac{\partial K_{T}}{\partial y}\left(y^{\prime}, y, s\right) .
\end{aligned}
$$

We have now from (3.17), (3.18) that

$$
\begin{gathered}
\left\{\frac{\partial}{\partial s}+\mathcal{L}_{s, y^{\prime}}^{*}\right\} \frac{\partial K_{T}}{\partial y}\left(y^{\prime}, y, s\right)=\left[\left\{\frac{y^{\prime}}{L(s)}\right\}^{1 / 3}-1\right] \frac{\partial^{2} K_{T}}{\partial y^{\prime} \partial y} \\
+\frac{\left(1+y^{\prime}\right)^{1 / 3}}{3(1+y)^{4 / 3}} \frac{\partial K_{T}}{\partial s}+\left[1-\left(\frac{1+y^{\prime}}{1+y}\right)^{1 / 3}\right] \frac{\partial^{2} K_{T}}{\partial s \partial y} .
\end{gathered}
$$

We substitute the RHS of (3.27) into the second integral on the RHS of (3.26). We then integrate by parts w.r. to $y^{\prime}$ for the first term on the RHS of (3.27), and w.r. to $s$ for the second two terms. Note that since $K_{s}(x, 0, t)=K_{s}\left(x, L_{0}^{1 / 4}, t\right)=0$ there are no boundary terms in the integration by parts w.r. to $y^{\prime}$. Once this is accomplished we can estimate $\partial v_{0, T} / \partial y$ since we have removed all non integrable singularities. Thus we obtain the bound,

$$
\left|\frac{\partial v_{0, T}}{\partial y}(x, y, t)\right| \leq \frac{C}{L_{0}^{1 / 4}} G\left(x-y,\left(1+L_{0}^{1 / 4}\right)^{1 / 3}(T-t)\right) .
$$

Similarly we also have that

$$
\left|\frac{\partial g_{1, T}}{\partial y}(x, y, t)\right| \leq \frac{C}{L_{0}^{1 / 2} \sqrt{T-t}} G\left(x-y,\left(1+L_{0}^{1 / 4}\right)^{1 / 3}(T-t)\right) .
$$

Once we have the estimate of (3.29) it follows by the same method as was used to derive (3.24) that

$$
\left|\frac{\partial g_{n, T}}{\partial y}(x, y, t)\right| \leq \frac{C^{n}(T-t)^{n / 2-1}}{L_{0}^{(n+1) / 4}} G\left(x-y,\left(1+L_{0}^{1 / 4}\right)^{1 / 3}(T-t)\right), n \geq 1 .
$$


Hence from (3.23) we also have that

$$
\left|\frac{\partial^{2} v_{n, T}}{\partial x \partial y}(x, y, t)\right| \leq \frac{C^{n}(T-t)^{n / 2-1 / 2}}{L_{0}^{(n+1) / 4}} G\left(x-y,\left(1+L_{0}^{1 / 4}\right)^{1 / 3}(T-t)\right), n \geq 1 .
$$

Now, just as we derived (3.28) we can see that 3.31 also holds for $n=0$. We conclude therefore that there is a universal constant $C$ such that

$$
\left|\frac{\partial^{2} G}{\partial x \partial y}\left(x, L_{0}^{1 / 4}, t, s\right)\right| \leq \frac{C}{L_{0}^{3 / 4}}, \quad 0<x<L_{0}^{1 / 4} / 2,0<s-t<\delta L_{0}^{1 / 4} .
$$

It follows then from (3.22) that one has $\left|\partial w_{2}(x, t) / \partial x\right| \leq C / L_{0}^{1 / 4}, \quad 0<x<$ $L_{0}^{1 / 4} / 2, T-t<\delta L_{0}^{1 / 4}$.

We consider next the case $x \geq L_{0}^{1 / 4} / 2$. First note that we have shown that $\partial w(x, t) / \partial x \leq C / L_{0}^{1 / 4}, x=L_{0}^{1 / 4} / 2, T-t<\delta L_{0}^{1 / 4}$. Thus if $v(x, t)=\partial w(x, t) / \partial x, x>$ $L_{0}^{1 / 4} / 2, t<T$, then it is clear that $v$ is the solution to the terminal- boundary value problem,

$$
\begin{aligned}
& \frac{\partial v}{\partial t}+(1+x)^{1 / 3} \frac{\partial^{2} v}{\partial x^{2}}+\left[\frac{1}{3(1+x)^{2 / 3}}+\left\{\frac{x}{L(t)}\right\}^{1 / 3}-1\right] \frac{\partial v}{\partial x} \\
& \quad+\frac{1}{3\left\{x^{2} L(t)\right\}^{1 / 3}} v=0, \quad t<T, x>L_{0}^{1 / 4} / 2, \\
& v\left(L_{0}^{1 / 4} / 2, t\right)=\partial w / \partial x\left(L_{0}^{1 / 4} / 2, t\right), \quad t<T ; \quad v(x, T)=0, \quad x \geq L_{0}^{1 / 4} / 2 .
\end{aligned}
$$

Let $X(s)$ denote the diffusion corresponding to the equation (3.32) and for $X(t)=x>L_{0}^{1 / 4} / 2$, let $\tau_{x, t}$ be the first hitting time at $L_{0}^{1 / 4} / 2$. Then there is the inequality,

$$
v(x, t) \leq \frac{C}{L_{0}^{1 / 4}} E\left[\exp \left\{\int_{t}^{T \wedge \tau_{x, t}} \frac{d s}{3\left[X(s)^{2} L(s)\right]^{1 / 3}}\right\} \mid X(t)=x\right] .
$$

In view of the fact that $T-t<\delta L_{0}^{1 / 2}$ and $X(s) \geq L_{0}^{1 / 4} / 2, t<s<T \wedge \tau_{x, t}$, we conclude that $v(x, t) \leq C_{1} / L_{0}^{1 / 4}$ for some universal constant $C_{1}$ if $x \geq L_{0}^{1 / 4} / 2$.

Lemma 3.4. Let $w(x, t)$ be the function 3.5 with $w_{0}(x)=x^{1 / 3}, x>0$. Then $0 \leq \partial w(x, t) / \partial x \leq C /(T-t)^{1 / 3}$, provided $0<(T-t)<\delta L_{0}^{1 / 2}$.

Proof. We proceed exactly as in Lemma 3.3 but this time using the results of Lemma 3.2. Thus we first show that $\partial w(x, t) / \partial x \leq C /(T-t)^{1 / 3}$ for $x \leq L_{0}^{1 / 4} / 2$ and $\partial w(x, t) / \partial x \leq C / L_{0}^{1 / 6}$ for $x=L_{0}^{1 / 4} / 2$. For $x>L_{0}^{1 / 4} / 2$ we consider the terminal-boundary value problem (3.32) but now with the terminal data given by $v(x, T)=1 / 3 x^{2 / 3}$. Since $v(x, T) \leq C_{1} / L_{0}^{1 / 6}, x \geq L_{0}^{1 / 4} / 2$, one concludes just as in Lemma 3.3 that $\partial w(x, t) / \partial x \leq C / L_{0}^{1 / 6}, x \geq L_{0}^{1 / 4} / 2$.

Theorem 3.1. Suppose $c(x, 0), x>0$, is a non negative function which satisfies,

$$
0<\int_{0}^{\infty}(1+x) c(x, 0) d x<\infty
$$


Then there is a unique solution to (1.11) subject to the constraint,

$$
\int_{0}^{\infty} x c(x, t) d x=\int_{0}^{\infty} x c(x, 0) d x .
$$

Proof. Just as in $\S 2$ we define a mapping on functions $c_{1}(T)=1 / L(T)^{1 / 3}$ where $L(T)$ is defined by (3.1). In particular, $c_{1}(0)$ is determined by the initial data, where $c_{1}(0)=1 / L_{1}^{1 / 3}$ for some $L_{1}>0$. Suppose now we are given $c_{1}(T), 0 \leq T \leq$ $T_{0}$, with $c_{1}(0)=1 / L_{1}^{1 / 3}$. Then we solve (1.11) with the corresponding function $L(T), 0 \leq T \leq T_{0}$, and define a new function $A c_{1}(T), 0 \leq T \leq T_{0}$, by the RHS of (3.1). We define a space $X$ of functions $c_{1}:\left[0, T_{0}\right] \rightarrow(0, \infty)$ which are continuous and satisfy $\left\|c_{1}\right\|_{\infty} \leq 1 / L_{0}^{1 / 3}$ for some $L_{0}>0$. We shall show that the mapping $A$ leaves $X$ invariant provided $L_{0}<L_{1}$ is sufficiently small and $T_{0}=\delta L_{0}^{1 / 2}$ for some universal $\delta, 0<\delta<1$. To see this we write

$$
\begin{aligned}
A c_{1}(T) & =\int_{0}^{\infty} c(x, T) d x / \int_{0}^{\infty} x^{1 / 3} c(x, T) d x \\
& =\int_{0}^{\infty} w_{1}(x, 0) c(x, 0) d x / \int_{0}^{\infty} w_{2}(x, 0) c(x, 0) d x
\end{aligned}
$$

where $w_{1}(x, t), t<T$, is given by 3.5 with $w_{0} \equiv 1$, and $w_{2}(x, t), t<T$, is given by (3.5) with $w_{0}(x)=x^{1 / 3}, x>0$. In view of Lemma 3.1 and 3.2 we have then that

$$
\begin{gathered}
A c_{1}(T) \leq\left[\frac{C_{2}}{\sqrt{T}} \int_{0}^{\sqrt{T}} x c(x, 0) d x+\int_{\sqrt{T}}^{\infty} c(x, 0) d x\right] / \\
{\left[\frac{C_{1}}{T^{1 / 3}} \int_{0}^{\sqrt{T}} x c(x, 0) d x+C_{1} \int_{\sqrt{T}}^{\infty} x^{1 / 3} c(x, 0) d x\right],}
\end{gathered}
$$

provided $T<\delta L_{0}^{1 / 2}$, for some universal $\delta>0$. Observe now that

$$
\begin{aligned}
\int_{0}^{\infty} x^{1 / 3} c(x, 0) d x & \leq \frac{L_{1}^{1 / 3}}{2} \int_{0}^{L_{1} / 8} c(x, 0) d x+\int_{L_{1} / 8}^{\infty} x^{1 / 3} c(x, 0) d x \\
& \leq \frac{1}{2} \int_{0}^{\infty} x^{1 / 3} c(x, 0) d x+\int_{L_{1} / 8}^{\infty} x^{1 / 3} c(x, 0) d x
\end{aligned}
$$

whence we conclude that

$$
\int_{L_{1} / 8}^{\infty} x^{1 / 3} c(x, 0) d x \geq \frac{1}{2} \int_{0}^{\infty} x^{1 / 3} c(x, 0) d x .
$$

It follows then from (3.34) that

$$
A c_{1}(T) \leq 2 \max \left[C_{2}, 1\right] / C_{1} L_{1}^{1 / 3}, T<L_{1}^{2} / 64 .
$$

One also has from (3.34) that

$$
A c_{1}(T) \leq\left[C_{2} / C_{1}+1 / C_{1}\right] / T^{1 / 6}, 0<T<\delta L_{0}^{1 / 2} .
$$

It is clear now that there is a universal constant $\eta>0$ such that if we choose $L_{0}=\eta \min \left[L_{1}, 1\right]$ then $A$ leaves $X$ invariant. Next we show that for $T_{0}$ sufficiently 
small the mapping $A$ is a contraction. To see this we proceed as in $\S 2$. Thus if $c_{1}, c_{1}^{\prime} \in X$ then

$$
\begin{aligned}
A c_{1}(T) & -A c_{1}^{\prime}(T)=\int_{0}^{1} d \lambda \frac{\partial}{\partial \lambda} A\left[\lambda c_{1}+(1-\lambda) c_{1}^{\prime}\right](T) \\
& =\int_{0}^{1} d \lambda\left\{\int_{0}^{\infty} x^{1 / 3} c_{\lambda}(x, T) d x \int_{0}^{\infty} \frac{\partial c_{\lambda}}{\partial \lambda}(x, T) d x\right. \\
& \left.-\int_{0}^{\infty} c_{\lambda}(x, T) d x \int_{0}^{\infty} x^{1 / 3} \frac{\partial c_{\lambda}}{\partial \lambda}(x, T) d x\right\} /\left[\int_{0}^{\infty} x^{1 / 3} c_{\lambda}(x, T) d x\right]^{2},
\end{aligned}
$$

where $c_{\lambda}$ is the solution to (1.11) with $1 / L(t)^{1 / 3}=\lambda c_{1}(t)+(1-\lambda) c_{1}^{\prime}(t)$. Hence $u_{\lambda}=\partial c_{\lambda} / \partial \lambda$ is the solution to the initial-boundary value problem,

$$
\begin{gathered}
\frac{\partial u_{\lambda}}{\partial t}=\mathcal{L}_{t} u_{\lambda}-\left[c_{1}(t)-c_{1}^{\prime}(t)\right] \frac{\partial}{\partial x}\left\{x^{1 / 3} c_{\lambda}\right\}, \quad t>0, \\
u_{\lambda}(x, 0)=0, x>0, \quad u_{\lambda}(0, t)=0, \quad t>0 .
\end{gathered}
$$

It follows that

$$
\begin{aligned}
\int_{0}^{\infty} u_{\lambda}(x, T) d x & =\int_{0}^{T}\left[c_{1}(t)-c_{1}^{\prime}(t)\right] d t \int_{0}^{\infty} d x \frac{\partial w_{1}}{\partial x}(x, t) x^{1 / 3} c_{\lambda}(x, t), \\
\int_{0}^{\infty} x^{1 / 3} u_{\lambda}(x, T) d x & =\int_{0}^{T}\left[c_{1}(t)-c_{1}^{\prime}(t)\right] d t \int_{0}^{\infty} d x \frac{\partial w_{2}}{\partial x}(x, t) x^{1 / 3} c_{\lambda}(x, t),
\end{aligned}
$$

where the functions $w_{1}, w_{2}$ are as in (3.33). Hence from Lemma 3.3 there is a universal constant $C$ such that

$$
\left|\int_{0}^{\infty} u_{\lambda}(x, T) d x / \int_{0}^{\infty} x^{1 / 3} c_{\lambda}(x, T) d x\right| \leq C \gamma(T) \sqrt{T}\left\|c_{1}-c_{1}^{\prime}\right\|_{\infty}, 0<T<\delta L_{0}^{1 / 2}
$$

where $\gamma(T)$ is defined by

$$
\gamma(T)=\sup _{0<t<T} \int_{0}^{\infty} x^{1 / 3} c_{\lambda}(x, t) d x / \int_{0}^{\infty} x^{1 / 3} c_{\lambda}(x, T) d x .
$$

If we use the second equation in (3.37) and (3.36) we also conclude there is a universal constant $C$ such that

$$
\begin{aligned}
& \mid \int_{0}^{\infty} c_{\lambda}(x, T) d x \int_{0}^{\infty} x^{1 / 3} u_{\lambda}(x, T) d x /\left[\int_{0}^{\infty} x^{1 / 3} c_{\lambda}(x, T) d x\right]^{2} \mid \\
& \leq C \gamma(T) \sqrt{T}\left\|c_{1}-c_{1}^{\prime}\right\|_{\infty}, \quad 0<T<\delta L_{0}^{1 / 2} .
\end{aligned}
$$

Using (3.35) and Lemma 3.2 we see that there is a universal constant $C$ such that

$$
\gamma(T) \leq C \max \left[\left(T / L_{1}^{2}\right)^{1 / 3}, 1\right], \quad 0<T<\delta L_{0}^{1 / 2} .
$$

It follows now from (3.38), (3.39) that if we take $T_{0}=\nu \min \left[L_{0}^{1 / 2}, L_{1}^{4 / 5}\right]$ for some universal constant $\nu>0$ then the mapping $A$ is a contraction. Hence we obtain a unique solution to the diffusive LSW problem up to time $T_{0}$. Observe also that for sufficiently small $L_{1}$ one has that $A c_{1}\left(T_{0}\right) \sim 1 / L_{1}^{2 / 15}<1 / L_{1}^{1 / 3}$, whence one obtains global existence in time. 
We show here that the Kohn-Otto argument 8 may be applied to prove time averaged coarsening for the diffusive LSW model (1.7), (1.11). Letting $c_{\varepsilon}(x, t)$ be the solution to the diffusive LSW system (1.7), (1.11), we denote by $E_{\varepsilon}(t)$ the energy

$$
E_{\varepsilon}(t)=\int_{0}^{\infty} x^{2 / 3} c_{\varepsilon}(x, t) d x .
$$

From the conservation law (1.7), the quantity $1 / E_{\varepsilon}(T)^{3}$ is a measure of the average cluster volume at time $T$.

Theorem 3.2. Let $\rho-\rho_{\text {crit }}$ in (1.7) be normalized to 1 , and $c_{0}(x), x \geq 0$, be a non-negative function which satisfies

$$
\int_{0}^{\infty}\left(1+x^{4 / 3}\right) c_{0}(x) d x=M_{0}<\infty .
$$

Suppose further that $c_{\varepsilon}(x, t)$ is the solution of the diffusive LSW system (1.7), (1.11) with initial data $c_{\varepsilon}(x, 0)=c_{0}(x), x \geq 0$. Then for $0<\varepsilon \leq 1$, there are universal constants $K_{1}, K_{2}$ such that

$$
\left[\frac{1}{T} \int_{0}^{T} E_{\varepsilon}(t)^{2} d t\right]^{-3 / 2} \leq K_{1} T,
$$

provided $T \geq K_{2} M_{0}^{3}$.

Proof. We first show that $E_{\varepsilon}(t)$ is decreasing. In fact we have from (1.11) and (3.1) that

$$
\begin{aligned}
\frac{3}{2} \frac{d E}{d t}=-\frac{\varepsilon}{3} \int_{0}^{\infty} x^{-4 / 3}(1 & +x / \varepsilon)^{1 / 3} c_{\varepsilon}(x, t) d x-\int_{0}^{\infty} x^{-1 / 3} c_{\varepsilon}(x, t) d x \\
+ & {\left[\int_{0}^{\infty} c_{\varepsilon}(x, t) d x\right]^{2} / \int_{0}^{\infty} x^{1 / 3} c_{\varepsilon}(x, t) d x<0 . }
\end{aligned}
$$

Next define a length scale $M_{\varepsilon}(t)$ by

$$
M_{\varepsilon}(t)=\int_{0}^{\infty} x^{4 / 3} c_{\varepsilon}(x, t) d x
$$

so that $M_{\varepsilon}(0) \leq M_{0}$. It follows again from (1.11) that

$\frac{3}{4} \frac{d M_{\varepsilon}}{d t}=\frac{\varepsilon}{3} \int_{0}^{\infty} x^{-2 / 3}(1+x / \varepsilon)^{1 / 3} c_{\varepsilon}(x, t) d x+\int_{0}^{\infty} x^{1 / 3}\left[\left\{\frac{x}{L_{\varepsilon}(t)}\right\}^{1 / 3}-1\right] c_{\varepsilon}(x, t) d x$,

where $L_{\varepsilon}(t)$ is given by (3.1) with $c_{\varepsilon}(\cdot, t)$ in place of $c(\cdot, t)$. Now just as in [4] we have from the Schwarz inequality,

$$
\begin{gathered}
{\left[\int_{0}^{\infty} x^{1 / 3}\left[\left\{\frac{x}{L_{\varepsilon}(t)}\right\}^{1 / 3}-1\right] c_{\varepsilon}(x, t) d x\right]^{2} \leq \int_{0}^{\infty} x c_{\varepsilon}(x, t) d x \int_{0}^{\infty} x^{-1 / 3}\left[\left\{\frac{x}{L_{\varepsilon}(t)}\right\}^{1 / 3}-1\right]^{2} c_{\varepsilon}(x, t) d x} \\
=\int_{0}^{\infty} x^{-1 / 3} c_{\varepsilon}(x, t) d x-\left[\int_{0}^{\infty} c_{\varepsilon}(x, t) d x\right]^{2} / \int_{0}^{\infty} x^{1 / 3} c_{\varepsilon}(x, t) d x,
\end{gathered}
$$

where we have used (1.7), (3.1). 
We show that for $0<\varepsilon \leq 1$ there is a constant $C>0$ such that (3.47)

$$
\left[\varepsilon \int_{0}^{\infty} x^{-2 / 3}(1+x / \varepsilon)^{1 / 3} c_{\varepsilon}(x, t) d x\right]^{2} \leq C \varepsilon \int_{0}^{\infty} x^{-4 / 3}(1+x / \varepsilon)^{1 / 3} c_{\varepsilon}(x, t) d x .
$$

To see this observe that the LHS is bounded above by

$$
\begin{aligned}
& 2\left[\varepsilon \int_{0}^{1} x^{-2 / 3}(1+x / \varepsilon)^{1 / 3} c_{\varepsilon}(x, t) d x\right]^{2}+2\left[\varepsilon \int_{1}^{\infty} x^{-2 / 3}(1+x / \varepsilon)^{1 / 3} c_{\varepsilon}(x, t) d x\right]^{2} \\
\leq & 2\left[\varepsilon^{2} \int_{0}^{1} x^{-4 / 3}(1+x / \varepsilon)^{2 / 3} c_{\varepsilon}(x, t) d x\right]\left[\int_{0}^{1} c_{\varepsilon}(x, t) d x\right] \\
+ & 2\left[\varepsilon^{2} \int_{1}^{\infty} x^{-7 / 3}(1+x / \varepsilon)^{2 / 3} c_{\varepsilon}(x, t) d x\right]\left[\int_{1}^{\infty} x c_{\varepsilon}(x, t) d x\right] \\
\leq & 2^{4 / 3} \varepsilon^{2 / 3}\left[\varepsilon \int_{0}^{\infty} x^{-4 / 3}(1+x / \varepsilon)^{1 / 3} c_{\varepsilon}(x, t) d x\right]\left[\int_{0}^{\infty}(1+x) c_{\varepsilon}(x, t) d x\right] \\
\leq & 2^{4 / 3}\left[\varepsilon \int_{0}^{\infty} x^{-4 / 3}(1+x / \varepsilon)^{1 / 3} c_{\varepsilon}(x, t) d x\right]\left[\int_{0}^{\infty}(1+x) c_{\varepsilon}(x, 0) d x\right] .
\end{aligned}
$$

We conclude then from (3.43)-(3.47) that

$$
\left|d M_{\varepsilon} / d t\right|^{2} \leq K_{3}\left|d E_{\varepsilon} / d t\right|,
$$

for some universal constant $K_{3}$, provided $0<\varepsilon \leq 1$. It is easy to see from the Schwarz inequality that

$$
E_{\varepsilon}(t) M_{\varepsilon}(t) \geq 1, \quad t>0 .
$$

The result follows from (3.48), (3.49) and Lemma 2 of [19].

\section{THE ZERO DIFFUSION LIMIT}

Our goal in this section will be to establish the limits (1.13) under the assumption (1.12) on the initial data for the diffusive LSW system (1.7), (1.11). First let us recall how the method of characteristics can be applied to give a representation for the solution of (1.6). For any $x, t \geq 0$ let $x(s), 0 \leq s \leq t$, be the solution of the terminal value problem,

$$
\frac{d x}{d s}=-\left[1-\left(\frac{x(s)}{L(s)}\right)^{1 / 3}\right], \quad x(t)=x .
$$

We set $F(x, t)=x(0)$, whence $F(\cdot, t)$ is a mapping on the non-negative reals with derivative

$$
\frac{\partial F(x, t)}{\partial x}=\exp \left[-\frac{1}{3} \int_{0}^{t} \frac{d s}{\left\{x(s)^{2} L(s)\right\}^{1 / 3}}\right] .
$$

The solution $c_{0}(x, t), x, t \geq 0$, of (1.6) is given in terms of the initial data $c_{0}(x), x \geq$ 0 , by the formula,

$$
\int_{x}^{\infty} c_{0}\left(x^{\prime}, t\right) d x^{\prime}=\int_{F(x, t)}^{\infty} c_{0}\left(x^{\prime}\right) d x^{\prime}, \quad x, t \geq 0 .
$$


Lemma 4.1. Let $L(\cdot)$ be a continuous function on the interval $[0, T]$ satisfying $\inf L(\cdot)>0$ and $F(x, t), x \geq 0,0 \leq t \leq T$, be the corresponding mapping derived from [4.1). Suppose $c_{0}(\cdot)$ is a nonnegative function on $(0, \infty)$ satisfying $\int_{0}^{\infty} c_{0}(x) d x<\infty$, and $c_{\varepsilon}(x, t), L_{\varepsilon}(t)$ is the solution of (1.11) with initial data $c_{0}(\cdot)$ and Dirichlet boundary condition $c_{\varepsilon}(0, t)=0$. Then if the functions $L_{\varepsilon}(\cdot)$ converge uniformly to the function $L(\cdot)$ on the interval $[0, T]$ as $\varepsilon \rightarrow 0$, one has

$$
\lim _{\varepsilon \rightarrow 0} \int_{x}^{\infty} c_{\varepsilon}\left(x^{\prime}, T\right) d x^{\prime}=\int_{F(x, T)}^{\infty} c_{0}\left(x^{\prime}\right) d x^{\prime}, \quad x \geq 0 .
$$

Proof. As in $\S 3$ we use the solution to the adjoint problem. Thus let $\mathcal{L}_{t, \varepsilon}^{*}$ be the operator,

$$
\mathcal{L}_{t, \varepsilon}^{*}=\varepsilon(1+x / \varepsilon)^{1 / 3} \frac{\partial^{2}}{\partial x^{2}}-\left[1-\left\{\frac{x}{L_{\varepsilon}(t)}\right\}^{1 / 3}\right] \frac{\partial}{\partial x},
$$

and $w_{\varepsilon}$ satisfy $\partial w_{\varepsilon} / \partial t=-\mathcal{L}_{t, \varepsilon}^{*} w_{\varepsilon}, t<T, w_{\varepsilon}(x, T)=w_{0}(x), w_{\varepsilon}(0, t)=0$. Then

$$
\int_{0}^{\infty} w_{0}(x) c_{\varepsilon}(x, T) d x=\int_{0}^{\infty} w_{\varepsilon}(x, 0) c_{0}(x) d x .
$$

For any $x_{0} \geq 0$ we take $w_{0}(x)=1, x \geq x_{0}, w_{0}(x)=0, x<x_{0}$. Evidently $w_{\varepsilon}(x, 0) \leq 1, x \geq 0$. Hence to prove the result we need to show that

$$
\begin{aligned}
\lim _{\varepsilon \rightarrow 0} w_{\varepsilon}(x, 0) & =1, \quad x>F\left(x_{0}, T\right), \\
& =0, \quad x<F\left(x_{0}, T\right) .
\end{aligned}
$$

Recall now that $w_{\varepsilon}(x, 0)=P\left(X_{\varepsilon}(T)>x_{0} ; \tau_{x, 0}>T \mid X_{\varepsilon}(0)=x\right)$, where $X_{\varepsilon}(s)$ is the solution to the stochastic equation,

$$
d X_{\varepsilon}(s)=-\left[1-\left\{\frac{X_{\varepsilon}(s)}{L_{\varepsilon}(s)}\right\}^{1 / 3}\right] d s+\sqrt{2 \varepsilon}\left(1+X_{\varepsilon}(s) / \varepsilon\right)^{1 / 6} d W(s)
$$

and $\tau_{x, t}$ is the first hitting time at the boundary for the process started at $x$ at time $t$. We first show that

$$
\lim _{\varepsilon \rightarrow 0} P\left(\tau_{x, 0}>T \mid X_{\varepsilon}(0)=x\right)=0, x<F(0, T) .
$$

By our assumptions there exists $\varepsilon_{0}>0$ and $L_{0}>0$ such that for $\varepsilon \leq \varepsilon_{0}$, inf $L_{\varepsilon}(\cdot) \geq$ $L_{0}$. Hence if $Y_{\varepsilon}(s)$ is the solution to the equation,

$$
d Y_{\varepsilon}(s)=-\left[1-\left\{\frac{Y_{\varepsilon}(s)}{L_{0}}\right\}^{1 / 3}\right] d s+\sqrt{2 \varepsilon}\left(1+Y_{\varepsilon}(s) / \varepsilon\right)^{1 / 6} d W(s),
$$

then there is the inequality,

$$
P\left(\tau_{x, t}>T \mid X_{\varepsilon}(t)=x\right) \leq P\left(\tau_{x, t}>T \mid Y_{\varepsilon}(t)=x\right), t<T .
$$

Now let $u_{\varepsilon}(x)$ be the probability that the process $Y_{\varepsilon}$ started at $x<\delta$ exits the interval $[0, \delta]$ through the boundary 0 . Then one has

$$
u_{\varepsilon}(x)=\int_{x}^{\delta} \exp \left[-\int_{0}^{z} h_{\varepsilon}\left(z^{\prime}\right) d z^{\prime}\right] d z / \int_{0}^{\delta} \exp \left[-\int_{0}^{z} h_{\varepsilon}\left(z^{\prime}\right) d z^{\prime}\right] d z,
$$

where

$$
h_{\varepsilon}(z)=\left[\left(z / L_{0}\right)^{1 / 3}-1\right] / \varepsilon[1+z / \varepsilon]^{1 / 3} .
$$


Choosing $\delta=L_{0} / 2$, it is easy to see that on any interval $\left[0, \delta^{\prime}\right]$ with $\delta^{\prime}<\delta$ the functions $u_{\varepsilon}$ converge uniformly to 1 as $\varepsilon \rightarrow 0$. Next for $0<x<\delta$ let $v_{\varepsilon}(x)$ be defined by $v_{\varepsilon}(x)=E\left[\tau_{x} \mid Y_{\varepsilon}(0)=x\right]$, where $\tau_{x}$ is the first exit time from the interval $[0, \delta]$. Then $v_{\varepsilon}$ satisfies the equation,

$$
-\varepsilon(1+x / \varepsilon)^{1 / 3} v_{\varepsilon}^{\prime \prime}(x)+\left[1-\left(x / L_{0}\right)^{1 / 3}\right] v_{\varepsilon}^{\prime}(x)=1, \quad 0<x<\delta,
$$

with Dirichlet boundary condition $v_{\varepsilon}(0)=v_{\varepsilon}(\delta)=0$. It follows from the maximum principle that

$$
v_{\varepsilon}(x) \leq x /\left[1-\left(\delta / L_{0}\right)^{1 / 3}\right], 0<x<\delta .
$$

We conclude therefore that

$$
\begin{gathered}
P\left(Y_{\varepsilon}(s) \text { hits } 0 \text { before time } t \mid Y_{\varepsilon}(0)=x\right) \\
\geq u_{\varepsilon}(x)-P\left(\tau_{x}>t \mid Y_{\varepsilon}(0)=x\right) \\
\geq u_{\varepsilon}(x)-x / t\left[1-\left(\delta / L_{0}\right)^{1 / 3}\right] .
\end{gathered}
$$

Note that if $x<<t$ then the RHS of (4.13) is positive as $\varepsilon \rightarrow 0$. Now on setting $\varepsilon=0$ in (4.12) we see that the time for the classical system to exit the interval $[0, \delta]$ is $T_{x}$, where

$$
T_{x}=\int_{0}^{x} d z /\left[1-\left(z / L_{0}\right)^{1 / 3}\right]
$$

Hence we should have that

$$
\lim _{\varepsilon \rightarrow 0} P\left(\tau_{x}>T_{x}+\eta \mid Y_{\varepsilon}(0)=x\right)=0,
$$

for any $\eta>0$. In order to prove (4.14) we first show that $\lim _{\varepsilon \rightarrow 0} v_{\varepsilon}(x)=T_{x}$. From (4.12) we have that

$$
v_{\varepsilon}^{\prime}(x)=A_{\varepsilon} \exp \left[-\int_{0}^{x} h_{\varepsilon}(z) d z\right]-\int_{0}^{x} \frac{\exp \left[-\int_{z}^{x} h_{\varepsilon}\left(z^{\prime}\right) d z^{\prime}\right] d z}{\varepsilon(1+z / \varepsilon)^{1 / 3}},
$$

where $A_{\varepsilon}$ is given by the formula

$$
A_{\varepsilon}=\int_{0<z<x^{\prime}<\delta} \frac{\exp \left[-\int_{z}^{x^{\prime}} h_{\varepsilon}\left(z^{\prime}\right) d z^{\prime}\right]}{\varepsilon(1+z / \varepsilon)^{1 / 3}} d z d x^{\prime} / \int_{0}^{\delta} \exp \left[-\int_{0}^{x^{\prime}} h_{\varepsilon}(z) d z\right] d x^{\prime} .
$$

Letting $g(z)=1-\left(z / L_{0}\right)^{1 / 3}$ we have that

$$
\begin{gathered}
-\int_{0}^{x} \frac{\exp \left[-\int_{z}^{x} h_{\varepsilon}\left(z^{\prime}\right) d z^{\prime}\right]}{\varepsilon(1+z / \varepsilon)^{1 / 3}} d z=\int_{0}^{x} \frac{h_{\varepsilon}(z)}{g(z)} \exp \left[-\int_{z}^{x} h_{\varepsilon}\left(z^{\prime}\right) d z^{\prime}\right] d z \\
=\frac{1}{g(x)}-\frac{1}{g(0)} \exp \left[-\int_{0}^{x} h_{\varepsilon}\left(z^{\prime}\right) d z^{\prime}\right]+\int_{0}^{x} \frac{g^{\prime}(z)}{g(z)^{2}} \exp \left[-\int_{z}^{x} h_{\varepsilon}\left(z^{\prime}\right) d z^{\prime}\right] d z .
\end{gathered}
$$

Similarly we have that

$$
\begin{gathered}
A_{\varepsilon}=\frac{1}{g(0)}-\int_{0}^{\delta} \frac{d x^{\prime}}{g\left(x^{\prime}\right)} / \int_{0}^{\delta} \exp \left[-\int_{0}^{x^{\prime}} h_{\varepsilon}\left(z^{\prime}\right) d z^{\prime}\right] d x^{\prime} \\
-\int_{0<z<x^{\prime}<\delta} \frac{g^{\prime}(z)}{g(z)^{2}} \exp \left[-\int_{z}^{x^{\prime}} h_{\varepsilon}\left(z^{\prime}\right) d z^{\prime}\right] d z d x^{\prime} / \int_{0}^{\delta} \exp \left[-\int_{0}^{x^{\prime}} h_{\varepsilon}\left(z^{\prime}\right) d z^{\prime}\right] d x^{\prime} .
\end{gathered}
$$


We conclude that

$$
\begin{aligned}
v_{\varepsilon}^{\prime}(x) & =\frac{1}{g(x)}-\exp \left[-\int_{0}^{x} h_{\varepsilon}(z) d z\right] \int_{0}^{\delta} \frac{d x^{\prime}}{g\left(x^{\prime}\right)} / \int_{0}^{\delta} \exp \left[-\int_{0}^{x^{\prime}} h_{\varepsilon}(z) d z\right] d x^{\prime} \\
& +\int_{0}^{\delta} \frac{g^{\prime}(z)}{g(z)^{2}} \exp \left[-\int_{z}^{x} h_{\varepsilon}\left(z^{\prime}\right) d z^{\prime}\right]\{H(x-z) \\
& \left.-\int_{z}^{\delta} \exp \left[-\int_{0}^{x^{\prime}} h_{\varepsilon}\left(z^{\prime}\right) d z^{\prime}\right] d x^{\prime} / \int_{0}^{\delta} \exp \left[-\int_{0}^{x^{\prime}} h_{\varepsilon}\left(z^{\prime}\right) d z^{\prime}\right] d x^{\prime}\right\} d z
\end{aligned}
$$

where $H(y)$ is the Heaviside function, $H(y)=1, y>0, H(y)=0, y<0$. It follows easily from the previous expression that $\lim _{\varepsilon \rightarrow 0} v_{\varepsilon}^{\prime}(x)=1 / g(x)$ uniformly in any interval $\left[0, \delta^{\prime}\right]$ with $\delta^{\prime}<\delta$. Hence $\lim _{\varepsilon \rightarrow 0} v_{\varepsilon}(x)=T_{x}$ for $0 \leq x<\delta$.

We can in a similar way estimate $w_{\varepsilon}(x)=E\left[\tau_{x}^{2} \mid Y_{\varepsilon}(0)=x\right]$. In fact $w_{\varepsilon}$ satisfies the equation,

$$
-\varepsilon(1+x / \varepsilon)^{1 / 3} w_{\varepsilon}^{\prime \prime}(x)+\left[1-\left(x / L_{0}\right)^{1 / 3}\right] w_{\varepsilon}^{\prime}(x)=2 v_{\varepsilon}(x), \quad 0<x<\delta,
$$

with Dirichlet boundary condition $w_{\varepsilon}(0)=w_{\varepsilon}(\delta)=0$. Proceeding as in the previous paragraph we see that $\lim _{\varepsilon \rightarrow 0} w_{\varepsilon}^{\prime}(x)=2 T_{x} / g(x)$ uniformly in any interval $\left[0, \delta^{\prime}\right]$ with $\delta^{\prime}<\delta$. It follows that $\lim _{\varepsilon \rightarrow 0} w_{\varepsilon}(x)=T_{x}^{2}$ for $0<x<\delta$. Finally we conclude from the Chebyshev inequality that (4.14) holds.

From (4.10) and (4.14) we see that for $x$ satisfying $0<x<L_{0} / 2$ then (4.8) holds provided $T>T_{x}$. We show now that it holds for all $x<F(0, T)$. To do this let $\bar{\tau}_{x, t}$ be the first hitting time for the process $X_{\varepsilon}(s)$ with $X_{\varepsilon}(t)=x>L_{0} / 4$ to hit $L_{0} / 4$. Putting $\bar{\tau}=\tau_{x, 0}$ we see as in Lemma 3.2 that

$$
\begin{aligned}
E\left[X_{\varepsilon}\left(t^{\prime} \wedge \bar{\tau}\right)^{2}\right] & =x^{2}+2 E\left[\int_{0}^{t^{\prime} \wedge \bar{\tau}} X_{\varepsilon}(s)\left[\left\{\frac{X_{\varepsilon}(s)}{L_{\varepsilon}(s)}\right\}^{1 / 3}-1\right] d s\right] \\
& +2 \varepsilon E\left[\int_{0}^{t^{\prime} \wedge \bar{\tau}}\left(1+X_{\varepsilon}(s) / \varepsilon\right)^{1 / 3} d s\right] .
\end{aligned}
$$

It follows that there is a constant $C\left(L_{0}\right)$ depending only on $L_{0}$ such that

$$
E\left[X_{\varepsilon}\left(t^{\prime} \wedge \bar{\tau}\right)^{2}\right] \leq x^{2}+C\left(L_{0}\right) E\left[\int_{0}^{t^{\prime} \wedge \bar{\tau}} X_{\varepsilon}(s \wedge \bar{\tau})^{2} d s\right], \quad 0 \leq t^{\prime} \leq T .
$$

Gronwall's inequality therefore yields,

$$
E\left[X_{\varepsilon}\left(t^{\prime} \wedge \bar{\tau}\right)^{2}\right] \leq x^{2} \exp \left[C\left(L_{0}\right) t^{\prime}\right], \quad 0 \leq t^{\prime} \leq T .
$$

Next, applying Ito's lemma to $\left[X_{\varepsilon}\left(t^{\prime}\right)-X_{0}\left(t^{\prime}\right)\right]^{2}$ we see from (4.7) that

$$
\begin{aligned}
{\left[X_{\varepsilon}\left(t^{\prime}\right)-X_{0}\left(t^{\prime}\right)\right]^{2} } & =2 \int_{0}^{t^{\prime}}\left[X_{\varepsilon}(s)-X_{0}(s)\right]\left[\left\{\frac{X_{\varepsilon}(s)}{L_{\varepsilon}(s)}\right\}^{1 / 3}-\left\{\frac{X_{0}(s)}{L_{0}(s)}\right\}^{1 / 3}\right] d s \\
& +2 \sqrt{2 \varepsilon} \int_{0}^{t^{\prime}}\left[X_{\varepsilon}(s)-X_{0}(s)\right]\left[1+X_{\varepsilon}(s) / \varepsilon\right]^{1 / 6} d W(s)+2 \varepsilon \int_{0}^{t^{\prime}}\left[1+X_{\varepsilon}(s) / \varepsilon\right]^{1 / 3} d s .
\end{aligned}
$$

With $\bar{\tau}$ as in (4.16) we then have that 


$$
\begin{gathered}
E\left\{\left[X_{\varepsilon}\left(t^{\prime} \wedge \bar{\tau}\right)-X_{0}\left(t^{\prime} \wedge \bar{\tau}\right)\right]^{2}\right\} \leq C\left(L_{0}\right)\left\{\left[\sup _{0 \leq s \leq T}\left|L_{\varepsilon}(s)-L_{0}(s)\right|+\varepsilon^{2 / 3}\right] x^{2} \exp \left[C\left(L_{0}\right) t^{\prime}\right]\right. \\
+\int_{0}^{t^{\prime} \wedge \bar{\tau}} E\left\{\left[X_{\varepsilon}(s \wedge \bar{\tau})-X_{0}(s \wedge \bar{\tau})\right]^{2} d s\right\}, \quad 0 \leq t^{\prime} \leq T \wedge T_{x},
\end{gathered}
$$

for a constant $C\left(L_{0}\right)$ depending only on $L_{0}$, where $T_{x}$ is defined by $X_{0}\left(T_{x}\right)=0$.

Again from the Ito lemma we have that

$$
X_{\varepsilon}\left(t^{\prime}\right)-X_{0}\left(t^{\prime}\right)=\int_{0}^{t^{\prime}}\left\{\frac{X_{\varepsilon}(s)}{L_{\varepsilon}(s)}\right\}^{1 / 3}-\left\{\frac{X_{0}(s)}{L_{0}(s)}\right\}^{1 / 3} d s+\sqrt{2 \varepsilon} \int_{0}^{t^{\prime}}\left[1+X_{\varepsilon}(s) / \varepsilon\right]^{1 / 6} d W(s) .
$$

Now by the Kolmogorov inequality we have that for $\eta>0$,

$$
P\left(\sup _{0 \leq t^{\prime} \leq T}\left|\sqrt{2 \varepsilon} \int_{0}^{t^{\prime} \wedge \bar{\tau}}\left[1+X_{\varepsilon}(s) / \varepsilon\right]^{1 / 6} d W(s)\right|>\eta / 2\right) \leq \frac{8 \varepsilon}{\eta^{2}} E\left[\int_{0}^{T \wedge \bar{\tau}}\left[1+X_{\varepsilon}(s) / \varepsilon\right]^{1 / 3} d s\right] .
$$

We also have that

$$
\begin{array}{r}
P\left(\sup _{0 \leq t^{\prime} \leq T \wedge T_{x}}\left|\int_{0}^{t^{\prime} \wedge \bar{\tau}}\left\{\frac{X_{\varepsilon}(s)}{L_{\varepsilon}(s)}\right\}^{1 / 3}-\left\{\frac{X_{0}(s)}{L_{\varepsilon}(s)}\right\}^{1 / 3} d s\right|>\eta / 4\right) \\
\leq \frac{C\left(L_{0}\right)}{\eta} \sqrt{T} E\left[\int_{0}^{T \wedge T_{x} \wedge \bar{\tau}}\left\{X_{\varepsilon}(s)-X_{0}(s)\right\}^{2} d s\right]^{1 / 2},
\end{array}
$$

for a constant $C\left(L_{0}\right)$ depending only on $L_{0}$. It follows then from (4.16), (4.17), (4.18) that for any $\eta>0$,

$$
\lim _{\varepsilon \rightarrow 0} P\left(\sup _{0 \leq t^{\prime} \leq T \wedge T_{x} \wedge \bar{\tau}}\left|X_{\varepsilon}\left(t^{\prime}\right)-X_{0}\left(t^{\prime}\right)\right|>\eta\right)=0 .
$$

We finish the proof of (4.8). For $x<F(0, T)$ note that $T_{x}<T$. We take $\eta$ in (4.19) so that $\eta<L_{0} / 4$ and consider the event $\left\{\left|X_{\varepsilon}\left(T_{x} \wedge \bar{\tau}\right)-X_{0}\left(T_{x} \wedge \bar{\tau}\right)\right|<\eta\right\}$. We evidently must have $\bar{\tau}<T_{x}$ for this event. Since then $\left|L_{0} / 4-X_{0}(\bar{\tau})\right|<\eta$ the stopping time $\bar{\tau}$ must be close to the time required for the classical trajectory to go from $x$ to $L_{0} / 4$. Now by starting the diffusion $X_{\varepsilon}$ at time $t=\bar{\tau}$ and using (4.10), (4.14) we conclude that (4.8) holds.

To complete the proof of the lemma we need to show (4.6). For $x>F(0, T)$ one has $T_{x}>T$ whence the event $\left\{\left|X_{\varepsilon}\left(T_{x} \wedge \bar{\tau}\right)-X_{0}\left(T_{x} \wedge \bar{\tau}\right)\right|<\eta\right\}$ has high probability. If $\bar{\tau}>T$ then $\tau_{x, 0}>T$ so we consider the situation $\bar{\tau}<T$. Since $\left|L_{0} / 4-X_{0}(\bar{\tau})\right|<\eta$ in this case, the stopping time $\bar{\tau}$ must again be close to the time required for the classical trajectory to go from $x$ to $L_{0} / 4$. Using our previous argument we can estimate the time taken for the diffusion $X_{\varepsilon}$ started at time $t=\bar{\tau}$ at $L_{0} / 4$ to hit 0 . For $\varepsilon$ small this is close to the time for the classical trajectory, whence $\tau_{x, 0}>T$ with high probability. We conclude that

$$
\lim _{\varepsilon \rightarrow 0} P\left(\tau_{x, 0}>T \mid X_{\varepsilon}(0)=x\right)=1, \quad x>F(0, T) .
$$

This completes the proof of (4.6) for $x_{0}=0$. The argument for $x_{0}>0$ is similar.

Lemma 4.2. Let $c_{\varepsilon}(x, t), L_{\varepsilon}(t), t \geq 0$, be a solution of the system (1.7), (1.11) with initial data $c_{0}(x)$ satisfying $c_{0}(x) \geq 0, x \geq 0, \int_{0}^{\infty}(1+x) c_{0}(x) d x<\infty$, 
$\int_{0}^{\infty} x c_{0}(x) d x=1$. Then for any $\varepsilon_{0}, T>0$ the set of functions $L_{\varepsilon}(\cdot), 0<\varepsilon \leq \varepsilon_{0}$, on the interval $[0, T]$ is an equicontinuous family.

Proof. Let us first consider the classical LSW system (1.6), (1.7) and let $\Lambda(t), t \geq 0$, be the mean particle volume as in (1.14). Then one has by Jensen's inequality that $L(t) \leq \Lambda(t)$ and it is also easy to see that $\Lambda(t)$ is an increasing function. For $x \geq 0$ let $w_{0}(x)$ be defined by

$$
w_{0}(x)=\int_{x}^{\infty} c_{0}\left(x^{\prime}\right) d x^{\prime} .
$$

From (4.3) we have that $\Lambda(t)=1 / w_{0}(F(0, t))$ and one can further see that $F(0, t)<$ $t$. Noting that $\Lambda(0)$ is given by

$$
\Lambda(0)=\int_{0}^{\infty} x c_{0}(x) d x / \int_{0}^{\infty} c_{0}(x) d x
$$

we see that

$$
\int_{\Lambda(0) / 2}^{\infty} x c_{0}(x) d x \geq \frac{1}{2} \int_{0}^{\infty} x c_{0}(x) d x=\frac{1}{2}
$$

whence $w_{0}(\Lambda(0) / 2)>0$. Hence $\Lambda(\cdot)$ is bounded above in the interval $[0, \Lambda(0) / 4]$, and a-fortiori $L(\cdot)$ is bounded above in $[0, \Lambda(0) / 4]$. Next we obtain a lower bound on $L(\cdot)$ in this interval. To do this we use the formula (3.1) for $L(t)$ which, if we denote by $w(x, t)$ the LHS of (4.3), is the same as

$$
L(t)^{1 / 3}=\frac{1}{3} \int_{0}^{\infty} x^{-2 / 3} w(x, t) d x / w(0, t) .
$$

Since $F(x, t)<x+t$ we have that

$$
\begin{aligned}
\frac{1}{3} \int_{0}^{\infty} x^{-2 / 3} w(x, t) d x & \geq \frac{1}{3} \int_{0}^{\infty} x^{-2 / 3} w_{0}(x+t) d x \\
& \geq a^{1 / 3} w_{0}(a+t), \quad a>0, \quad t>0 .
\end{aligned}
$$

We have then that

$$
L(t)^{1 / 3} \geq \Lambda(t) a^{1 / 3} w_{0}(a+t), \quad t>0, \quad a>0 .
$$

If in this last inequality we set $a=\Lambda(0) / 4$ we see that $L(\cdot)$ is bounded below on the interval $[0, \Lambda(0) / 4]$ by a positive constant. This argument can be easily extended, using the fact that $\Lambda(\cdot)$ is an increasing function, to conclude that $\Lambda(\cdot)$ is bounded above on any interval $[0, T]$ and $L(\cdot)$ is bounded below by a positive constant on the interval.

The boundedness below of $L(\cdot)$ on $[0, T]$ implies the continuity of the functions $\Lambda(\cdot), L(\cdot)$. To see this note that for $\delta>0$,

$$
0<\Lambda(t)^{-1}-\Lambda(t+\delta)^{-1}<w_{0}(F(0, t))-w_{0}(F(\delta, t)) .
$$

In view of (4.2) we have that $0<F(\delta, t)-F(0, t)<\delta$. The continuity of $\Lambda(\cdot)$ follows now from the continuity of $w_{0}(\cdot)$. To show continuity of $L(\cdot)$ we note that since

$$
\int_{0}^{\infty} w_{0}(F(x, t)) d x=1,
$$

there exists for any $\eta>0$ an $x_{\eta}>0$ such that

$$
\int_{x_{\eta}}^{\infty} x^{-2 / 3} w_{0}(F(x, t)) d x<\eta
$$


We consider the integral

$$
\begin{gathered}
\int_{0}^{2 x_{\eta}} x^{-2 / 3}\left[w_{0}(F(x, t))-w_{0}(F(x, t+\delta))\right] d x= \\
\int_{0}^{2 x_{\eta}} x^{-2 / 3}\left[w_{0}(F(x, t))-w_{0}(F(g(x, t, \delta), t))\right] d x,
\end{gathered}
$$

where

$$
|g(x, t, \delta)-x| \leq \delta\left[1+\left(x / L_{0}\right)^{1 / 3}\right] .
$$

Using the continuity of $w_{0}(\cdot)$ and (4.2) we conclude that $\delta$ can be chosen sufficiently small that the integral in (4.21) is bounded in absolute value by $\eta$. Hence from (4.20) we have that

$$
\left|\int_{0}^{\infty} x^{-2 / 3}\left[w_{0}(F(x, t))-w_{0}(F(x, t+\delta))\right] d x\right|<3 \eta
$$

This last inequality and the continuity of $\Lambda(\cdot)$ implies the continuity of $L(\cdot)$.

Next we wish to apply the previous argument to the system (1.7), (1.11). Let $\Lambda_{\varepsilon}(t)$ be as in (1.14), whence it follows that $\Lambda_{\varepsilon}(t)$ is an increasing function of $t$ and $\Lambda_{\varepsilon}(0)=\Lambda(0)$. From (4.5) we have that

$$
\Lambda_{\varepsilon}(T)^{-1}=\int_{0}^{\infty} w_{\varepsilon}(x, 0) c_{0}(x) d x,
$$

where $w_{\varepsilon}(x, T)=1$ and $w_{\varepsilon}$ is a solution to the adjoint equation as in Lemma 4.1. It is easy to see from (4.7) that

$$
w_{\varepsilon}(x, 0) \geq P\left(\tau_{x, 0}>T \mid X_{\varepsilon}(0)=x\right),
$$

where $X_{\varepsilon}(s)$ is the solution to the stochastic equation,

$$
d X_{\varepsilon}(s)=-d s+\sqrt{2 \varepsilon}\left(1+X_{\varepsilon}(s) / \varepsilon\right)^{1 / 6} d W(s) .
$$

We shall show that there is a positive constant $\gamma\left(T, \varepsilon_{0}\right)$ depending only on $T$ and $\varepsilon_{0}$ such that

$$
P\left(\tau_{x, 0}>T \mid X_{\varepsilon}(0)=x\right) \geq \gamma\left(T, \varepsilon_{0}\right), \quad x>2 T,
$$

provided $0<\varepsilon \leq \varepsilon_{0}$. To see this we argue as in Lemma 4.1. Thus from Ito's lemma applied to (4.23) we have that

$E\left[X_{\varepsilon}\left(t \wedge \tau_{x, 0}\right)^{2}\right]=x^{2}-2 E\left[\int_{0}^{t \wedge \tau_{x, 0}} X_{\varepsilon}(s) d s\right]+2 \varepsilon E\left[\int_{0}^{t \wedge \tau_{x, 0}}\left[1+X_{\varepsilon}(s) / \varepsilon\right]^{1 / 3} d s\right]$.

On using the inequality,

$$
(1+z)^{1 / 3} \leq 1+z^{1 / 3} \leq 1+\varepsilon^{-1 / 3}+\varepsilon^{5 / 3} z^{2},
$$

we see then that

$$
E\left[X_{\varepsilon}\left(t \wedge \tau_{x, 0}\right)^{2}\right] \leq x^{2}+2 t\left(\varepsilon+\varepsilon^{2 / 3}\right)+2 \varepsilon^{2 / 3} E\left[\int_{0}^{t \wedge \tau_{x, 0}} X_{\varepsilon}(s)^{2} d s\right] .
$$

It follows from the Gronwall inequality that

$$
E\left[X_{\varepsilon}\left(t \wedge \tau_{x, 0}\right)^{2}\right] \leq\left[x^{2}+2 t\left(\varepsilon+\varepsilon^{2 / 3}\right)\right] \exp \left[2 \varepsilon^{2 / 3} t\right] .
$$

We also have from (4.23) that

$$
X_{\varepsilon}\left(t \wedge \tau_{x, 0}\right)-x+t=\sqrt{2 \varepsilon} \int_{0}^{t \wedge \tau_{x, 0}}\left[1+X_{\varepsilon}(s) / \varepsilon\right]^{1 / 6} d W(s)
$$


whence one has that

$$
\eta^{2} P\left(\sup _{0 \leq t \leq T}\left|X_{\varepsilon}\left(t \wedge \tau_{x, 0}\right)-x+t\right|>\eta\right) \leq 2 \varepsilon E\left[\int_{0}^{T \wedge \tau_{x, 0}}\left[1+X_{\varepsilon}(s) / \varepsilon\right]^{1 / 3} d s\right] .
$$

Using now (4.25) and the Hölder inequality we conclude that

$\eta^{2} P\left(\sup _{0 \leq t \leq T}\left|X_{\varepsilon}\left(t \wedge \tau_{x, 0}\right)-x+t\right|>\eta\right) \leq 4 T \varepsilon^{2 / 3}\left\{\varepsilon^{2}+\left[x^{2}+2 T\left(\varepsilon+\varepsilon^{2 / 3}\right)\right] \exp \left[2 \varepsilon^{2 / 3} T\right]\right\}^{1 / 6}$.

Choosing $\eta=x / 2$ in (4.26) we see that there is an $x_{T} \geq 2 T$ such that the RHS of (4.26) divided by $\eta^{2}$ does not exceed $1 / 2$ provided $x \geq x_{T}, 0<\varepsilon \leq \varepsilon_{0}$. Furthermore there exists $\varepsilon_{T}>0$ such that we may take $x_{T}=2 T$ if $\varepsilon$ is in the region $0<\varepsilon \leq \varepsilon_{T}$. We conclude that

$$
\begin{aligned}
P\left(\tau_{x, 0}>T \mid X_{\varepsilon}(0)=x\right) & \geq 1 / 2, x>2 T, 0<\varepsilon \leq \varepsilon_{T}, \\
& \geq 1 / 2, x>x_{T}, 0<\varepsilon \leq \varepsilon_{0} .
\end{aligned}
$$

To complete the proof of (4.24) then we need to show that

$$
P\left(\tau_{x, 0}>T \mid X_{\varepsilon}(0)=x\right) \geq \gamma\left(T, \varepsilon_{0}\right), 2 T<x<x_{T}, \varepsilon_{T}<\varepsilon<\varepsilon_{0} .
$$

This can be demonstrated using perturbation theory. Choose an integer $N$ and points $x_{j}=2 j T / N, j=0,1, \ldots, N+1$. Now define $p_{j}, j=1, \ldots, N$ by

$$
\begin{aligned}
& p_{j}=\inf _{\varepsilon_{T}<\varepsilon<\varepsilon_{0}}\left\{\text { Probability } X_{\varepsilon}(t) \text { with } X_{\varepsilon}(0)=x_{j}\right. \\
& \text { exits the interval } \left.\left[x_{j-1}, x_{j+1}\right] \text { in time greater than } T / N\right\} \text {. }
\end{aligned}
$$

Then the LHS of (4.28) is bounded below by $p_{1} \cdots p_{N}$. To estimate the $p_{j}$ we choose $N$ large enough so that the Dirichlet Green's function for the interval $\left[x_{j-1}, x_{j+1}\right]$ can be expanded in a converging perturbation series as in Lemma 3.1. The integer $N$ can be chosen dependent only on $T$ because $\varepsilon_{T}<\varepsilon<\varepsilon_{0}$. One can thus show that the solution of the diffusion equation corresponding to (4.23) with initial data 1 and Dirichlet boundary conditions is bounded below by a constant depending only on $T$ at $x=x_{j}, t=T / N$. Hence we obtain a lower bound on $p_{j}$ and thus have established (4.28).

We have now from (4.22), (4.24) that

$$
\Lambda_{\varepsilon}(T) \leq 1 / \gamma\left(T, \varepsilon_{0}\right) \int_{2 T}^{\infty} c_{0}(x) d x,
$$

whence $\Lambda_{\varepsilon}(T)$ is uniformly bounded above for $0<\varepsilon \leq \varepsilon_{0}$ provided $T \leq \Lambda(0) / 4$. Arguing as before we can obtain a uniform lower bound on $L_{\varepsilon}(t), \quad 0 \leq t \leq \Lambda(0) / 4$. To do this we write

$$
\int_{0}^{\infty} x^{1 / 3} c_{\varepsilon}(x, t) d x=\frac{1}{3} \int_{0}^{\infty} x^{-2 / 3} \int_{x}^{\infty} c_{\varepsilon}\left(x^{\prime}, t\right) d x^{\prime},
$$

whence for any $a>0$,

$$
L_{\varepsilon}(t)^{1 / 3} \geq \Lambda_{\varepsilon}(t) a^{1 / 3} \int_{a}^{\infty} c_{\varepsilon}\left(x^{\prime}, t\right) d x^{\prime} .
$$


Now in the same way as we obtained (4.29) we see from this that there is a positive constant $K(\Lambda(0))$ depending only on $\Lambda(0)$ such that

$$
L_{\varepsilon}(t)^{1 / 3} \geq K(\Lambda(0)) \int_{\Lambda(0) / 2}^{\infty} c_{0}(x) d x, 0 \leq t \leq \Lambda(0) / 4,0<\varepsilon \leq \varepsilon_{0} .
$$

We have shown that the functions $\Lambda_{\varepsilon}(\cdot), L_{\varepsilon}(\cdot), 0<\varepsilon \leq \varepsilon_{0}$, are uniformly bounded above and away from zero in the interval $0 \leq t \leq \Lambda(0) / 4$. Next we show that they are equicontinuous. To do this we use a formula analogous to (4.22). Thus for $\delta>0$ we write

$$
\Lambda_{\varepsilon}(T)-\Lambda_{\varepsilon}(T+\delta)^{-1}=\int_{0}^{\infty} w_{\varepsilon}(x, 0) c_{0}(x) d x,
$$

where $w_{\varepsilon}(x, t)$ is a solution to the adjoint equation as in Lemma 4.1 with terminal data specified at $t=T$. The terminal data is given by

$$
w_{\varepsilon}(x, T)=1-P\left(X_{\varepsilon}(s)>0, T<s<T+\delta \mid X_{\varepsilon}(T)=x\right),
$$

where $X_{\varepsilon}(s)$ satisfies the stochastic equation (4.7). It is easy to see that

$$
w_{\varepsilon}(x, T) \leq 1-P\left(X_{\varepsilon}(s)>0,0<s<\delta \mid X_{\varepsilon}(0)=x\right),
$$

where $X_{\varepsilon}(s)$ satisfies the stochastic equation (4.23). We can use (4.26) then to estimate the RHS of (4.32). Thus on setting $T=\delta$ and $\eta=\sqrt{\delta}+x / 2$ in (4.26) we see from (4.32) that $w_{\varepsilon}(x, T)$ satisfies the inequalities,

$$
\begin{aligned}
& w_{\varepsilon}(x, T) \leq 1, \quad 0<x<4 \sqrt{\delta}, \\
& w_{\varepsilon}(x, T) \leq C\left(\varepsilon_{0}\right) \delta\left[1+x^{1 / 3}\right] / x^{2}, x>4 \sqrt{\delta},
\end{aligned}
$$

where $C\left(\varepsilon_{0}\right)$ is a constant depending only on $\varepsilon_{0}$.

We shall show that if $w_{\varepsilon}(x, T)$ satisfies (4.33) then the RHS of (4.31) is small for small $\delta$, uniformly in $\varepsilon, 0<\varepsilon \leq \varepsilon_{0}$. To see this we first consider the case where $0<\varepsilon<\delta^{1 / 4}$. Let $X_{\varepsilon}(s)$ satisfy (4.7) and $Y_{\varepsilon}(s)$ the corresponding deterministic equation,

$$
d Y_{\varepsilon}(s)=-\left[1-\left\{\frac{Y_{\varepsilon}(s)}{L_{\varepsilon}(s)}\right\}^{1 / 3}\right] d s, \quad 0<s<T .
$$

We obtain an estimate on the size of the difference $X_{\varepsilon}(T)-Y_{\varepsilon}(T)$ when $X_{\varepsilon}(0)=$ $Y_{\varepsilon}(0)=x$, which is similar to (4.26). Letting $\tau_{x}$ be the first exit time from the interval $(0, \infty)$ for the process $X_{\varepsilon}(s)$ started at $x$ at $s=0$ we have as in the derivation of (4.25) that

$$
E\left[X_{\varepsilon}\left(t \wedge \tau_{x, 0}\right)^{2}\right] \leq x^{2}+2 t\left(\varepsilon+\varepsilon^{2 / 3}\right)+2 t / L_{0}^{1 / 3}+\left(2 \varepsilon^{2 / 3}+2 / L_{0}^{1 / 3}\right) E\left[\int_{0}^{t \wedge \tau_{x}} X_{\varepsilon}(s)^{2} d s\right],
$$

where $L_{0}$ is a lower bound on $L_{\varepsilon}(\cdot)$. We conclude that

$$
E\left[X_{\varepsilon}\left(t \wedge \tau_{x, 0}\right)^{2}\right] \leq\left[x^{2}+2 t\left(\varepsilon+\varepsilon^{2 / 3}+1 / L_{0}^{1 / 3}\right)\right] \exp \left[2\left(\varepsilon^{2 / 3}+1 / L_{0}^{1 / 3}\right) t\right] .
$$

From (4.7), (4.34) we have that

$$
\begin{gathered}
E\left\{\left[X_{\varepsilon}\left(t \wedge \tau_{x}\right)-Y_{\varepsilon}\left(t \wedge \tau_{x}\right)\right]^{2}\right\}=2 E\left\{\int_{0}^{t \wedge \tau_{x}}\left[X_{\varepsilon}(s)-Y_{\varepsilon}(s)\right]\left[X_{\varepsilon}(s)^{1 / 3}-Y_{\varepsilon}(s)^{1 / 3}\right] / L_{\varepsilon}(s)^{1 / 3} d s\right\} \\
+2 \varepsilon E\left\{\int_{0}^{t \wedge \tau_{x}}\left[1+X_{\varepsilon}(s) / \varepsilon\right]^{1 / 3} d s\right\},
\end{gathered}
$$


provided $Y_{\varepsilon}(s)>0,0 \leq s \leq t$. Setting $y(t)$ to be the LHS of (4.36) we see that

$$
\begin{aligned}
|d y / d t| & \leq 2 y(t) / L_{0}^{1 / 3} Y_{\varepsilon}(t)^{2 / 3}+2 \varepsilon E\left\{\left[1+X_{\varepsilon}\left(t \wedge \tau_{x}\right) / \varepsilon\right]^{1 / 3}\right\} \\
& \leq 2 y(t) / L_{0}^{1 / 3} Y_{\varepsilon}(t)^{2 / 3}+2\left(\varepsilon+\varepsilon^{2 / 3}\right)+2 \varepsilon^{2 / 3} E\left[X_{\varepsilon}\left(t \wedge \tau_{x}\right)^{2}\right] .
\end{aligned}
$$

Integrating this last inequality we conclude that

$$
\begin{aligned}
& E\left\{\left[X_{\varepsilon}\left(t \wedge \tau_{x}\right)-Y_{\varepsilon}\left(t \wedge \tau_{x}\right)\right]^{2}\right\} \leq \\
& \int_{0}^{t} d t^{\prime}\left\{2\left(\varepsilon+\varepsilon^{2 / 3}\right)+2 \varepsilon^{2 / 3} E\left[X_{\varepsilon}\left(t^{\prime} \wedge \tau_{x}\right)^{2}\right]\right\} \exp \left[\int_{t^{\prime}}^{t} 2 / L_{0}^{1 / 3} Y_{\varepsilon}(s)^{2 / 3} d s\right] .
\end{aligned}
$$

Using (4.35) we see from (4.37) that $X_{\varepsilon}\left(t \wedge \tau_{x}\right)$ differs from $Y_{\varepsilon}\left(t \wedge \tau_{x}\right)$ by $O\left(\varepsilon^{1 / 3}\right)$.

Recall now that the function $w_{\varepsilon}(x, 0)$ in (4.31) is given by the formula,

$$
w_{\varepsilon}(x, 0)=E\left[w_{\varepsilon}\left(X_{\varepsilon}(T), T\right) ; \tau_{x}>T \mid X_{\varepsilon}(0)=x\right],
$$

where $w_{\varepsilon}(x, T)$ satisfies (4.33). It is easy to see that the RHS of (4.31) is small for small $\delta$ uniformly in $\varepsilon, 0<\varepsilon<\varepsilon_{0}$, if we replace $X_{\varepsilon}(T)$ in (4.38) by $Y_{\varepsilon}(T)$. In fact let $a_{\varepsilon}$ have the property that if $Y_{\varepsilon}(0)=a_{\varepsilon}$ then $Y_{\varepsilon}(T)=0$, whence $w_{\varepsilon}(x, 0)=$ $0, x<a_{\varepsilon}$. Now from (4.2) it follows that if $Y_{\varepsilon}(0)>a_{\varepsilon}+\delta^{1 / 24}$ then $Y_{\varepsilon}(T)>\delta^{1 / 24}$. We conclude therefore that $w_{\varepsilon}(x, 0)$ satisfies the inequalities,

$$
\begin{aligned}
& w_{\varepsilon}(x, 0)=0, x<a_{\varepsilon} ; \quad w_{\varepsilon}(x, 0) \leq 1, a_{\varepsilon}<x<a_{\varepsilon}+\delta^{1 / 24}, \\
& w_{\varepsilon}(x, 0) \leq K \delta^{11 / 12}, \quad x>a_{\varepsilon}+\delta^{1 / 24}
\end{aligned}
$$

for a constant $K$ depending only on $\varepsilon_{0}$. Since $c_{0}(\cdot)$ is an $L^{1}$ function 4.39) implies that the RHS of (4.31) is small for small $\delta$, uniformly in $\varepsilon, 0<\varepsilon<\varepsilon_{0}$.

We can extend the argument in the previous paragraph to estimate the actual function $w_{\varepsilon}(x, 0)$ of (4.38) by using (4.37). In fact one sees using the Chebyshev inequality and $w_{\varepsilon}(\cdot, T) \leq 1$ that

$$
w_{\varepsilon}(x, 0) \leq K \delta^{1 / 12}, \quad x>a_{\varepsilon}+\delta^{1 / 24} .
$$

Here we are using the assumption $\varepsilon<\delta^{1 / 4}$. Next we show that $w_{\varepsilon}(x, 0)$ is small if $x<a_{\varepsilon}-\delta^{1 / 24}$. To see this first note that for such an $x$ then $Y_{\varepsilon}(t)$ with $Y_{\varepsilon}(0)=x$ satisfies $Y_{\varepsilon}(t)=0$ for some $t=t_{\varepsilon}$ satisfying $t_{\varepsilon}<T-\delta^{1 / 24}$. Now from (4.37) one has then that $X_{\varepsilon}\left(t_{\varepsilon} \wedge \tau_{x}\right)<O\left(\delta^{1 / 12}\right)$ with high probability. We need to show that this implies that $\tau_{x}<T$ with high probability. We consider the probability that the diffusion $X_{\varepsilon}(t)$ with $X_{\varepsilon}\left(t_{\varepsilon}\right)=x$ hits 0 before time $t_{\varepsilon}+\delta^{1 / 24}$. This is greater than the probability of the diffusion $Y_{\varepsilon}(s)$ defined by (4.9) with $Y_{\varepsilon}(0)=x$ hitting 0 before time $\delta^{1 / 24}$. Let $I_{\delta}$ be the interval $\left[0, \delta^{3 / 48}\right]$, and $u_{\varepsilon}(x)$ be the probability that $Y_{\varepsilon}(s)$ with $Y_{\varepsilon}(0)=x$ exits $I_{\delta}$ through 0 . As previously $u_{\varepsilon}(x)$ is given by the formula,

$$
u_{\varepsilon}(x)=\int_{x}^{\delta^{3 / 48}} \exp \left[-\int_{0}^{z} h_{\varepsilon}\left(z^{\prime}\right) d z^{\prime}\right] d z / \int_{0}^{\delta^{3 / 48}} \exp \left[-\int_{0}^{z} h_{\varepsilon}\left(z^{\prime}\right) d z^{\prime}\right] d z,
$$

where $h_{\varepsilon}(z)$ is defined by (4.11). Next let $v_{\varepsilon}(x)$ be the expected time for the diffusion $Y_{\varepsilon}(s)$ with $Y_{\varepsilon}(0)=x$ to exit $I_{\delta}$. Then there is the inequality,

$1-P\left(Y_{\varepsilon}(s)\right.$ hits 0 before time $\left.\delta^{1 / 24} \mid Y_{\varepsilon}(0)=x\right) \leq 1-u_{\varepsilon}(x)+\delta^{-1 / 24} v_{\varepsilon}(x), x \in I_{\delta}$. 
Comparing the RHS of (4.41) to the formulas obtained after (4.14) we conclude that if $0<x<\delta^{7 / 96}$ then the RHS of (4.41) is bounded by $O\left(\delta^{1 / 32}\right)$. Combining this with the Chebyshev inequality applied to (4.37) we conclude

$$
w_{\varepsilon}(x, 0) \leq K \delta^{1 / 48}, \quad 0<x<a_{\varepsilon}-\delta^{1 / 24} .
$$

The inequalities (4.40), (4.42) now imply that the RHS of (4.31) is small for small $\delta$, uniformly in $\varepsilon, 0<\varepsilon<\delta^{1 / 4}$.

To complete the proof of the equicontinuity of the functions $\Lambda_{\varepsilon}(T)$ we need to consider the case $\delta^{1 / 4}<\varepsilon<\varepsilon_{0}$. Our goal will be to show that

$$
w_{\varepsilon}\left(x, T-\delta^{1 / 3}\right) \leq K \delta^{1 / 24}, \quad x>0,
$$

for some constant $K$ depending only on $\varepsilon_{0}, L_{0}$. It follows that $w_{\varepsilon}(x, 0)$ is also bounded by the RHS of (4.43), whence the RHS of (4.31) is small for small $\delta$, uniformly in $\varepsilon, \delta^{1 / 4}<\varepsilon<\varepsilon_{0}$. To establish (4.43) we shall use perturbation theory to solve the terminal-boundary value problem for $w_{\varepsilon}(x, t)$ on the domain $0<x<L_{0}, T-\delta^{1 / 4}<t<T$. The terminal data satisfies (4.33). The boundary data at $x=0$ is zero and we may estimate the boundary data at $x=L_{0}$ from (4.33), (4.37). Thus since $t>T-\delta^{1 / 4}$ we have as before from the Chebyshev inequality that

$$
w_{\varepsilon}\left(L_{0}, t\right) \leq K \delta^{1 / 4}, \quad T-\delta^{1 / 4}<t<T,
$$

again for a constant $K$ depending only on $\varepsilon_{0}, L_{0}$.

We proceed in a similar way to Lemma 3.3. Thus we write $w_{\varepsilon}(x, t)=w_{1, \varepsilon}(x, t)+$ $w_{2, \varepsilon}(x, t)$ where $w_{1, \varepsilon}$ has zero boundary data and $w_{2, \varepsilon}$ has zero terminal data. To estimate $w_{1, \varepsilon}$, let $G_{D}(x, y, t)$ be the Dirichlet Green's function for the interval $\left[0, L_{0}\right]$, where $G_{D}$ is given by the formula (3.16). Similarly to (3.17) we define $K_{\varepsilon, T}(x, y, t)$ by

$$
K_{\varepsilon, T}(x, y, t)=G_{D}\left(x, y, \varepsilon(1+y / \varepsilon)^{1 / 3}(T-t)\right), 0<x, y<L_{0}, t<T .
$$

Consider now the function

$$
v_{\varepsilon}(x, t)=\int_{0}^{L_{0}} K_{\varepsilon, T}(x, y, t) w_{\varepsilon}(y, T) d y, t<T .
$$

Then one has that

$$
\frac{\partial v_{\varepsilon}}{\partial t}+\varepsilon(1+x / \varepsilon)^{1 / 3} \frac{\partial^{2} v_{\varepsilon}}{\partial x^{2}}+\left[\left\{\frac{x}{L_{\varepsilon}(t)}\right\}^{1 / 3}-1\right] \frac{\partial v_{\varepsilon}}{\partial x}=g_{\varepsilon}(x, t),
$$

where $g_{\varepsilon}$ may be estimated as in Lemma 3.1. Observing that

$$
\left|\varepsilon(1+x / \varepsilon)^{1 / 3}-\varepsilon(1+y / \varepsilon)^{1 / 3}\right| \leq|x-y|,
$$

we conclude that there is a constant $C$ depending only on $\varepsilon, L_{0}$ such that

$$
\left|g_{\varepsilon}(x, t)\right| \leq C / \sqrt{\varepsilon(T-t)}, \quad t<T .
$$

We conclude that

$$
\left|w_{1, \varepsilon}(x, t)\right| \leq v_{\varepsilon}(x, t)+C \sqrt{(T-t) / \varepsilon} .
$$

To estimate $w_{2, \varepsilon}$ we note that $w_{2, \varepsilon}$ has the representation,

$$
w_{2, \varepsilon}(x, t)=E\left[w_{\varepsilon}\left(L_{0}, \tau_{x, t}\right) ; \tau_{x, t}<T, X_{\varepsilon}\left(\tau_{x, t}\right)=L_{0} \mid X_{\varepsilon}(t)=x\right],
$$


where $\tau_{x, t}$ is the first exit time from $\left[0, L_{0}\right]$ for the diffusion $X_{\varepsilon}(s), s>t$, with $X_{\varepsilon}(t)=x$. Hence from (4.44) we conclude that

$$
w_{2, \varepsilon}(x, t) \leq K \delta^{1 / 4}, T-\delta^{1 / 4}<t<T .
$$

The inequality (4.43) follows now from (4.46), (4.48) since $\varepsilon>\delta^{1 / 4}$. This completes the proof of the equicontinuity of the functions $\Lambda_{\varepsilon}(T), 0<\varepsilon<\varepsilon_{0}$, in the interval $0<T<\Lambda(0) / 4$.

Next we show equicontinuity of the functions $L_{\varepsilon}(\cdot), 0<\varepsilon<\varepsilon_{0}$, in the same interval. Analogously to (4.20) we show that for any $\eta>0$ there exists $x_{\eta}>0$ such that

$$
\int_{x_{\eta}}^{\infty} d x x^{-2 / 3} \int_{x}^{\infty} c_{\varepsilon}\left(x^{\prime}, T\right) d x^{\prime}<\eta
$$

for all $\varepsilon, 0<\varepsilon<\varepsilon_{0}$. Observe that for $A>0$,

$$
\int_{A}^{\infty} d x \int_{x}^{\infty} c_{\varepsilon}\left(x^{\prime}, T\right) d x^{\prime}=\int_{0}^{\infty} d z c_{0}(z) \int_{A}^{\infty} d x P\left(X_{\varepsilon}(T)>x ; \tau_{z, 0}>T \mid X_{\varepsilon}(0)=z\right) .
$$

From (4.35) there is the inequality,

$$
P\left(X_{\varepsilon}(T)>x ; \tau_{z, 0}>T \mid X_{\varepsilon}(0)=z\right) \leq \min \left[1, K(z+1)^{2} / x^{2}\right],
$$

for some constant $K$ depending only on $L_{0}, \varepsilon_{0}, T$. Hence

$$
\int_{A}^{\infty} d x P\left(X_{\varepsilon}(T)>x ; \tau_{z, 0}>T \mid X_{\varepsilon}(0)=z\right) \leq K_{1} \min \left[z+1,(z+1)^{2} / A\right]
$$

for a constant $K_{1}$ depending only on $L_{0}, \varepsilon_{0}, T$. It follows easily from (4.51) that the RHS of (4.50) can be made arbitrarily small by choosing $A$ sufficiently large. Now the existence of $x_{\eta}$ satisfying (4.49) follows.

To complete the proof of equicontinuity of $L_{\varepsilon}(\cdot)$ we need to show that

$$
\int_{0}^{2 x_{\eta}} d x x^{-2 / 3} \int_{x}^{\infty}\left[c_{\varepsilon}\left(x^{\prime}, T\right)-c_{\varepsilon}\left(x^{\prime}, T+\delta\right)\right] d x^{\prime}<\eta
$$

for sufficiently small $\delta>0$, provided $0<\varepsilon<\varepsilon_{0}$. As in (4.31) we write

$$
\int_{a}^{\infty}\left[c_{\varepsilon}(x, T)-c_{\varepsilon}(x, T+\delta)\right] d x=\int_{0}^{\infty} w_{\varepsilon, a}(x, 0) c_{0}(x) d x .
$$

Thus to prove (4.52) we need to get estimates on the functions $w_{\varepsilon, a}$ which are similar to the estimates we obtained for $a=0$, but which are uniform for $a$ satisfying $0 \leq a \leq 2 x_{\eta}$. This is a straightforward extension of the method we used for the case $a=0$.

We have proved equicontinuity of $\Lambda_{\varepsilon}(T), L_{\varepsilon}(T), 0<\varepsilon<\varepsilon_{0}$, in the interval $0<T<\Lambda(0) / 4$. We wish to extend this now to arbitrary values of $T$. To see this suppose $L_{\varepsilon}(\cdot)$ is bounded below by $L_{0}>0$ in the interval $[0, T]$. Then there is a constant $A$ depending only on $L_{0}, T, \varepsilon_{0}$ such that

$$
\int_{A}^{\infty} d x \int_{x}^{\infty} c_{\varepsilon}\left(x^{\prime}, T\right) d x^{\prime}<1 / 8,0<\varepsilon<\varepsilon_{0} .
$$

This follows from (4.50) and 4.51). To show that $L_{\varepsilon}(\cdot), \Lambda_{\varepsilon}(\cdot)$ remain bounded beyond the interval $[0, T]$ we note that

$$
\int_{\Lambda_{\varepsilon}(T) / 2}^{\infty} d x \int_{x}^{\infty} c_{\varepsilon}\left(x^{\prime}, T\right) d x^{\prime}+\frac{\Lambda_{\varepsilon}(T)}{2} \int_{\Lambda_{\varepsilon}(T) / 2}^{\infty} c_{\varepsilon}\left(x^{\prime}, T\right) d x^{\prime} \geq 1 / 2 .
$$


From 4.54), 4.55) we see that there is a constant $K>0$ depending only on $L_{0}, T, \varepsilon_{0}$ and $\Lambda_{\varepsilon}(T)$ such that

$$
\int_{\Lambda_{\varepsilon}(T) / 2}^{\infty} c_{\varepsilon}\left(x^{\prime}, T\right) d x^{\prime} \geq K, \quad 0<\varepsilon<\varepsilon_{0} .
$$

We proceed now as previously using the same methodology as we used to prove (4.29).

Theorem 4.1. Let $c_{\varepsilon}(x, t), L_{\varepsilon}(t), 0<x, t<\infty$, be the solution to the diffusive $L S W$ problem (1.7), (1.11) with initial data $c_{0}(x)$ satisfying

$$
\int_{0}^{\infty}(1+x) c_{0}(x) d x<\infty, \quad \int_{0}^{\infty} x c_{0}(x) d x=1 .
$$

Denote by $c_{0}(x, t), L(t)$, the solution of the $L S W$ problem (1.6), 1.7) with the same initial data. Then there are for all $x, t \geq 0$ the limits,

$$
\begin{aligned}
\lim _{\varepsilon \rightarrow 0} \int_{x}^{\infty} c_{\varepsilon}\left(x^{\prime}, t\right) d x^{\prime} & =\int_{x}^{\infty} c_{0}\left(x^{\prime}, t\right) d x^{\prime}, \\
\lim _{\varepsilon \rightarrow 0} L_{\varepsilon}(t) & =L(t) .
\end{aligned}
$$

Proof. From Lemma 4.2 we have that the functions $L_{\varepsilon}(t), 0<\varepsilon<\varepsilon_{0}$, are equicontinuous on any finite interval $[0, T]$. Hence there is a subsequence $\varepsilon_{j}, j=1,2, \ldots$, with $\lim _{j \rightarrow \infty} \varepsilon_{j}=0$ such that $L_{\varepsilon}(t)$ converges uniformly on the interval $[0, T]$ as $\varepsilon \rightarrow 0$ through the sequence $\left\{\varepsilon_{j}\right\}$ to a continuous function $L(t)$. By Lemma 4.1 it follows that the first identity in (4.56) holds, where $c_{0}(x, t)$ is the solution to (1.6). Since (1.7) also holds for $c_{\varepsilon}(\cdot, t)$, it follows from (4.56) that (1.7) must hold for $c_{0}(\cdot, t)$, whence $c_{0}(x, t)$ is the solution to the LSW problem (1.6), (1.7). By uniqueness for the solution to the LSW problem, we can then conclude that (4.56) holds as $\varepsilon \rightarrow 0$ through the reals.

\section{Convergence of Coarsening Rate}

Finally we wish to show that the rate of coarsening for the diffusive LSW problem (1.7), (1.11) converges as $\varepsilon \rightarrow 0$ to the rate of coarsening for the LSW model (1.6), (1.7). To do this we will prove that

$$
\lim _{\varepsilon \rightarrow 0} \frac{d}{d T} \int_{0}^{\infty} c_{\varepsilon}(x, T) d x=\frac{d}{d T} \int_{0}^{\infty} c_{0}(x, T) d x,
$$

where $c_{\varepsilon}$ and $c_{0}$ are as in Theorem 4.1. Evidently (1.15) follows from Theorem 4.1 and (5.1). Observe that from (4.2), (4.3) the derivative on the RHS of (5.1) exists provided the function $c_{0}(\cdot)$ is continuous at $x=F(0, T)$. We show next that the derivative on the LHS of (5.1) exists for all $\varepsilon>0$.

Lemma 5.1. Suppose the initial data $c_{0}(x)$ for the diffusive LSW problem (1.7), (1.11) satisfies the conditions of Theorem 4.1. Then for $\varepsilon, T>0$ the function $\int_{0}^{\infty} c_{\varepsilon}(x, T) d x$ is differentiable w.r. to $T$.

Proof. Let $w_{\varepsilon}(x, t, T), t<T, x>0$, be the solution of $\partial w_{\varepsilon} / \partial t=-\mathcal{L}_{t, \varepsilon}^{*} w_{\varepsilon}, w_{\varepsilon}(x, T, T)=$ $1, w_{\varepsilon}(0, t, T)=0, t<T$, where $\mathcal{L}_{t, \varepsilon}^{*}$ is given by (4.4). Then we have

$$
\int_{0}^{\infty} c_{\varepsilon}(x, T) d x=\int_{0}^{\infty} w_{\varepsilon}(x, 0, T) c_{0}(x) d x .
$$


We shall show by perturbation theory that for $t<T$ such that $T-t$ is sufficiently small, the function $w_{\varepsilon}(x, t, T)$ is differentiable w.r. to $T$ and the derivative is a bounded function. This will prove the result since the function $v_{\varepsilon}(x, t, T)=$ $-\partial w_{\varepsilon}(x, t, T) / \partial T$ satisfies $\partial v_{\varepsilon} / \partial t=-\mathcal{L}_{t, \varepsilon}^{*} v_{\varepsilon}, t<T$, with boundary condition $v_{\varepsilon}(0, t, T)=0$.

Observe that $1-w_{\varepsilon}$ satisfies the diffusion equation with zero terminal data and boundary data 1 at $x=0$. We shall show how to construct this function using perturbation theory. We first restrict ourselves to some finite interval $0<x<\varepsilon$. Let $w_{1, \varepsilon}(x, t, T), t<T, 0<x<\varepsilon$, be the solution of $\partial w_{1, \varepsilon} / \partial t=$ $-\mathcal{L}_{t, \varepsilon}^{*} w_{1, \varepsilon}, w_{1, \varepsilon}(x, T, T)=0, w_{1, \varepsilon}(0, t, T)=1, w_{1, \varepsilon}(\varepsilon, t, T)=0, t<T, 0<x<\varepsilon$. Just as in (3.22) $w_{1, \varepsilon}$ can be represented in terms of the Dirichlet Green's function $G_{\varepsilon}(x, y, t, s)$ for the interval. Thus

$$
w_{1, \varepsilon}(x, t, T)=\varepsilon \int_{t}^{T} d s \frac{\partial G_{\varepsilon}}{\partial y}(x, 0, t, s) .
$$

As in (3.23) we can represent $G_{\varepsilon}$ in a series expansion,

$$
\begin{aligned}
G_{\varepsilon}(x, y, t, T) & =K_{\varepsilon, T}(x, y, t)-\sum_{n=0}^{\infty} v_{n, \varepsilon, T}(x, y, t), \\
v_{n, \varepsilon, T}(x, y, t) & =-\int_{t}^{T} d s \int_{0}^{\varepsilon} d y^{\prime} K_{\varepsilon, s}\left(x, y^{\prime}, t\right) g_{n, \varepsilon, T}\left(y^{\prime}, y, s\right), \\
g_{0, \varepsilon, T}(x, y, t) & =g_{\varepsilon, T}(x, y, t)=\left[\frac{\partial}{\partial t}+\mathcal{L}_{t, \varepsilon}^{*}\right] K_{\varepsilon, T}(x, y, t), \\
g_{n+1, \varepsilon, T} & =g_{n, \varepsilon, T}-\left\{\frac{\partial}{\partial t}+\mathcal{L}_{t, \varepsilon}^{*}\right\} v_{n, \varepsilon, T}, n \geq 0,
\end{aligned}
$$

where $K_{\varepsilon, T}$ is given by (4.45). The function $g_{n, \varepsilon, T}$ is given by the recursion formula,

$$
g_{n+1, \varepsilon, T}(x, y, t)=\int_{t}^{T} d s \int_{0}^{\varepsilon} d y^{\prime}\left\{\frac{\partial}{\partial t}+\mathcal{L}_{t, \varepsilon}^{*}\right\} K_{\varepsilon, s}\left(x, y^{\prime}, t\right) g_{n, \varepsilon, T}\left(y^{\prime} y, s\right) .
$$

Now as in (3.24) there is a universal constant $C>0$ such that

$$
\left|g_{n, \varepsilon, T}(x, y, t)\right| \leq \frac{C^{n}(T-t)^{n / 2-1 / 2}}{\varepsilon^{(n+1) / 2}} G(x-y, 2 \varepsilon(T-t)), n \geq 0 .
$$

Hence the series (5.3) converges for $T-t<\varepsilon / C$. If we formally differentiate the RHS of (5.5) w.r. to $T$ we are led to expect the inequality,

$$
\left|\partial g_{n, \varepsilon, T}(x, y, t) / \partial y\right| \leq \frac{C^{n}(T-t)^{n / 2-1}}{\varepsilon^{(n / 2+1)}} G(x-y, 2 \varepsilon(T-t)), n \geq 0 .
$$

It is easy to see that (5.6) holds for $n=0$. For $n=1$ we use (5.4). Writing

$$
g_{1, \varepsilon, T}=\int_{t}^{(T+t) / 2} d s+\int_{(T+t) / 2}^{T} d s
$$

one can easily see that the derivative of the first integral on the RHS of (5.7) w.r. to $y$ is bounded by the RHS of (5.6) for $n=1$. To estimate the derivative of the second integral we need to integrate by parts. We argue as in (3.27). Thus

$$
\left\{\frac{\partial}{\partial s}+\mathcal{L}_{\varepsilon, y^{\prime}}^{*}\right\} \frac{\partial K_{\varepsilon, T}}{\partial y}\left(y^{\prime}, y, s\right)=\left[\left\{\frac{y^{\prime}}{L_{\varepsilon}(s)}\right\}^{1 / 3}-1\right] \frac{\partial^{2} K_{\varepsilon, T}}{\partial y^{\prime} \partial y}
$$




$$
+\frac{\left(1+y^{\prime} / \varepsilon\right)^{1 / 3}}{3 \varepsilon(1+y / \varepsilon)^{4 / 3}} \frac{\partial K_{\varepsilon, T}}{\partial s}+\left[1-\frac{\left(1+y^{\prime} / \varepsilon\right)^{1 / 3}}{(1+y / \varepsilon)^{1 / 3}}\right] \frac{\partial^{2} K_{\varepsilon, T}}{\partial s \partial y} .
$$

We substitute the RHS of (5.8) into the second integral in (5.7) as the expression for $\partial g_{0, \varepsilon, T}\left(y^{\prime}, y, s\right) / \partial y$. We then integrate by parts w.r. to $y^{\prime}$ for the first term, and $s$ for the last two terms in (5.8). It is easy to see from this that (5.6) holds for $n=1$. Hence (5.6) holds by induction for all $n \geq 0$. It follows then from (5.3) that

$$
\left|\partial v_{n, \varepsilon, T}(x, y, t) / \partial y\right| \leq \frac{C^{n}(T-t)^{n / 2}}{\varepsilon^{n / 2+1}} G(x-y, 2 \varepsilon(T-t)), n \geq 0 .
$$

Note that to obtain (5.9) for $n=0$ we need to use the representation (5.8) and integrate by parts. Hence if $(T-t)<\varepsilon / C$ the series in (5.3) for the derivative $\partial G_{\varepsilon}(x, y, t, T) / \partial y$ converges, and we conclude that

$$
\left|\partial G_{\varepsilon}(x, y, t, T) / \partial y\right| \leq \frac{C}{\sqrt{\varepsilon(T-t)}} G(x-y, 2 \varepsilon(T-t)),
$$

for some constant $C$. Now from (5.2), (5.10) we see that $w_{1, \varepsilon}(x, t, T)$ is differentiable w.r. to $T$ and is given by the formula

$$
\frac{\partial w_{1, \varepsilon}}{\partial T}(x, t, T)=\varepsilon \frac{\partial G_{\varepsilon}}{\partial y}(x, 0, t, T) .
$$

We return to consideration of the function $w_{\varepsilon}(x, t, T)$. If $X_{\varepsilon}(s)$ is the diffusion process associated with $\mathcal{L}_{s, \varepsilon}^{*}$ then

$$
1-w_{\varepsilon}(x, t, T)=P\left(\tau_{x, t}<T \mid X_{\varepsilon}(t)=x\right),
$$

where $\tau_{x, t}$ is the first hitting time at 0 for $X_{\varepsilon}(s), s \geq t$. Suppose that $0<x<\varepsilon$ and let $\tau_{1, x, t}$ be the first exit time from the interval $[0, \varepsilon]$ for $X_{\varepsilon}(s), s \geq t$, with $X_{\varepsilon}(t)=x$. If $X_{\varepsilon}\left(\tau_{1, x, t}\right)=\varepsilon$ denote by $\tau_{2, x, t}>\tau_{1, x, t}$ the first hitting time at $\varepsilon / 2$. The density $d \mu_{x, t}(s), s>t$, associated with $\tau_{2, x, t}$ is defined by

$$
P\left(\tau_{2, x, t}<T ; X_{\varepsilon}\left(\tau_{1, x, t}\right)=\varepsilon\right)=\int_{t}^{T} d \mu_{x, t}(s) .
$$

We can use this density to write $1-w_{\varepsilon}$ in terms of the function $w_{1, \varepsilon}$. Thus

$$
\begin{aligned}
1-w_{\varepsilon}(x, t, T) & =w_{1, \varepsilon}(x, t, T)+\int_{t}^{T} d \mu_{x, t}(s) w_{1, \varepsilon}(\varepsilon / 2, s, T) \\
& +\int_{t<s_{1}<s_{2}<T} d \mu_{x, t}\left(s_{1}\right) d \mu_{\varepsilon / 2, s_{1}}\left(s_{2}\right) w_{1, \varepsilon}\left(\varepsilon / 2, s_{2}, T\right)+\cdots
\end{aligned}
$$

It is evident that the series converges and is term by term differentiable w.r. to $T$, provided $(T-t)<<\varepsilon$.

Let us write $\rho_{\varepsilon}(x, t, T)=-\partial w_{\varepsilon}(x, t, T) / \partial T$ where the function $w_{\varepsilon}(x, t, T)$ is given in Lemma 5.1. Then $\rho_{\varepsilon}(x, t, \cdot)$ is the density for the exit time $\tau_{x, t}$ at 0 of the diffusion $X_{\varepsilon}(s)$ given by (4.7) with $X_{\varepsilon}(t)=x$.

Lemma 5.2. There are positive constants $c_{1}, C_{1}>0$ such that if $T-t=c_{1} \varepsilon$ then $\rho_{\varepsilon}$ satisfies the inequality,

$$
\rho_{\varepsilon}(x, t, T) \leq \frac{C_{1}}{\varepsilon} \exp \left[-(x / 4 \varepsilon)^{5 / 3}\right], \quad x \geq 0 .
$$


Proof. The result follows from Lemma 5.1 provided $0<x<\varepsilon$. For $x>\varepsilon$ let $\tau_{x, t}$ be the first hitting time at $\varepsilon / 2$ for the diffusion $X_{\varepsilon}(s)$ with $X_{\varepsilon}(t)=x$. Then from the formula (5.12) it will be sufficient to show that

$$
P\left(\tau_{x, t}<T\right) \leq C_{1} \exp \left[-(x / 4 \varepsilon)^{5 / 3}\right] .
$$

For any non-negative integer $N$ let $I_{N}$ be the interval $\left[2^{N} \varepsilon, 2^{N+1} \varepsilon\right]$, and $Z_{\varepsilon}(s)$ the diffusion process started at $Z_{\varepsilon}(t)=x \in I_{N}$ which satisfies the stochastic equation,

$$
d Z_{\varepsilon}(s)=-d s+\sqrt{2 \varepsilon}\left(1+Z_{\varepsilon}(s) / \varepsilon\right)^{1 / 6} d W(s) .
$$

We denote by $\tau_{1, x, t}$ the exit time from $I_{N}$ for the process $Z_{\varepsilon}(s)$ with $Z_{\varepsilon}(t)=x$. Evidently one has $P\left(\tau_{x, t}<T\right) \leq P\left(\tau_{1, x, t}<T\right)$. We can generate the density for $\tau_{1, x, t}$ by perturbation theory as in Lemma 5.1. The series converges provided $(T-t) / 2^{N / 3} \varepsilon<<1$ If we take $x \sim 2^{N+1 / 2} \varepsilon$ then one sees that provided $T-t=c_{1} \varepsilon$ for some sufficiently small universal constant $c_{1}$ there is the inequality,

$$
P\left(\tau_{1, x, t}<t+c_{1} \varepsilon\right)<C_{1} \exp \left[-\left(2^{N} / 10\right)^{5 / 3}\right],
$$

where $C_{1}$ is also a universal constant. The inequality (5.13) follows.

From Lemma 5.2 we see that the integral of the function $\rho_{\varepsilon}(\cdot, t, T)$ is bounded independent of $\varepsilon$ as $\varepsilon \rightarrow 0$ provided $T-t \sim \varepsilon$. We shall show that the integral is in fact close to 1 for $T-t=O(1)$ as $\varepsilon \rightarrow 0$.

Lemma 5.3. There exists $\delta_{0}>0$ such that if $\delta$ satisfies $0<\delta<\delta_{0}$ then one can find $\varepsilon(\delta)>0$ for which the following inequality holds:

$$
\left|1-\int_{0}^{\infty} \rho_{\varepsilon}(x, t, T) d x\right|<\delta^{1 / 10}, T-t=\delta, 0<\varepsilon<\varepsilon(\delta) .
$$

Proof. We first show that (5.15) holds in an averaged sense. Thus let $0<\eta<\delta / 2$. We shall see that there exists $\varepsilon(\delta, \eta)>0$ such that for $0<\varepsilon<\varepsilon(\delta, \eta)$ there is the inequality,

$$
\left|1-\frac{1}{2 \eta} \int_{t+\delta-\eta}^{t+\delta+\eta} d T \int_{0}^{\infty} \rho(x, t, T) d x\right|<\delta^{1 / 4} .
$$

Let $\tau_{x, t}$ be the first hitting time at 0 for the diffusion process $X_{\varepsilon}(s)$ associated with $\mathcal{L}_{s, \varepsilon}^{*}$, where $X_{\varepsilon}(t)=x$. Then (5.16) is the same as

$$
\left|1-\frac{1}{2 \eta} \int_{0}^{\infty} P\left(t+\delta-\eta<\tau_{x, t}<t+\delta+\eta\right) d x\right|<\delta^{1 / 4} .
$$

Now (5.17) will follow if we can estimate $P\left(\tau_{x, t}>T\right)$ sufficiently accurately for $T-t \sim \delta$. In particular we show that $P\left(\tau_{x, t}>T\right) \sim 1$ if $x$ is slightly larger than $T-t$ (depending on $\delta$ and $\varepsilon$ ) and $P\left(\tau_{x, t}>T\right) \sim 0$ if $x$ is slightly less than $T-t$. To do this we proceed as in Lemma 4.1. We consider the situation when $x$ is large. For $Z_{\varepsilon}(s)$ satisfying (5.14) let $\tau_{x}$ be the first exit time for the diffusion $Z_{\varepsilon}(s)$ with $Z_{\varepsilon}(t)=x$ from the interval $[x / 2,2 x]$. Then $P\left(\tau_{x, t}<T\right) \leq P\left(\tau_{x}<T\right)$. Now one has

$$
Z_{\varepsilon}\left(s \wedge \tau_{x}\right)=x-\left[s \wedge \tau_{x}-t\right]+\int_{t}^{s \wedge \tau_{x}} \sqrt{2 \varepsilon}\left[1+Z_{\varepsilon}\left(s^{\prime}\right) / \varepsilon\right]^{1 / 6} d W\left(s^{\prime}\right)
$$


It follows that provided $(T-t)<x / 4$ there is the inequality,

$$
P\left(\tau_{x}<T\right) \leq\left(\frac{4}{x}\right)^{2} 2 \varepsilon[1+2 x / \varepsilon]^{1 / 3}(T-t) .
$$

Hence one can choose $\varepsilon(\delta)$ so that if $\varepsilon<\varepsilon(\delta)$ the integral in (5.17) over $x>8 \delta$ is negligible. For $x<8 \delta$ we see just as in the derivation of (4.14) that

$$
\lim _{\varepsilon \rightarrow 0} P\left(\tau_{x, t}>t+\eta^{\prime}+x\left[1+K \delta^{1 / 3}\right]\right)=0, \quad \lim _{\varepsilon \rightarrow 0} P\left(\tau_{x, t}<t-\eta^{\prime}+x\right)=0,
$$

for any $\eta^{\prime}>0$, where $K$ is a constant independent of $\varepsilon$ and $\delta$. The inequality (5.17) follows from (5.18) and (5.19).

To obtain the pointwise estimate (5.15) we use the fact that $\rho_{\varepsilon}(x, t, T)$ satisfies the equation $\partial \rho_{\varepsilon} / \partial t+\mathcal{L}_{t, \varepsilon}^{*} \rho_{\varepsilon}=0, \rho_{\varepsilon}(0, t, T)=0$. Let $c_{\varepsilon}(x, s), s>t$, satisfy the adjoint equation $\partial c_{\varepsilon} / \partial s=\mathcal{L}_{s, \varepsilon} c_{\varepsilon}, c_{\varepsilon}(0, s)=0, s>t, c_{\varepsilon}(x, t)=1, x>0$. Then one has that

$$
\int_{0}^{\infty} \rho_{\varepsilon}(x, t, T) d x=\int_{0}^{\infty} \rho_{\varepsilon}(x, s, T) c_{\varepsilon}(x, s) d x, \quad s>t .
$$

From (1.11) we see that $c_{\varepsilon}(x, t)$ is given by the expectation value,

$c_{\varepsilon}(x, s)=E\left[\exp \left\{-\int_{t}^{s} \frac{2}{9 \varepsilon}\left(1+X_{\varepsilon}\left(s^{\prime}\right) / \varepsilon\right)^{-5 / 3}+\frac{1}{3\left(X_{\varepsilon}\left(s^{\prime}\right)^{2} L_{\varepsilon}\left(s^{\prime}\right)\right)^{1 / 3}} d s^{\prime}\right\} ; \tau_{x, s}<t\right]$,

where $X_{\varepsilon}\left(s^{\prime}\right)$ is the diffusion process satisfying

$$
\begin{aligned}
d X_{\varepsilon}\left(s^{\prime}\right)=-\left\{1-\left[\frac{X_{\varepsilon}\left(s^{\prime}\right)}{L_{\varepsilon}\left(s^{\prime}\right)}\right]^{1 / 3}\right. & \left.+\frac{2}{3}\left(1+\frac{X_{\varepsilon}\left(s^{\prime}\right)}{\varepsilon}\right)^{-2 / 3}\right\} d s^{\prime} \\
& +\sqrt{2 \varepsilon}\left(1+X_{\varepsilon}\left(s^{\prime}\right) / \varepsilon\right)^{1 / 6} d W\left(s^{\prime}\right), s^{\prime}<s,
\end{aligned}
$$

with $X_{\varepsilon}(s)=x$. Note that the process $X_{\varepsilon}\left(s^{\prime}\right)$ is running backwards in time. The stopping time $\tau_{x, s}$ is the first hitting time for the process on the boundary $x=0$. From (5.21) we see that $c_{\varepsilon}(x, s)<1, s>t$. Hence by taking $T-s \sim \varepsilon$ we can conclude from Lemma 5.2 that the LHS of (5.20) is uniformly bounded as $\varepsilon \rightarrow 0$ for any fixed $t<T$. Let $\rho_{\varepsilon}^{\prime}(x, t, T)$ be the density for the diffusion $Z_{\varepsilon}$ which corresponds to the density $\rho_{\varepsilon}(x, t, T)$ for $X_{\varepsilon}$. Using the time translation invariance of $Z_{\varepsilon}$ we have from the inequality (5.16) for $\rho_{\varepsilon}^{\prime}$ that

$$
\left|1-\frac{1}{2 \eta} \int_{T-\delta}^{T-\delta+2 \eta} d s \int_{0}^{\infty} \rho_{\varepsilon}^{\prime}(x, s, T) d x\right|<\delta^{1 / 4},
$$

for $0<\varepsilon<\varepsilon(\delta, \eta)$. Now from equation (5.20) for $\rho_{\varepsilon}^{\prime}$ one has that

$$
\int_{0}^{\infty} \rho_{\varepsilon}^{\prime}(x, T-\delta, T) d x=\frac{1}{2 \eta} \int_{T-\delta}^{T-\delta+2 \eta} d s \int_{0}^{\infty} \rho_{\varepsilon}^{\prime}(x, s, T) c_{\varepsilon}^{\prime}(x, s) d x,
$$

where $c_{\varepsilon}^{\prime}$ is given by an expectation similar to (5.21). Using the fact that the integral on the RHS of (5.24) is concentrated at $x \sim \delta$ and (5.21) for $c_{\varepsilon}^{\prime}$, we conclude from (5.23) that one can choose $\varepsilon(\delta)$ such that for $0<\varepsilon<\varepsilon(\delta)$ one has the pointwise in time estimate

$$
\left|1-\int_{0}^{\infty} \rho_{\varepsilon}^{\prime}(x, T-\delta, T) d x\right|<\delta^{1 / 4} .
$$


The inequality (5.15) follows if we can show that $\rho_{\varepsilon}^{\prime}(\cdot, T-\delta, T)$ and $\rho_{\varepsilon}(\cdot, T-\delta, T)$ are close.

We first compare $\rho_{\varepsilon}(x, t, T)$ and $\rho_{\varepsilon}^{\prime}(x, t, T)$ when $T-t=c_{1} \varepsilon$ as in Lemma 5.2. To do this we write $\rho_{\varepsilon}(x, t, T)$ as a sum over walks on the numbers $0,1 / 2,1,2, \ldots, 2^{N}$, where $2^{N} \varepsilon \sim 1$. For a walk $X(n), n=0,1,2, \ldots$ we take $X(0)>0$. If $0<X(n)<$ $2^{N}$ then $X(n+1)$ can be either of the neighbors of $X(n)$. If $X(n)=2^{N}$ then $X(n+1)=0$. Finally let $\tau$ be defined by $X(\tau)=0$ and $X(n)>0, n<\tau$. Then for $x=2^{n} \varepsilon$ for some integer $n$ with $-1 \leq n \leq N$ we have that

$$
\begin{aligned}
\rho_{\varepsilon}(x, t, T)= & \sum_{\{\text {walks } X(\cdot): X(0)=x / \varepsilon\}} \int_{s_{0}<s_{1}<s_{2} . .<s_{\tau}} \delta\left(s_{0}-t\right) \delta\left(s_{\tau}-T\right) \\
d s_{0} d s_{1} \ldots d s_{\tau} & \prod_{n=1}^{\tau} \rho_{\varepsilon}\left(\varepsilon X(n-1), \varepsilon X(n), s_{n-1}, s_{n}\right) .
\end{aligned}
$$

Here $\rho_{\varepsilon}\left(x, x^{\prime}, s, s^{\prime}\right)$ is the density at $x^{\prime}$ at time $s^{\prime}$ for the diffusion $X_{\varepsilon}(\cdot)$ of (4.7) with $X_{\varepsilon}(s)=x$ exiting the interval $I_{x}$ where $I_{x}=[x / 2,2 x]$ if $x=2^{n} \varepsilon$ with $0 \leq n<$ $N, I_{x}=[0, \varepsilon]$ if $x=\varepsilon / 2$. For $x=2^{N} \varepsilon$ then $x^{\prime}=0$ and $\rho_{\varepsilon}\left(x, x^{\prime}, s, s^{\prime}\right)=\rho_{\varepsilon}\left(x, s, s^{\prime}\right)$. Just as we derived (5.10) we see that there are positive universal constants $C_{1}, C_{2}$ such that

$$
\rho_{\varepsilon}\left(x, x^{\prime}, s, s^{\prime}\right) \leq \frac{C_{1}}{s^{\prime}-s} \exp \left[-C_{2} x^{5 / 3} / \varepsilon^{2 / 3}\left(s^{\prime}-s\right)\right] .
$$

It follows that we may assign transition probabilities on the walks $X(\cdot)$ so that

$$
\rho_{\varepsilon}(x, t, T) \leq \frac{C}{\varepsilon} E\left\{\exp \left[-\eta \sum_{n=0}^{\tau} X(n)^{5 / 3}\right] \mid X(0)=x / \varepsilon\right\},
$$

for some positive constants $C, \eta$. This last inequality gives another proof of Lemma 5.2. To compare $\rho_{\varepsilon}$ and $\rho_{\varepsilon}^{\prime}$ we use the representation (5.25) and an interpolation formula. Thus we have

$$
\begin{gathered}
\rho_{\varepsilon}(x, t, T)-\rho_{\varepsilon}^{\prime}(x, t, T)=\int_{0}^{1} d \lambda \sum_{\{\text {walks } X(\cdot): X(0)=x / \varepsilon\}} \int_{s_{0}<s_{1}<s_{2} . .<s_{\tau}} \delta\left(s_{0}-t\right) \delta\left(s_{\tau}-T\right) d s_{0} d s_{1} \ldots d s_{\tau} \\
\prod_{n=1}^{\tau}\left[\lambda \rho_{\varepsilon}\left(\varepsilon X(n-1), \varepsilon X(n), s_{n-1}, s_{n}\right)+(1-\lambda) \rho_{\varepsilon}^{\prime}\left(\varepsilon X(n-1), \varepsilon X(n), s_{n-1}, s_{n}\right)\right] \\
\sum_{n=1}^{\tau}\left[\rho_{\varepsilon}\left(\varepsilon X(n-1), \varepsilon X(n), s_{n-1}, s_{n}\right)-\rho_{\varepsilon}^{\prime}\left(\varepsilon X(n-1), \varepsilon X(n), s_{n-1}, s_{n}\right)\right] \\
/\left[\lambda \rho_{\varepsilon}\left(\varepsilon X(n-1), \varepsilon X(n), s_{n-1}, s_{n}\right)+(1-\lambda) \rho_{\varepsilon}^{\prime}\left(\varepsilon X(n-1), \varepsilon X(n), s_{n-1}, s_{n}\right)\right] .
\end{gathered}
$$

Now using the perturbation method of Lemma 5.1 we see that

$$
\left|\rho_{\varepsilon}\left(x, x^{\prime}, s, s^{\prime}\right)-\rho_{\varepsilon}^{\prime}\left(x, x^{\prime}, s, s^{\prime}\right)\right| \leq \frac{C_{1} x^{1 / 3}}{s^{\prime}-s} \exp \left[-C_{2} x^{5 / 3} / \varepsilon^{2 / 3}\left(s^{\prime}-s\right)\right],
$$

provided $x=2^{n} \varepsilon$ for any integer $n,-1 \leq n<N$. Since $2^{N} \varepsilon \sim 1$ the inequality (5.29) also holds for $x=2^{N} \varepsilon$. This follows from (5.26). Just as we derived (5.27) 
we have from (5.29) that

$\left|\rho_{\varepsilon}(x, t, T)-\rho_{\varepsilon}^{\prime}(x, t, T)\right| \leq \frac{C}{\varepsilon^{2 / 3}} E\left\{\sum_{n=1}^{\tau} X(n)^{1 / 3} \exp \left[-\eta \sum_{n=1}^{\tau} X(n)^{5 / 3}\right] \mid X(0)=x / \varepsilon\right\}$,

whence it follows that

$$
\left|\rho_{\varepsilon}(x, t, T)-\rho_{\varepsilon}^{\prime}(x, t, T)\right| \leq \frac{C_{1}}{\varepsilon^{2 / 3}} \exp \left[-C_{2}(x / \varepsilon)^{5 / 3}\right]
$$

provided $T-t=c_{1} \varepsilon$ and $x<O(1)$. For $x>O(1)$ the inequality (5.30) follows from Lemma 5.2.

We shall now use (5.30) and (5.20) to compare the integrals of $\rho_{\varepsilon}(\cdot, t, T)$ and $\rho_{\varepsilon}^{\prime}(\cdot, t, T)$ when $T-t=\delta$. In fact we have

$$
\begin{aligned}
& \int_{0}^{\infty} \rho_{\varepsilon}(x, t, T)-\rho_{\varepsilon}^{\prime}(x, t, T) d x= \\
& \int_{0}^{\infty}\left[\rho_{\varepsilon}\left(x, T-c_{1} \varepsilon, T\right)-\rho_{\varepsilon}^{\prime}\left(x, T-c_{1} \varepsilon, T\right)\right] c_{\varepsilon}\left(x, T-c_{1} \varepsilon\right) d x \\
& +\int_{0}^{\infty} \rho_{\varepsilon}^{\prime}\left(x, T-c_{1} \varepsilon, T\right)\left[c_{\varepsilon}\left(x, T-c_{1} \varepsilon\right)-c_{\varepsilon}^{\prime}\left(x, T-c_{1} \varepsilon\right)\right] d x,
\end{aligned}
$$

where $c_{\varepsilon}^{\prime}$ is the analogous expectation for the diffusion $Z_{\varepsilon}$ which corresponds to $c_{\varepsilon}$ for $X_{\varepsilon}$. Since $c_{\varepsilon}\left(x, T-c_{1} \varepsilon\right)<1$ it follows from (5.30) that the first integral on the RHS of (5.31) is small. We shall show the second integral is also small by obtaining pointwise estimates on the differences $c_{\varepsilon}^{\prime}(x, s)-c_{\varepsilon}(x, s)$ for $s \sim \delta+t$ and $x>0$. In fact, in view of Lemma 5.2, we may restrict ourselves to obtaining an estimate on the difference when $x=O(\varepsilon)$.

To carry this out we consider the representation for $c_{\varepsilon}^{\prime}$ analogous to the representation (5.21) for $c_{\varepsilon}$. Let $Y_{\varepsilon}\left(s^{\prime}\right)$ be the diffusion process satisfying

$d Y_{\varepsilon}\left(s^{\prime}\right)=-\left\{1+\frac{2}{3}\left(1+\frac{Y_{\varepsilon}\left(s^{\prime}\right)}{\varepsilon}\right)^{-2 / 3}\right\} d s^{\prime}+\sqrt{2 \varepsilon}\left(1+Y_{\varepsilon}\left(s^{\prime}\right) / \varepsilon\right)^{1 / 6} d W\left(s^{\prime}\right), s^{\prime}<s$,

with $Y_{\varepsilon}(s)=x$. If we allow the diffusions $X_{\varepsilon}\left(s^{\prime}\right)$ of (5.22) and $Y_{\varepsilon}\left(s^{\prime}\right)$ of (5.32) to be driven backwards in time by the same Brownian motion $d W\left(s^{\prime}\right)$ then it is clear that if $X_{\varepsilon}(s)=Y_{\varepsilon}(s)=x$ then $X_{\varepsilon}\left(s^{\prime}\right) \leq Y_{\varepsilon}\left(s^{\prime}\right), s^{\prime}<s$. Thus if $\tau_{x, s}$ is the first hitting time at 0 for $X_{\varepsilon}\left(s^{\prime}\right)$ with $X_{\varepsilon}(s)=x$ and $\tau_{x, s}^{\prime}$ is similarly defined for $Y_{\varepsilon}\left(s^{\prime}\right)$, then $\tau_{x, s} \geq \tau_{x, s}^{\prime}$. Now similarly to (5.21) we have the representation,

$$
c_{\varepsilon}^{\prime}(x, s)=E\left[\exp \left\{-\int_{t}^{s} \frac{2}{9 \varepsilon}\left(1+Y_{\varepsilon}\left(s^{\prime}\right) / \varepsilon\right)^{-5 / 3} d s^{\prime}\right\} ; \tau_{x, s}^{\prime}<t\right] .
$$

If we compare (5.21), (5.33) and use the fact that $\tau_{x, s} \geq \tau_{x, s}^{\prime}, X_{\varepsilon}\left(s^{\prime}\right) \leq Y_{\varepsilon}\left(s^{\prime}\right), s^{\prime} \leq s$, we see that $c_{\varepsilon}(x, s) \leq c_{\varepsilon}^{\prime}(x, s)$. We also have that

$$
\begin{aligned}
c_{\varepsilon}^{\prime}(x, s)-c_{\varepsilon}(x, s) & =E\left[\exp \left\{-\int_{t}^{s} \frac{2}{9 \varepsilon}\left(1+Y_{\varepsilon}\left(s^{\prime}\right) / \varepsilon\right)^{-5 / 3} d s^{\prime}\right\} ; \tau_{x, s}^{\prime}<t, \tau_{x, s}>t\right] \\
& +E\left[\exp \left\{-\int_{t}^{s} \frac{2}{9 \varepsilon}\left(1+Y_{\varepsilon}\left(s^{\prime}\right) / \varepsilon\right)^{-5 / 3} d s^{\prime}\right\}\right. \\
& \left.-\exp \left\{-\int_{t}^{s} \frac{2}{9 \varepsilon}\left(1+X_{\varepsilon}\left(s^{\prime}\right) / \varepsilon\right)^{-5 / 3}+\frac{1}{3\left[X_{\varepsilon}\left(s^{\prime}\right)^{2} L_{\varepsilon}\left(s^{\prime}\right)\right]^{1 / 3}} d s^{\prime}\right\} ; \tau_{x, s}<t\right] .
\end{aligned}
$$

We conclude from the previous inequality that 


$$
\begin{gathered}
0 \leq c_{\varepsilon}^{\prime}(x, s)-c_{\varepsilon}(x, s) \leq P\left(\tau_{x, s}^{\prime}<t, \tau_{x, s}>t\right)+E\left[\int_{t}^{s} \frac{d s^{\prime}}{3\left[X_{\varepsilon}\left(s^{\prime}\right)^{2} L_{\varepsilon}\left(s^{\prime}\right)\right]^{1 / 3}} ; \tau_{x, s}<t\right] \\
+E\left[\frac{2}{9 \varepsilon} \int_{t}^{s}\left[1+X_{\varepsilon}\left(s^{\prime}\right) / \varepsilon\right]^{-5 / 3}-\left[1+Y_{\varepsilon}\left(s^{\prime}\right) / \varepsilon\right]^{-5 / 3} d s^{\prime} ; \tau_{x, s}<t\right] .
\end{gathered}
$$

We shall estimate each term on the RHS of (5.34). Note that for the purposes of doing this estimate we may replace $L_{\varepsilon}\left(s^{\prime}\right), t \leq s^{\prime} \leq t+\delta$, on the RHS of (15.22) by $L_{0}=\inf _{t \leq s^{\prime} \leq t+\delta} L_{\varepsilon}\left(s^{\prime}\right)$.

We consider the first term which is the same as $P\left(\tau_{x, s}^{\prime}<t\right)-P\left(\tau_{x, s}<t\right)$. We shall estimate this by obtaining an upper bound on $P\left(\tau_{x, s}^{\prime}<t\right)$ and a lower bound on $P\left(\tau_{x, s}<t\right)$. We have now that for $0<x<\delta^{2}$,

(5.35) $P\left(\tau_{x, s}^{\prime}<t\right) \leq P\left(Y_{\varepsilon}\left(s^{\prime}\right)\right.$ exits $\left[0, \delta^{2}\right]$ through $\left.\delta^{2} \mid Y_{\varepsilon}(s)=x\right)$

$$
\left.+P \text { (Time to exit }\left[0, \delta^{2}\right] \text { larger than } \delta / 2 \mid Y_{\varepsilon}(s)=x\right) \text {. }
$$

Letting $u_{\varepsilon}(x)$ be the first probability on the RHS of (5.35) we have that

$$
\begin{aligned}
& \varepsilon(1+x / \varepsilon)^{1 / 3} u_{\varepsilon}^{\prime \prime}(x)+\left[1+\frac{2}{3}(1+x / \varepsilon)^{-2 / 3}\right] u_{\varepsilon}^{\prime}(x)=0, \\
& 0<x<\delta^{2}, u_{\varepsilon}(0)=0, u_{\varepsilon}\left(\delta^{2}\right)=1 .
\end{aligned}
$$

The solution to (5.36) is given by the formula,

$$
\begin{aligned}
& u_{\varepsilon}(x)=\int_{0}^{x} h_{\varepsilon}\left(x^{\prime}\right) d x^{\prime} / \int_{0}^{\delta^{2}} h_{\varepsilon}\left(x^{\prime}\right) d x^{\prime}, \\
& h_{\varepsilon}(x)=(1+x / \varepsilon)^{-2 / 3} \exp \left[-\frac{3}{2}(1+x / \varepsilon)^{2 / 3}\right] .
\end{aligned}
$$

Observe that $u_{\varepsilon}(x) \simeq 1$ for $x>O(\varepsilon)$. We estimate the second probability on the RHS of (5.35) by calculating the expected time to exit $\left[0, \delta^{2}\right]$. Letting $\tau_{x}$ be the time to exit and $v_{\varepsilon}(x)=E\left[\tau_{x}\right]$ then $v_{\varepsilon}(x)$ satisfies

$$
\begin{aligned}
-\varepsilon(1+x / \varepsilon)^{1 / 3} v_{\varepsilon}^{\prime \prime}(x)-\left[1+\frac{2}{3}(1+x / \varepsilon)^{-2 / 3}\right] & v_{\varepsilon}^{\prime}(x)=1, \\
0<x<\delta^{2}, & v_{\varepsilon}(0)=v_{\varepsilon}\left(\delta^{2}\right)=0 .
\end{aligned}
$$

We can write $v_{\varepsilon}(x)$ as an integral w.r. to the Dirichlet Green's function $G_{\varepsilon}(x, y)$ for the equation (5.38). Thus we have

$$
v_{\varepsilon}(x)=\int_{0}^{\delta^{2}} G_{\varepsilon}(x, y) d y
$$

where $G_{\varepsilon}(x, y)$ is given by the formula,

$$
G_{\varepsilon}(x, y)=\left[\int_{0}^{x} h_{\varepsilon}\left(x^{\prime}\right) d x^{\prime}\right]\left[\int_{y}^{\delta^{2}} h_{\varepsilon}\left(x^{\prime}\right) d x^{\prime}\right] / \varepsilon(1+y / \varepsilon)^{1 / 3} h_{\varepsilon}(y) \int_{0}^{\delta^{2}} h_{\varepsilon}\left(x^{\prime}\right) d x^{\prime},
$$

if $x<y$, and

$$
G_{\varepsilon}(x, y)=\left[\int_{x}^{\delta^{2}} h_{\varepsilon}\left(x^{\prime}\right) d x^{\prime}\right]\left[\int_{0}^{y} h_{\varepsilon}\left(x^{\prime}\right) d x^{\prime}\right] / \varepsilon(1+y / \varepsilon)^{1 / 3} h_{\varepsilon}(y) \int_{0}^{\delta^{2}} h_{\varepsilon}\left(x^{\prime}\right) d x^{\prime},
$$


if $x>y$. In (5.39) and (5.40) the function $h_{\varepsilon}(x)$ is given by (5.38). Observe that upon integration by parts,

$\int_{y}^{\delta^{2}} h_{\varepsilon}\left(x^{\prime}\right) d x^{\prime}=\varepsilon(1+y / \varepsilon)^{1 / 3} h_{\varepsilon}(y)-\varepsilon\left(1+\delta^{2} / \varepsilon\right)^{1 / 3} h_{\varepsilon}\left(\delta^{2}\right)-\frac{1}{3} \int_{y}^{\delta^{2}}\left(1+x^{\prime} / \varepsilon\right)^{-2 / 3} h_{\varepsilon}\left(x^{\prime}\right) d x^{\prime}$.

Hence we see from (5.39) that $G_{\varepsilon}(x, y)<1$ if $x<y$. Using the fact that $(1+$ $x / \varepsilon)^{1 / 3} h_{\varepsilon}(x)$ is a decreasing function, we similarly see from (5.40) that $G_{\varepsilon}(x, y)<1$ for $x>y$. Thus $v_{\varepsilon}(x)<\delta^{2}$, whence by Chebyshev the second term on the RHS of (5.35) is smaller than $2 \delta$.

To obtain a lower bound on $P\left(\tau_{x, s}<t\right)$ observe first that

$$
\begin{aligned}
P\left(\tau_{x, s}<t\right) \geq & P\left(X_{\varepsilon}\left(s^{\prime}\right) \text { exits }[0, \sqrt{\delta}] \text { through } \sqrt{\delta} \mid X_{\varepsilon}(s)=x\right) \\
& \times P\left(\text { Time to exit }[0,2 \sqrt{\delta}] \text { is larger than } \delta \mid X_{\varepsilon}(s)=\sqrt{\delta}\right) .
\end{aligned}
$$

Letting $u_{\varepsilon}(x)$ be the first probability on the RHS of (5.42) we have that

$$
u_{\varepsilon}(x) \geq \int_{0}^{x} h_{\varepsilon}\left(x^{\prime}\right) d x^{\prime} / \int_{0}^{\sqrt{\delta}} h_{\varepsilon}\left(x^{\prime}\right) \exp \left[\frac{3}{2}\left(1+\frac{x^{\prime}}{\varepsilon}\right)^{2 / 3}\left(\frac{x^{\prime}}{L_{0}}\right)^{1 / 3}\right] d x^{\prime} .
$$

It is evident that the difference between the RHS of (5.37) and the RHS of (5.43) converges to zero as $\varepsilon \rightarrow 0$ provided $0<x<\delta^{2} / 2$. To estimate the second probability on the RHS of (5.42) we let $\tau_{x}$ be the time to exit $[0,2 \sqrt{\delta}]$ and put $v_{\varepsilon}(x)=E\left[\tau_{x}\right]$. Arguing as we did previously we have that

$$
\lim _{\varepsilon \rightarrow 0} v_{\varepsilon}(\sqrt{\delta})=\int_{\sqrt{\delta}}^{2 \sqrt{\delta}} \frac{d x}{1-\left(x / L_{0}\right)^{1 / 3}} .
$$

Similarly if we set $w_{\varepsilon}(x)=E\left[\tau_{x}^{2}\right]$ then

$$
\lim _{\varepsilon \rightarrow 0} w_{\varepsilon}(\sqrt{\delta})=\left[\int_{\sqrt{\delta}}^{2 \sqrt{\delta}} \frac{d x}{1-\left(x / L_{0}\right)^{1 / 3}}\right]^{2} .
$$

It follows from (5.44), (5.45) that the second probability on the RHS of (5.42) converges to 1 as $\varepsilon \rightarrow 0$. We conclude that the first term on the RHS of (5.34) is bounded by $4 \delta$ as $\varepsilon \rightarrow 0$, uniformly in $x$ for $0<x<\delta^{2} / 2$.

We turn to the second term on the RHS of (5.34). Letting $\tau_{x}$ be the exit time of $X_{\varepsilon}\left(s^{\prime}\right)$ with $X_{\varepsilon}(s)=x$ from the interval $[0,2 \sqrt{\delta}]$, then if we put

$$
v_{\varepsilon}(x)=E\left[\int_{s-\tau_{x}}^{s} \frac{d s^{\prime}}{X_{\varepsilon}\left(s^{\prime}\right)^{2 / 3}} \mid X_{\varepsilon}(s)=x\right],
$$

one has that $v_{\varepsilon}(x)$ satisfies

$$
\begin{gathered}
-\varepsilon(1+x / \varepsilon)^{1 / 3} v_{\varepsilon}^{\prime \prime}(x)-\left[1-\left(\frac{x}{L_{0}}\right)^{1 / 3}+\frac{2}{3}(1+x / \varepsilon)^{-2 / 3}\right] v_{\varepsilon}^{\prime}(x)=1 / x^{2 / 3}, \\
0<x<2 \sqrt{\delta}, v_{\varepsilon}(0)=v_{\varepsilon}(2 \sqrt{\delta})=0 .
\end{gathered}
$$

Hence $v_{\varepsilon}(x)$ is given in terms of the Dirichlet Green's function for (5.46) by

$$
v_{\varepsilon}(x)=\int_{0}^{2 \sqrt{\delta}} G_{\varepsilon}(x, y) / y^{2 / 3} d y
$$


Arguing as we did for the Green's function (5.39) we see that for $\delta$ sufficiently small $G_{\varepsilon}(x, y) \leq 2$, whence $v_{\varepsilon}(x) \leq 12 \delta^{1 / 6}$. To bound the second term on the RHS of (5.34) we need to add to it paths for which $\tau_{x}<\delta$ and $X_{\varepsilon}\left(\tau_{x}\right)=2 \sqrt{\delta}$. Such a path can eventually wander back to $x=\sqrt{\delta}$ and then we can estimate by $v_{\varepsilon}(\sqrt{\delta})$ the contribution to the integral of $X_{\varepsilon}\left(s^{\prime}\right)^{-2 / 3}$ until the path again hits $2 \sqrt{\delta}$. Thus the second term on the RHS of (5.34) is bounded by

$$
\begin{aligned}
\frac{1}{3 L_{0}^{1 / 3}}\left[v_{\varepsilon}(x)+v_{\varepsilon}\right. & (\sqrt{\delta})+P\left(\tau_{\sqrt{\delta}}<\delta\right) v_{\varepsilon}(\sqrt{\delta}) \\
& \left.+\left\{\sum_{k=2}^{\infty} P\left(\tau_{\sqrt{\delta}}<\delta\right)^{k}\right\} v_{\varepsilon}(\sqrt{\delta})+\delta /(\sqrt{\delta})^{2 / 3}\right] \leq K \delta^{1 / 6},
\end{aligned}
$$

for some constant $K$. Note the last term on the RHS of (5.46) is to take account of the integral of $X_{\varepsilon}\left(s^{\prime}\right)^{-2 / 3}$ when $X_{\varepsilon}\left(s^{\prime}\right)>\sqrt{\delta}$. We are also using (5.44), (5.45) to see that $P\left(\tau_{\sqrt{\delta}}<\delta\right)<1 / 2$ for small $\varepsilon$.

We consider the final term on the RHS of (5.34). Let $\tau_{x}$ be the time taken for the diffusion $X_{\varepsilon}\left(s^{\prime}\right), s^{\prime}<s$, with $X_{\varepsilon}(s)=x$ to exit the interval $[0,2 \sqrt{\delta}]$, and let $\tau_{x}^{\prime}$ be the corresponding time for $Y_{\varepsilon}\left(s^{\prime}\right), s^{\prime}<s$. Since $X_{\varepsilon}\left(s^{\prime}\right) \leq Y_{\varepsilon}\left(s^{\prime}\right), s^{\prime} \leq s$, it follows that for a path $X_{\varepsilon}\left(s^{\prime}\right), s^{\prime} \leq s$, with $X_{\varepsilon}\left(s-\tau_{x}\right)=2 \sqrt{\delta}$ then $\tau_{x}^{\prime}<\tau_{x}$. Hence the final term on the RHS of (5.34) is bounded by

$$
\begin{aligned}
& E\left[\frac{2}{9 \varepsilon} \int_{s-\tau_{x}}^{s}\left[1+X_{\varepsilon}\left(s^{\prime}\right) / \varepsilon\right]^{-5 / 3} d s^{\prime} ; \tau_{x}>\delta, X_{\varepsilon}\left(s-\tau_{x}\right)=0\right] \\
+ & E\left[\frac{2}{9 \varepsilon} \int_{s-\tau_{x}}^{s}\left[1+X_{\varepsilon}\left(s^{\prime}\right) / \varepsilon\right]^{-5 / 3} d s^{\prime} ; X_{\varepsilon}\left(s-\tau_{x}\right)=2 \sqrt{\delta}\right] \\
- & E\left[\frac{2}{9 \varepsilon} \int_{s-\tau_{x}^{\prime}}^{s}\left[1+Y_{\varepsilon}\left(s^{\prime}\right) / \varepsilon\right]^{-5 / 3} d s^{\prime} ; Y_{\varepsilon}\left(s-\tau_{x}^{\prime}\right)=2 \sqrt{\delta}\right] \\
+ & E\left[\frac{2}{9 \varepsilon} \int_{t}^{s}\left[1+X_{\varepsilon}\left(s^{\prime}\right) / \varepsilon\right]^{-5 / 3} d s^{\prime} ; \tau_{2 \sqrt{\delta}, s}<t \mid X_{\varepsilon}(s)=2 \sqrt{\delta}\right] .
\end{aligned}
$$

Letting $u_{\varepsilon}(x)$ be the first term in (5.48) we see that

$$
\begin{gathered}
-\varepsilon(1+x / \varepsilon)^{1 / 3} u_{\varepsilon}^{\prime \prime}(x)-\left[1-\left(\frac{x}{L_{0}}\right)^{1 / 3}+\frac{2}{3}(1+x / \varepsilon)^{-2 / 3}\right] u_{\varepsilon}^{\prime}(x) \\
=\frac{2}{9 \varepsilon}(1+x / \varepsilon)^{-5 / 3} P\left(\tau_{x}>\delta, X_{\varepsilon}\left(s-\tau_{x}\right)=0\right), \\
0<x<2 \sqrt{\delta}, u_{\varepsilon}(0)=u_{\varepsilon}(2 \sqrt{\delta})=0 .
\end{gathered}
$$

Next we put $v_{\varepsilon}(x)=E\left[\tau_{x} ; X_{\varepsilon}\left(s-\tau_{x}\right)=0\right]$. Then $v_{\varepsilon}(x)$ satisfies

$$
\begin{gathered}
-\varepsilon(1+x / \varepsilon)^{1 / 3} v_{\varepsilon}^{\prime \prime}(x)-\left[1-\left(\frac{x}{L_{0}}\right)^{1 / 3}+\frac{2}{3}(1+x / \varepsilon)^{-2 / 3}\right] v_{\varepsilon}^{\prime}(x)=P\left(X_{\varepsilon}\left(s-\tau_{x}\right)=0\right), \\
0<x<2 \sqrt{\delta}, v_{\varepsilon}(0)=v_{\varepsilon}(2 \sqrt{\delta})=0 .
\end{gathered}
$$

We can see from (5.37) that $P\left(X_{\varepsilon}\left(s-\tau_{x}\right)=0\right)<<1$ if $x>O(\varepsilon)$. Hence from our estimates on the Green's function (5.39) we conclude from (5.50) that $v_{\varepsilon}(x) \leq C \varepsilon$ 
for $0<x<2 \sqrt{\delta}$, where $C$ is a constant. Now using the Chebyshev inequality to bound the RHS of (5.49) we see that $u_{\varepsilon}(x) \leq C \varepsilon / \delta, 0<x<2 \sqrt{\delta}$.

Next we consider the second and third terms in (5.48). The sum of these two terms is given by $u_{\varepsilon}(x)-v_{\varepsilon}(x)$ where $u_{\varepsilon}(x)$ satisfies

$$
\begin{gathered}
-\varepsilon(1+x / \varepsilon)^{1 / 3} u_{\varepsilon}^{\prime \prime}(x)-\left[1-\left(\frac{x}{L_{0}}\right)^{1 / 3}+\frac{2}{3}(1+x / \varepsilon)^{-2 / 3}\right] u_{\varepsilon}^{\prime}(x) \\
=\frac{2}{9 \varepsilon}(1+x / \varepsilon)^{-5 / 3} P\left(X_{\varepsilon}\left(s-\tau_{x}\right)=2 \sqrt{\delta}\right), \\
0<x<2 \sqrt{\delta}, u_{\varepsilon}(0)=u_{\varepsilon}(2 \sqrt{\delta})=0,
\end{gathered}
$$

and $v_{\varepsilon}(x)$ satisfies

$$
\begin{aligned}
-\varepsilon(1+x / \varepsilon)^{1 / 3} v_{\varepsilon}^{\prime \prime}(x)-\left[1+\frac{2}{3}(1+x / \varepsilon)^{-2 / 3}\right] & v_{\varepsilon}^{\prime}(x) \\
=\frac{2}{9 \varepsilon}(1+x / \varepsilon)^{-5 / 3} P\left(Y_{\varepsilon}\left(s-\tau_{x}^{\prime}\right)\right. & =2 \sqrt{\delta}), \\
0 & <x<2 \sqrt{\delta}, v_{\varepsilon}(0)=v_{\varepsilon}(2 \sqrt{\delta})=0 .
\end{aligned}
$$

It is easy to see from (5.37) that

$$
\left|P\left(X_{\varepsilon}\left(s-\tau_{x}\right)=2 \sqrt{\delta}\right)-P\left(Y_{\varepsilon}\left(s-\tau_{x}\right)=2 \sqrt{\delta}\right)\right| \leq C \varepsilon^{1 / 3} .
$$

We may also easily estimate the difference in the Green's functions (5.39) for (5.51) and (5.52) to conclude that $\left|u_{\varepsilon}(x)-v_{\varepsilon}(x)\right| \leq C \varepsilon^{1 / 3}$ for $0<x<2 \sqrt{\delta}$.

We estimate the last term in (5.48) in a similar way to how we estimated the second term on the RHS of (5.34). We see that it is bounded by $\varepsilon^{2 / 3} / \delta^{1 / 3}$. We have therefore appropriately bounded the third term on the RHS of (5.34), whence the result follows.

Theorem 5.1. Let $c_{0}(x), c_{0}(x, t), c_{\varepsilon}(x, t)$ be as in Theorem 4.1 and $F(x, t)$ be the mapping defined after (4.1). If the function $c_{0}(\cdot)$ is continuous at $x=F(0, T)$ then (5.1) holds.

Proof. We use the fact that

$$
\frac{d}{d T} \int_{0}^{\infty} c_{\varepsilon}(x, T) d x=\int_{0}^{\infty} \rho_{\varepsilon}(x, 0, T) c_{0}(x) d x,
$$

where $\rho_{\varepsilon}$ is as in Lemma 5.3. We first show that

$$
\lim _{\varepsilon \rightarrow 0} \int_{0}^{\infty} \rho_{\varepsilon}(x, 0, T) c_{0}(x) d x=c_{0}(F(0, T)) \lim _{\varepsilon \rightarrow 0} \int_{0}^{\infty} \rho_{\varepsilon}(x, 0, T) d x .
$$

To do this we use the representation,

$$
\rho_{\varepsilon}(x, 0, T)=E\left[\rho\left(X_{\varepsilon}(t), t, T\right) ; \tau_{x, 0}>t \mid X_{\varepsilon}(0)=x\right],
$$

where $X_{\varepsilon}(s)$ is a solution to (4.7) with $X_{\varepsilon}(0)=x$ and $\tau_{x, 0}$ is the first hitting time at 0 . We choose now $t=T-c_{1} \varepsilon$ so we can bound $\rho_{\varepsilon}(\cdot, t, T)$ from Lemma 5.2. It follows from Lemma 5.2 that for $x>F(0, T)$ then

$$
\rho_{\varepsilon}(x, 0, T) \leq \frac{C}{\varepsilon}\left\{\exp \left[-(x / 8 \varepsilon)^{5 / 3}\right]+P\left(X_{\varepsilon}(t)<x / 2 ; \tau_{x, 0}>t\right)\right\} .
$$

It is evident that the first term on the RHS of (5.55) is small. We shall show that the second term is also small provided $x>>1+O(T)$. To do this let $\tau_{x}$ be the exit 
time for $X_{\varepsilon}(s)$ with $X_{\varepsilon}(0)=x$ from the interval $[x / 2,2 x]$ and define $Y_{\varepsilon}(s), s \geq 0$, by

$$
Y_{\varepsilon}(s)=\int_{0}^{s \wedge \tau_{x}} \sqrt{2 \varepsilon}\left(1+X_{\varepsilon}\left(s^{\prime}\right) / \varepsilon\right)^{1 / 6} d W\left(s^{\prime}\right) .
$$

Observe now that for any $\lambda, M_{\lambda, \varepsilon}(s)$ defined by

$$
M_{\lambda, \varepsilon}(s)=\exp \left[-\varepsilon \lambda^{2} \int_{0}^{s \wedge \tau_{x}}\left(1+X_{\varepsilon}\left(s^{\prime}\right) / \varepsilon\right)^{1 / 3} d s^{\prime}+\lambda Y_{\varepsilon}(s)\right], \quad s \geq 0,
$$

is a Martingale. Hence for any $a>0$ there is the inequality,

$$
\begin{aligned}
P\left(\sup _{0 \leq s \leq t} Y_{\varepsilon}(s)>a\right) & \leq P\left(\sup _{0 \leq s \leq t} M_{\lambda, \varepsilon}(s) \geq \exp \left[-\varepsilon \lambda^{2}(1+2 x / \varepsilon)^{1 / 3} t+\lambda a\right]\right) \\
& \leq \exp \left[\varepsilon \lambda^{2}(1+2 x / \varepsilon)^{1 / 3} t-\lambda a\right] .
\end{aligned}
$$

Minimizing the RHS of the last inequality w.r. to $\lambda$ we conclude that

$$
\left.P\left(\sup _{0 \leq s \leq t}\left|Y_{\varepsilon}(s)\right|>a\right) \leq 2 \exp \left[-a^{2} / 4 \varepsilon(1+2 x / \varepsilon)^{1 / 3}\right)\right] .
$$

Let $X_{\varepsilon}^{c}(s)$ be the classical solution to (4.7) with $X_{\varepsilon}^{c}(s)=x$, i.e. the solution to the deterministic equation when we eliminate the stochastic term. Then from Gronwall's inequality we have that

$$
\sup _{0 \leq s \leq t}\left|X_{\varepsilon}\left(s \wedge \tau_{x}\right)-X_{\varepsilon}^{c}\left(s \wedge \tau_{x}\right)\right| \leq \sup _{0 \leq s \leq t}\left|Y_{\varepsilon}(s)\right| \exp \left[C t / x^{2 / 3}\right],
$$

for some constant $C$. If we use the fact that $X_{\varepsilon}^{c}(s) \geq x-s$, we may conclude from the last two inequalities that

$P\left(X_{\varepsilon}(t)<x / 2 ; \tau_{x, 0}>t\right) \leq P\left(\tau_{x}<t\right) \leq 2 \exp \left[-x^{2} \exp \left(-C t^{1 / 3}\right) / 64 \varepsilon(1+2 x / \varepsilon)^{1 / 3}\right]$,

provided $x>4 t$. Thus we obtain an estimate on the RHS of (5.55) which falls off exponentially in $x / \varepsilon$, and we can easily extend this to all $x>F(0, T)+\delta$ for any $\delta>0$.

Next we consider the case $0<x<F(0, T)$, in which case we use the bound $\rho_{\varepsilon}(x, 0, T) \leq C P\left(\tau_{x, 0}>t \mid X_{\varepsilon}(0)=x\right) / \varepsilon$ for some constant $C$, which follows from (5.54) and Lemma 5.2. We use the comparison (4.10) and let $\tau_{x}$ be the exit time from the interval $[0, \delta]$ for the process $Y_{\varepsilon}(t)$ with $Y_{\varepsilon}(0)=x$. Then for a function $f(x)$, if we define $v_{\varepsilon}(x)$ by

$$
v_{\varepsilon}(x)=E\left[\exp \left\{\int_{0}^{\tau_{x}} f\left(Y_{\varepsilon}(t)\right) d t\right\} \mid Y_{\varepsilon}(0)=x\right],
$$

then $v_{\varepsilon}(x)$ satisfies

$$
\begin{gathered}
\varepsilon(1+x / \varepsilon)^{1 / 3} v_{\varepsilon}^{\prime \prime}(x)-\left[1-\left(x / L_{0}\right)^{1 / 3}\right] v_{\varepsilon}^{\prime}(x)+f(x) v_{\varepsilon}(x)=0, \\
0<x<\delta, v_{\varepsilon}(0)=v_{\varepsilon}(\delta)=1 .
\end{gathered}
$$

Observe now that the boundary value problem

$$
\eta u_{\eta}^{\prime \prime}(x)-\alpha u_{\eta}^{\prime}(x)+\lambda u_{\eta}(x)=0, \quad x>0, u_{\eta}(0)=1,
$$


has solution $u_{\eta}(x)$ given by the formula

$$
u_{\eta}(x)=\exp \left[\frac{\alpha-\sqrt{\alpha^{2}-4 \eta \lambda}}{2 \eta} x\right], \quad x>0,
$$

where we assume $\eta, \alpha, \lambda>0$ and $4 \eta \lambda<\alpha^{2}$. If $\eta \rightarrow 0$ then $u_{\eta}(x)$ converges to $\exp [\lambda x / \alpha]$, corresponding to the fact that the deterministic path starting at $x$ takes time $x / \alpha$ to hit 0 . It is clear that if we choose $\eta=\varepsilon(1+\delta / \varepsilon)^{1 / 3}$ and $\alpha=1-\left(\delta / L_{0}\right)^{1 / 3}$ then $v_{\varepsilon}(x)=u_{\eta}(x)$ satisfies (5.56) with $f(x) \geq \lambda$ and $v_{\varepsilon}(0)=1, v_{\varepsilon}(\delta)>1$. We conclude therefore that provided $4 \eta \lambda<\alpha^{2}$ there is the inequality,

$$
E\left[e^{\lambda \tau_{x}}\right] \leq u_{\eta}(x), \quad 0<x<\delta
$$

whence

$$
P\left(\tau_{x}>t\right) \leq e^{-\lambda t} u_{\eta}(x), t>0 .
$$

If we optimize now w.r. to $\lambda$ we conclude that $P\left(\tau_{x}>t\right)$ satisfies the inequality,

$$
P\left(\tau_{x}>t\right) \leq \exp \left[-(t \alpha-x)^{2} / 4 \eta t\right], \quad 0<x<\alpha t .
$$

It follows that if $0<x<\delta$ and $\delta$ is sufficiently small, depending on $T$, that $\lim _{\varepsilon \rightarrow 0} \rho_{\varepsilon}(x, 0, T)=0$, uniformly in $x$.

We can apply the arguments of the previous two paragraphs to show that for any $\delta>0$ then $\lim _{\varepsilon \rightarrow 0} \rho_{\varepsilon}(x, 0, T)=0$ uniformly for $x$ in the interval $0<x<F(0, T)-\delta$. In fact we have the inequality,

$$
\begin{array}{r}
P\left(\tau_{x, 0}>t \mid X_{\varepsilon}(0)=x\right) \leq P\left(\sup _{0 \leq s \leq t-\eta}\left|X_{\varepsilon}\left(s \wedge \tau_{x}\right)-X_{\varepsilon}^{c}\left(s \wedge \tau_{x}\right)\right|>\rho \delta / 2\right) \\
+\sup _{0 \leq y<(1+\rho) \delta / 2} P\left(\tau_{y}>\eta\right),
\end{array}
$$

where $\rho, \eta$ are small positive constants. The exit time $\tau_{y}$ in the second term on the RHS of (5.58) refers to the exit time from $[0, \delta]$ for $Y_{\varepsilon}(s)$ with $Y_{\varepsilon}(0)=y$. The exit time $\tau_{x}$ in the first term is the time taken for $X_{\varepsilon}(s)$ with $X_{\varepsilon}(0)=x$ to exit the interval $[\delta / 2, a]$, where $a=\sup \{x(s): 0 \leq s \leq T\}$ and $x(s)$ is the solution to (4.1) with $x(T)=0$. For the solution to 4.1) with $x(0)=x<F(0, T)-\delta$, let $\eta$ be defined by $x(T-\eta)=(1-\rho) \delta / 2$ for some small $\rho>0$ to be determined. Then $X_{\varepsilon}^{c}(t-\eta) \leq \delta / 2$ for small $\varepsilon$, whence (5.58) holds for sufficiently small $\varepsilon$. We can choose now $\rho>0$ small enough so that $\eta \geq(1+2 \rho) \delta / 2$. In that case both terms on the RHS of (5.58) go to zero exponentially in $1 / \varepsilon^{2 / 3}$ as $\varepsilon \rightarrow 0$. We have therefore proved (5.53).

We complete the proof of the theorem by showing that

$$
\lim _{\varepsilon \rightarrow 0} \int_{0}^{\infty} \rho_{\varepsilon}(x, 0, T) d x=\exp \left[-\frac{1}{3} \int_{0}^{T} \frac{d s}{x(s)^{2 / 3} L(s)^{1 / 3}}\right],
$$

where $x(s)$ is the solution to (4.1) with $x(T)=0$. To do this we write as in (5.20)

$$
\int_{0}^{\infty} \rho_{\varepsilon}(x, 0, T) d x=\int_{0}^{\infty} \rho_{\varepsilon}(x, T-\delta, T) c_{\varepsilon}(x, T-\delta) d x .
$$


Since by our previous argument $\rho_{\varepsilon}(x, T-\delta, T)$ is concentrated at $x \sim \delta$, (5.59) will follow from Lemma 5.3 if we can show that

$$
\lim _{\varepsilon \rightarrow 0} c_{\varepsilon}(x, T-\delta)=\exp \left[-\frac{1}{3} \int_{0}^{T-\delta} \frac{d s}{x(s)^{1 / 3} L(s)^{1 / 3}}\right],
$$

where $x(s)$ is the solution to (4.1) with $x(T-\delta)=x \sim \delta$. This follows as before using the representations (5.21), (5.22) for $c_{\varepsilon}(x, s)$ and comparing solutions of the stochastic equation to solutions of the corresponding deterministic equation.

\section{Discussion}

This paper is primarily concerned with the study of a diffusive version of the LSW model and the convergence of its solutions on any finite time interval as the coefficient of diffusion $\varepsilon$ goes to 0 to solutions of the classical LSW model. This is a first step towards showing that solutions of the diffusive LSW equation behave similarly to solutions of the LSW equation for arbitrarily large time. In particular, one expects that solutions of the diffusive LSW equation coarsen in a fashion similar to solutions of LSW. Thus the average cluster volume is expected to increase linearly in time at large times, and this should be uniform in $\varepsilon$ for small $\varepsilon$. In Theorem 3.2 we obtained a uniform time averaged upper bound on the rate of coarsening.

Although the effect of the diffusion decreases with time, discussion in the physics literature suggests that it plays an important role in the asymptotic behavior of the solution by acting as a selection principle. Thus at moderate times the diffusion produces a Gaussian tail to initial data (with compact support for example). It is the tail which determines asymptotic behavior, singling out the infinitely differentiable self-similar solution of the LSW model.

The paper begins with a derivation of the diffusive LSW model from the BeckerDöring model. The least justified step in the derivation is the imposition of a Dirichlet boundary condition, which cuts off interaction between clusters of large volume and clusters of $O(1)$ volume that are almost in equilibrium at large time. The boundary condition is imposed at a particular cluster volume. One can see that this is a significant simplification since it implies that after the condition is imposed, the monomer density is for all subsequent times strictly larger than its equilibrium value. Despite this issue, the derivation of the diffusive LSW model from the BD model does offer a strategy for attempting to understand the mechanism of coarsening in the BD model. In order to carry it through one will have to understand in a precise way how a boundary condition is imposed on the diffusive LSW model by the BD dynamics.

There has been some previous literature showing in an almost mathematically rigorous way a connection between solutions of the LSW and BD models. The key assumption required in this work is a type of upper bound on the coarsening rate for the BD model. It is not clear how this is related to the boundary condition assumption discussed in the previous paragraph. One should note however that a positive lower bound on the monomer density minus its equilibrium value, which is a consequence of the Dirichlet boundary condition, is a type of upper bound on the coarsening rate. 
Acknowledgement: The author would like to thank Peter Smereka and Barbara Niethammer for many helpful conversations. This research was partially supported by NSF under grants DMS-0500608 and DMS-0553487.

\section{REFERENCES}

[1] Ball, J.M.; Carr, J.; Penrose, O. The Becker Döring cluster equations: basic properties and asymptotic behavior of solutions. Comm. Math. Phys. 104 (1986), 657-692, MR 0841675.

[2] Becker, R.; Döring, W. Kinetische Behandlung der Keimbildung in übersättigten Dämpfen. Ann. Phys. (Leipzig) 24 (1935), 719-752.

[3] Collet, Jean-Francois; Goudon, Thierry. On solutions of the Lifschitz-Slyozov model. Nonlinearity 13 (2000), 1239-1262. MR 1767957.

[4] Dai, Shibin; Pego, Robert L. Universal bounds on coarsening rates for mean-field models of phase transitions. SIAM J. Math. Anal. 37 (2005), 347-371, MR 2176107.

[5] Farjoun, Y.; Neu, John C. Exhaustion of nucleation in a closed system. Physical Review E $\mathbf{7 8}$ (2008), 051402 (7 pages).

[6] Freidlin, M.I.; Wentzell, A. D. Random perturbations of dynamical systems. Second Edition. Springer-Verlag 1998. 430 pp. MR 1652127.

[7] Karatzas, I.; Shreve, S. "Brownian Motion and Stochastic Calculus", second edition. Graduate Texts in Mathematics 113. Springer-Verlag, New York, 1991. MR 1121940.

[8] Kohn, Robert V.; Otto, Felix. Upper bounds on coarsening rates. Comm. Math. Phys. 229 (2002), 375-395, MR 1924360.

[9] Laurençot, Philippe. The Lifshitz-Slyozov-Wagner equation with conserved total volume. SIAM J. Math. Anal. 34 (2002), 257-272. MR 1951774.

[10] Laurençot, Philippe; Mischler, Stéphanie. From the Becker-Döring to the Lifshitz-SlyozovWagner Equations. J. Statist. Phys. 106 (2002), 957-991. MR 1889598.

[11] Lifschitz, I. M.; Slyozov, V. V. Kinetics of precipitation from supersaturated solid solutions. J. Phys. Chem. Sol. 19 (1961), 35-50.

[12] Meerson, B. Fluctuations provide strong selection in Ostwald ripening. Physical Review E 60 (1999), 3072-3075.

[13] Niethammer, B. On the evolution of large clusters in the Becker Döring model. J. Nonlinear Sci. 13 (2003), 115-155. MR 1950848.

[14] Niethammer, B. ; Pego, Robert L. Non-self-similar behavior in the LSW theory of Ostwald ripening. J. Statist. Phys. 95 (1999), 867-902, MR 1712441.

[15] Niethammer, B. ; Pego, Robert L. On the initial value problem in the Lifschitz-SlyozovWagner theory of Ostwald ripening. SIAM J. Math. Anal. 31 (2000), 467-485, MR 1735959.

[16] Niethammer, B. ; Pego, Robert L. Well-posedness for measure transport in a family of nonlocal domain coarsening models. Indiana Univ. Math. J. 54 (2005), 499-530, MR 2136819.

[17] Niethammer, B. ; Velázquez, J.J.L. Global stability and bounds for coarsening rates within the mean-field theory for domain coarsening. Comm. Partial Differential Equations 31 (2006), 1679-1708, MR 2273970.

[18] Niethammer, B. ; Velasquez, J.J.L. On the convergence to the smooth self-similar solution in the LSW model. Indiana Univ. Math. J. 55 (2006), 761-794, MR 2225452.

[19] Pego, Robert L. "Lectures on dynamics in models of coarsening and coagulation", in Dynamics in models of coarsening, coagulation, condensation and quantization, 1-61, Lecture Notes Ser. Inst. Math. Sci. Natl. Univ. Singap., 9, World Sci. Publ., Hackensack, NJ, 2007. MR 2395779.

[20] Penrose, O. The Becker Döring equations at large times and their connection with the LSW theory of coarsening. J. Statist. Phys. 89 (1997), 305-320, MR 1492493.

[21] Velázquez, J.J.L. The Becker-Döring Equations and the Lifshitz-Slyozov-Wagner Theory of Coarsening. J. Statist. Phys. 92 (1998), 195-236. MR 1645647.

[22] Wagner, C. Theorie der alterung von niederschlägen durch umlösen. Z. Elektrochem. 65 (1961), 581-591.

University of Michigan, Department of Mathematics, Ann Arbor, Mi 48109-1109

E-mail address: conlon@umich.edu 